\title{
Active Translation Control of CD4 T Cell Activation by Regulatory T Cells
}

\author{
Lomon $\mathrm{So}^{1,3}$ Kazushige Obata-Ninomiya ${ }^{1}$, Alex $\mathrm{Hu}^{2}$, Virginia Muir ${ }^{2}$, Ayako Takamori ${ }^{1}$ \\ Ram Savan ${ }^{3 *}$, and Steven F. Ziegler ${ }^{1,3^{*}}$ \\ Centers for Fundamental ${ }^{1}$ and Systems Immunology ${ }^{2}$, Benaroya Research Institute, Seattle, WA USA \\ ${ }^{3}$ Department of Immunology, School of Medicine, University of Washington, \\ Seattle, WA 98109 USA.
}

*Correspondence addressed to: savanram@uw.edu and sziegler@benaroyaresearch.org

\begin{abstract}
SUMMARY
Increased protein synthesis is a hallmark of lymphocyte activation. Regulatory T cells (Tregs) suppress the activation and subsequent effector functions of CD4 effector T cells (Teff). Molecular mechanisms that enforce suppression on CD4 Teff cell function are unclear. Control of CD4 Teff cell activation by Tregs has largely been defined at the transcriptional level, which does not reflect changes in posttranscriptional control. We found that Tregs suppressed activation-induced global protein synthesis in CD4 Teff cells prior to cell division. We analyzed genome-wide changes in the transcriptome and translatome of activated CD4 Teff cells using two independent approaches. We show that mRNAs encoding for the protein synthesis machinery are regulated at the level of translation in activated Teff cells. Strikingly, Tregs suppressed global protein synthesis of CD4 Teff cells by specifically inhibiting mRNAs of the translation machinery at the level of mTORC1-mediated translation control. Lastly, we found that the RNA helicase eIF4A inhibitor rocaglamide A (RocA) can suppress CD4 Teff activation in vitro to alleviate inflammatory CD4 Teff activation caused by acute Treg depletion in vivo. These data provide evidence that peripheral tolerance is enforced by Tregs, mediated by IL-10, through mRNA translational control in CD4 Teff cells. Therefore, therapeutic targeting of the protein synthesis machinery can mitigate inflammatory responses invoked by Treg loss of function.
\end{abstract}




\section{INTRODUCTION}

Most self-reactive $\mathrm{T}$ cells are eliminated in the thymus through the process of central tolerance. However, a small percentage of cells escape to the periphery, where they have the potential to promote autoimmunity. These cells are normally held in check by a population of CD4 T cells referred to as regulatory T cells (Tregs). Tregs are essential to maintain immune homeostasis, and the transcription factor FOXP3 has been shown to be central to the development and function of Tregs. Mutations in FOXP3 gene in mice and human patients with IPEX syndrome develop a common set of autoimmune symptoms(Bennett et al., 2001; Ochs et al., 2007; Patel, 2001; Wildin et al., 2001). Mutations in the FOXP3 gene and the autoimmune phenotype is linked to a loss of Tregs or their function(Bacchetta et al., 2018; Ochs et al., 2007). Tregs have the ability to potently suppress CD4 effector T cells (Teff) either directly or through the modulation of antigen presenting cells (mainly DCs) to ultimately suppress activation, proliferation, and subsequent effector functions of Teff cells(Josefowicz et al., 2012; Tang and Bluestone, 2008; Vignali et al., 2008). Several mechanisms have been proposed for Treg-mediated suppression, including release of suppressive cytokines (e.g., TGF $\beta$, IL-10, IL-35) and expression of inhibitory receptors (e.g., CTLA-4, PD-1, TIGIT)(Shevach, 2009; Sojka et al., 2008). Although a block in proliferation and effector $\mathrm{T}$ cell function have been the hallmarks of Treg mediated suppression, the molecular changes in target Teff cells following Treg encounter still remains unclear. This is especially true for the first 24 hours prior to the onset of proliferation, when the biosynthetic capacity of the cell is greatly expanded(So et al., 2016; Wang et al., 2011).

Upon activation, resting $\mathrm{T}$ cells undergo a rapid biosynthetic and metabolic reprogramming in preparation for cell division(Manfrini et al., 2020; Ricciardi et al., 2018; Wang et al., 2011; Wolf et al., 2020). Included in this reprogramming is an increase in translational activity and capacity(Araki et al., 2017; Bjur et al., 2013). In this study, we show that Tregs suppress activation of Teffs by enforcing a global inhibition of mRNA translation. We assessed the genome-wide changes in transcriptome and translatome in activated CD4 Teff cells and identified translation control of mRNAs encoding components of the protein synthesis machinery. In the first 24 hours of Teff cell activation a set of mRNAs encoding proteins involved in the translational machinery, and whose transcription was unchanged, are shifted to polysomes. We found that Tregs specifically inhibit the shift of these mRNAs to polysomes by suppressing mammalian target of rapamycin complex 1 (mTORC1) signaling. In support of these findings, we provide new evidence that direct targeting of protein synthesis using rocaglamide A (RocA), a RNA helicase eIF4A inhibitor, inflammatory CD4 Teff activation caused by in vivo Treg loss can be alleviated. In summary, we provide a novel mechanism of Treg-mediated suppression of CD4 Teff activation through inhibition of protein synthesis machinery mRNAs at the post-transcriptional level and that such biological mechanism can be therapeutically targeted using small molecule inhibitors.

\section{RESULTS}

\section{Tregs control protein synthetic capacity of activated CD4 Teff cells.}

The 24-48 hours following CD4 Teff cell activation are critical for subsequent proliferation and expansion. This is a period where cellular biomass is accumulated through expansion of global protein synthetic capacity in preparation for cell division. We reasoned that this time period would be a target for Treg-mediated suppression in order to inhibit cell activation prior to proliferation. Overall protein 
synthesis rate can be quantified at the single-cell level by pulsing cells with the tRNA-analog puromycin (PMY) and intracellular staining for PMY. We co-cultured CD4 Tconv cells (CD4 $\left.{ }^{+} \mathrm{CD} 25^{-}\right)$and congenically marked Tregs (CD4+Foxp3+) at varying ratios with anti-CD3/CD28 coated beads and pulsed the culture with PMY. CD4 Tconv cells co-cultured with Tregs exhibited marked inhibition of proliferation in a dose-dependent manner (Figure S1A). Interestingly, Tconv cells co-cultured with Tregs clearly showed significantly less PMY incorporation in a Treg-dose-dependent manner before the onset of proliferation (Figure 1A). The pattern of suppression was not bimodal indicating that protein synthesis rate of all responding Tconv cells were modulated by Tregs but not completely compared to cycloheximide (CHX) treatment (Figure 1B). Since anti-CD3/CD28 beads were used to activate both populations of cells in the co-culture system, the downregulation of protein synthesis by Tregs is independent of antigen-presenting cells (APC). The Treg-mediated translational inhibition was observed as early as 6 hours post-activation, well before any metabolic changes occur in T cells (Figure 1C). Suppression was primarily due to early prevention of $\mathrm{T}$ cell activation as we found no difference in the ability of CD4 Teff cells to downregulate CD62L with or without Tregs (Figure S1B). Thus, suppression of global protein synthesis in CD4 Teff cells by Tregs could not be attributed to dampening or cold inhibition of general $\mathrm{T}$ cell activation. To test if similar regulatory pathways were operative in human $\mathrm{T}$ cells, we established ex vivo Treg suppression assays using PMY incorporation as the readout. We used in vitro-expanded Tregs from a single donor, and Tconv (defined as CD4+CD45RA+CD127+CD25-) from 5 individual healthy donors. We found that 24 hours of stimulation resulted in a significant increase in PMY staining, both in percentage of cells labeled and the MFI of PMY staining. In the cultures containing Tregs PMY incorporation was significantly reduced, both in total incorporation and in the percentage of cells that incorporated PMY (Figure 1D). These data demonstrate that Treg-mediated inhibition of activation-induced translation is a conserved function.

Next, to assess the role of Tregs in controlling protein synthesis in CD4 T cells in vivo, we acutely depleted Tregs through diphtheria toxin (DT) treatment of Foxp $3^{\text {DTR }}$ mice(Kim et al., 2007). Within 3 days post initial DT-induced depletion of Tregs (2 consecutive DT injections on day 0 and 1), we observed rapid appearance of a CD4 T cell population with significantly elevated incorporation of puromycin ex vivo compared to the PBS treated control mice, suggesting activation of the autoreactive CD4 T cell pool in the periphery (Figure 1E). When CD4 T cells from spleen and lymph nodes of DTtreated Foxp $3^{D T R}$ mice were purified and stimulated ex vivo, they proliferated with faster kinetics and were significantly larger in size indicating an aberrantly enhanced protein synthesis capacity in Teff cells activated in vivo attributed to Treg loss (Figure S1C). These data suggest that Tregs are both sufficient and necessary to suppress the rapid upregulation of protein synthesis in activated CD4 T cells both in vitro and in vivo.

To uncover the underlying mechanism of Treg-mediated translational inhibition we examined signaling pathways downstream of TCR stimulation in activated CD4 T cells. Specifically, we examined the mammalian target of rapamycin (mTOR) signaling pathway as it has been shown to be critical for coordinating cell growth and proliferation in lymphocytes through the eukaryotic translation initiation factor 4E (eIF4E) in translation initiation(Wardman et al., 2016). mTOR exists in 2 multi-protein complexes, mTOR complex 1 (mTORC1) and mTOR complex 2 (mTORC2)(Jhanwar-Uniyal et al., 2019; Zoncu et al., 2011). To assess mTORC1 signaling, we examined phosphorylation of ribosomal protein S6 (rpS6: S240/244) and eIF4E-binding proteins (p4EBP1/2: T37/46) at their respective 
mTORC1 specific phosphosites in activated CD4 T cells. mTORC2 signaling was assessed by phosphorylation of AKT at S473. As expected, both mTORC1 and mTORC2 signaling increased in activated CD4 T cells. Strikingly, Tregs significantly suppressed mTORC1 signaling (rpS6 and p4EBP1/2). However, mTORC2 signaling (AKT S473) remained intact, as did the PDK1- and PI3Kdependent phosphosite T308 which is more proximal to TCR engagement (Figure 1F). Using Nur77-GFP reporter Teffector cells, we see no differences in Teff cells co-cultured with Tregs indicating no change in proximal TCR signaling (Figure S1D). These findings are consistent with previous data showing that genetic or chemical inhibition of mTORC1 significantly inhibits lymphocyte proliferation in a 4EBP/eIF4E- dependent manner to control translation initiation in various cell types including lymphocytes (So et al., 2016; Thoreen et al., 2012).

\section{Translatome capture by RiboTag and novel method called Simple Polysome Efficient Extraction and Distribution (SPEED).}

Based on our observation that Tregs suppress protein synthesis in Teffs, we investigated if Tregs affect global mRNA translation using genome-wide approaches to interrogate changes in both the transcriptome and translatome of CD4 Tconv cells. First, we used the RiboTag system to capture translatome changes in $\mathrm{CD} 4 \mathrm{~T}$ cells. This system utilizes an HA epitope-tagged ribosomal protein large subunit L22 (eL22) that allows for immunoprecipitation of proteins and mRNA associated with ribosomes (Ribo-IP)(Sanz et al., 2009). RiboTag CD4 T cells were generated using Cd4-Cre mice (eL22 ${ }^{\text {Ribofff }}$ Cd4Cre: T-Ribo) (Figure S4A-B).

While the data using the Ribo-Tag system showed that Tregs can control mRNA-ribosome association in Teff cells, it does not distinguish between mRNAs with low ribosome occupancy (monosomes) from those associated with polysomes (Supplement text 1, Figure S4). The transition of mRNAs from monosome to polysome and back is a critical aspect of translation control. To resolve and distinguish mRNAs bound to polysomes from monosome-associated mRNAs we optimized the classical polysome profiling approach for low input cytosolic lysates (Figure 3A). We reasoned that by assessing the quantity and quality of total RNA extracted from fraction, we could determine ribosome positions since total RNA is mainly composed of ribosomal RNA (rRNA). Using lysates prepared from as few as 500,000 to 1 million activated CD4 T cells, we found that total RNA extracted from each fraction and plotted as a percent distribution plot closely resembled a classical A254nm polysome trace obtained using

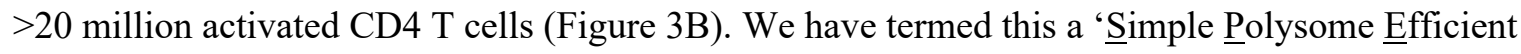
Extraction and Distribution' (SPEED) plot to distinguish it from the traditional polysome traces requiring greater cellular input (Figure 3C). Furthermore, qualitative analysis of the extracted RNA using Bioanalyzer gave us information as to the position of the intact $80 \mathrm{~S}$ monosome in the gradient (Figure 3C). The SPEED plots generated from unstimulated CD4 T cells showed most of the total RNA to be enriched in the monosome fraction (Figure 3C), confirming our observations that CD4 T cells prior to activation have low translational activity (Figure 1A). Upon stimulation, nearly 50\% of the monosomes shifted towards the heavier sucrose fractions indicating the assembly of polysomes and increased protein synthesis (Figure 3C). To ensure SPEED plots were a faithful representation of the mRNA translational status of a cell, we took advantage of the translation initiation inhibitor harringtonine, which only interferes with initiating ribosomes and allows elongating ribosomes to run-off(Fresno et al., 1977). Activated CD4 Teff cells were treated with homoharringtonine (HHT) for $10 \mathrm{~min}$ to allow run-off elongation of ribosomes before cytosolic lysate preparation. Remarkably, SPEED plot from HHT treated activated CD4 Teff cells resembled unstimulated CD4 T cells with majority of ribosomes enriched in the 
monosome fraction (Figure 3C). Lastly, the distribution of beta-actin (Actb) mRNA was analyzed using quantitative PCR (qPCR) from each fraction. We chose Actb as it is routinely used as a housekeeping control mRNA as it has no apparent cis-regulatory sequence motif in its 5' untranslated region (UTR) and is highly translated. Despite low polysome levels in unstimulated CD4 Teff cells, we found Actb mRNA to be abundantly enriched in the polysome fractions. Activated CD4 Teff cells also translated Actb mRNA with high efficiency. As expected for a highly translated mRNA, HHT treatment led to a complete shift in $A c t b$ mRNA towards the lighter sucrose fractions indicating successful ribosome run-off (Figure 3D). In summary, our SPEED technique faithfully captured the translational status of cellular lysates from low biological input, making it ideal to assess translatome of primary immune cells and most importantly, in Treg suppressed CD4 Teff cells.

To determine the identity of the mRNAs that shift between monosomes and polysomes following activation we performed SPEED on CD4 T cells activated for $24 \mathrm{~h}$ with CD3/CD28 beads. A portion $(10 \%)$ of cellular lysate was used to isolate total RNA, while the remainder was subjected to SPEED analysis with monosome and polysome fractions collected and RNA isolated and sequenced (Figures 4A, S4 and S5). We found approximately 380 genes whose overall RNA level was unchanged but whose mRNAs were shifted from monosomes to polysomes following activation (Figures 3B and C). Interestingly, gene ontology analysis of these genes showed that the vast majority fell into functional categories involving ribosome biogenesis and mRNA translation, consistent with preparation for subsequent cell division following activation (Figures 3D and E). It is important to note that the genes that encode these mRNAs are considered to be housekeeping genes whose expression is very high and largely unchanged by cell activation. The data presented here demonstrates that while the RNA level of transcripts encoding the transcriptional machinery is unchanged, their translation is likely increased following activation due to their shift onto polyribosomes.

\section{Treg sensitive mRNAs are enriched for terminal oligopyrimidine (TOP) motif.}

We next determined the fate of these mRNAs in Teff cells stimulated in the presence of Tregs. We examined the mRNAs that showed increased translation efficiency (TE) in stimulated vs. resting cells and calculated their TE in CD4 T cells stimulated in the presence of Tregs (Stim+Treg). The set of mRNAs showing increased TE in stimulated cells were specifically reduced in their TE when the cells were stimulated in the presence of Tregs (Figure 3C). These data are consistent with our hypothesis that Treg-mediated translational control targets mRNAs involved in preparing the cell for subsequent proliferation, with ribosome biogenesis and translational activity being critically important for this process. The finding that stimulation in the presence of Tregs reversed the positive TE changes in transcripts encoding proteins involved in mRNA translation is consistent with data showing that overall protein synthesis is suppressed by Tregs and our observations using the RiboTag system (Figures 1 and 2). To identify the mechanism(s) that underlie Treg-specific translation control, we first examined cisregulatory elements shared by the 5'UTRs among Treg-sensitive mRNAs identified by SPEED (Supp Table 1). We searched for novel sequence-specific motifs within target UTRs in these Treg sensitive genes by retrieving murine 5'UTR sequences from Ensembl BioMart database (Ensembl Genes78, Mus musculus) and using Multiple Em for Motif Elicitation (MEME), to uncover common cis-regulatory elements(Cunningham et al., 2014; Leppek et al., 2018; Truitt et al., 2015). We found an oligopyrimidine tract enriched in the 5'UTR in Treg sensitive mRNAs (Figure 4A). The terminal oligopyrimidine (TOP) motif is a well-characterized motif which regulates key mRNAs encoding the translational machinery. 
The TOP motif is known to be present in the 5'UTR of ribosomal proteins (RPs) and other genes required for mRNA translation(Meyuhas et al., 1987). Interestingly, TOP motif containing RP transcripts are regulated by the well-known signaling kinase mammalian target of rapamycin (mTORC1)(Schneider et al., 2013; Thoreen et al., 2012). We further validated select genes that were differentially regulated in SPEED RNA-seq. We observed Rps10, Rpl14, Dhx34, eIF3e, and Rpl8, mRNAs shift to polysomes upon CD4 $\mathrm{T}$ cell stimulation and back to monosomes following activation in the presence of Tregs (Figure 4B). This shift does not reflect a change in the RNA level of these genes, but rather is a change in the translation of these mRNAs. As predicted, the mRNA distribution shifted back to the monosome fraction when the CD4 T cells were stimulated in the presence of Tregs. As a control, ActB mRNA is unchanged by Tregs. These data confirm global changes in the translatome induced by Tregs to control CD4 T cell activation by blocking the translation of a subset of mRNAs, thereby blunting the ability of these cells to respond appropriately to stimulation.

\section{IL-10 from Tregs block protein synthesis in Teffector cells}

Since the activation of CD4 T cells in the presence of Tregs resulted in a specific inhibition of mTORC1 activation, as indicated by the lack of ribosomal protein S6 and 4E-BP1/2 phosphorylation, we hypothesized that Tregs suppress the immediate biosynthetic response to antigen-specific activation through regulation of mTORC1-mediated translational control. To understand the pathways leading to the inhibition of mTORC1 activation in stimulated CD4 T cells by Tregs, we examined the role of soluble factors known to be produced by Tregs, using recombinant proteins and neutralizing antibodies specific for anti-inflammatory factors. While blocking IL-2 mediated signaling in stimulated T cells with IL-2 or CD25 antibodies showed a slight reduction in pS6, we did not observe significant effects on 4E-BP1 or puromycin incorporation, a surrogate of protein synthesis (Figure 5A). Treatment with recombinant TGF$\beta$ failed to suppress the mTORC1 pathway or protein synthesis (Figure 5B). However, treatment with recombinant IL-10 significantly reduced pS6, p4E-BP1, and puromycin incorporation in stimulated CD4 T cells (Figure 5C). Furthermore, we also observed inhibition of Treg mediated suppression of mTORC1 signaling and protein synthesis by $\alpha$ IL10 antibody treatment (Figure 5D). We saw a similar effect when Tregs were cocultured with stimulated IL-10 $\mathrm{Rb}^{-/-} \mathrm{CD} 4 \mathrm{~T}$ cells (Figure 5E). These data suggest that Tregs use IL-10 to disrupt mTORC1 signaling, affecting mRNA translation in stimulated CD4 T cells.

\section{Acute inflammation due to Treg deficiency rescued by an mRNA translation inhibitor}

While we show that mRNA translation suppression by Tregs has a direct effect on cell activation and proliferation, we wanted to investigate if this is an important mechanism by which Tregs exert their function downstream of mTORC1. To test this, we used a translation inhibitor Rocaglamide A (RocA, $1 H-2,3,3 \mathrm{a}, 8 \mathrm{~b}$-tetrahyrocyclopenta[ $b]$ benzofuran), which has been shown to reduce overall protein synthesis but exhibit preferential inhibition of a specific subset of mRNAs in cell culture models(Iwasaki et al., 2019). RocA is a secondary metabolite from the plant genus Aglaia with anti-tumor and antiinflammatory properties (Ebada et al., 2011; Li-Weber, 2015) (Bordeleau et al., 2008; Cencic et al., 2009; Li-Weber, 2015). RocA affects protein synthesis by binding to eIF4A, a DEAD-box RNA helicase, that is part of the eIF4F mRNA initiation complex (Rogers et al., 2002). RocA binds to eIF4A and clamps it on poly-purine sequences in the 5' untranslated regions of mRNAs, thereby preferentially inhibiting these set of mRNAs at the level of translation control where stable RocA-eIF4A-mRNA complexes are formed to block 43S pre-initiation complex scanning (Ernst et al., 2020; Iwasaki et al., 2019). While RocA has been shown to inhibit overall protein synthesis through the translational blockade of a subset of mRNAs 
(Iwasaki et al., 2016), its effect on CD4 T cell activation and proliferation is unclear. To address this, we measured protein synthesis rate in activated CD4 $\mathrm{T}$ cells $24 \mathrm{~h}$ post RocA treatment using the puromycylation assay (Schmidt et al., 2009; Seedhom et al., 2016). RocA-treated cells showed a dosedependent decrease in puromycin incorporation, suggesting inhibition of protein synthesis. We also found that RocA treatment inhibited cell-proliferation in a dose-dependent manner at sub nanomolar concentrations (Figures 6A, B). Surprisingly, early T cell activation genes such as IL-2 and CD25 (IL$2 \mathrm{R} \alpha$ ) were unaffected both at the mRNA and protein level by RocA, suggesting that early TCR signaling dependent on NFAT is not how RocA inhibits T cell proliferation. Furthermore, this supports the notion that translational blockade through RocA impacts subset of mRNAs even in T cells. As expected, when cells were treated with the calcineurin inhibitor Tacrolimus (FK506), T cell proliferation was inhibited with corresponding downregulation of both IL-2 and CD25 (IL-2R $\alpha$ ) (Figure 6C). These results suggest that while both RocA and FK506 inhibit CD4 T cell activation and proliferation, they do so through a different mechanism. While our finding is different from a previous study suggesting that RocA inhibits NFAT activity in human CD4 T cells (Proksch et al., 2005), we propose at higher concentrations (50-100 fold) RocA could affect transcription regulation.

\section{Inflammation mediated by Treg deficiency rescued by an mRNA translation inhibitor.}

The data presented above demonstrated that RocA-mediated translation inhibition could suppress CD4 $\mathrm{T}$ cell proliferation ex vivo. To extend these studies we determined whether RocA administration could ameliorate inflammation-induced CD4 T cell activation in vivo. First, we assessed the effect of RocA on the general health of the mice and immune homeostasis by treating mice every other day for a week with RocA (0.5 and 1mg/kg i.p.) (Figure S6). We found no differences in the overall weight with RocA treatment, nor in various cell populations within the CD $45^{+}$compartment, including CD4+ T cell subsets indicating no deleterious effects on immune homeostasis (Figure S6). Thus, short-term in vivo treatment with RocA was well tolerated by the mice as we did not observe any significant changes in the general health of these animals.

We next asked whether inflammatory responses were limited by RocA treatment in the absence of Tregs. For these studies we used the Foxp $3^{\text {DTR }}$ mice, treated with DT, to deplete Tregs as described in Figure 1D. To test the ability of RocA to affect acute inflammation we dosed Foxp $3^{\text {DTR }}$ mice with RocA $(0.2 \mathrm{mg} / \mathrm{kg}$ i.p.) or DMSO (vehicle) on days -1 and -2 , and then treated with DT (400 ng i.p.) on days 0,2 , 4 , and 6 , followed by sacrifice and analysis on day 8 (Figure 6E). A hallmark feature of Treg depletion is a rapid loss of body weight, which is seen in the vehicle-treated mice on day 7 with an average weight loss of $20 \%$ of initial body weight (Figure 6F). We found that the RocA-treated mice showed significantly less weight loss, averaging between $85-90 \%$ of starting weight, suggesting that RocA treatment was blunting the inflammation caused by Treg depletion. Consistent with this we found that numbers of splenic CD4 T cells producing IL-17A, IFN $\gamma$, and both were significantly reduced in RocAtreated mice (Figure 6G). Especially important was the reduction in IFN $\gamma / \mathrm{IL}-17 \mathrm{~A}$ co-producers as this subset has been found to be pathogenic in inflammatory settings (Doodes et al., 2010; Duhen et al., 2013; Kaiser et al., 2016; Lee et al., 2009). These data demonstrate that RocA-mediated direct translation inhibition can ameliorate the inflammation seen in mice lacking functional Tregs, supporting our findings that Treg-mediated immune tolerance on CD4 T cells is through an active translation control on the subset of mRNAs required for protein synthesis itself. 
Lastly, we also found suppression of proliferation in primary human T cells treated with RocA and stimulated with $\alpha \mathrm{CD} 3 / \mathrm{CD} 28$ (Figure $6 \mathrm{H}$ ). Similar to what was seen in mouse CD4 T cells, there was no difference in the surface expression of CD25 (Figure $6 \mathrm{H}$ ). We also observed significant suppression of IFN $\gamma$ producing cells with RocA treatment compared to the vehicle control post PMA/ionomycin stimulation (Figure 6I). Altogether, here we show a novel mechanism of Treg-mediated CD4 T cell activation which acts through active mRNA translation control to downregulate the protein dosage of protein synthesis machinery components in activated CD4 T cells. Finally, we show in a physiologically relevant setting that direct inhibition of protein synthesis using a small molecule inhibitor (RocA) can have therapeutic efficacy in alleviating unwanted inflammatory CD4 T cell activation.

\section{DISCUSSION}

Regulatory $\mathrm{T}$ cells possess the ability to potently suppress effector CD4 $\mathrm{T}$ cell responses and maintain proper immune homeostasis. Several mechanisms have been proposed for Treg-mediated suppression, all predominately focused on inhibiting aspects of effector $\mathrm{T}$ cell proliferation. Despite this emphasis, it is still unclear how target cell gene expression is modulated by Tregs. One key hallmark of CD4 $\mathrm{T}$ cell activation is the significant upregulation of global protein synthesis capacity and rate. This raises an important question as to how Tregs mediate the suppression of proliferation and activation of Teff cells. While the Treg-mediated suppression mechanisms have been heavily studied from the Tregcentered perspective yet the molecular events that happen in effector CD4 T upon Treg encounter have remained unclear(Akkaya and Shevach, 2020; Sojka et al., 2008; Vignali, 2012). To address this, we determined the effect of Tregs on post-transcriptional gene regulation in Teff cells, focusing on protein biosynthesis. We observed that Teff cells rapidly increased their global protein synthetic rate following TCR-mediated activation, and that Tregs were capable of suppressing this increase in translation at 24 hours of activation. Interestingly, we found this process is inhibited by Tregs to shut down the biosynthetic ramp-up, as early as 6 hours following $\mathrm{T}$ cell activation, required for subsequent cell division. Similarly, an acute depletion of Tregs in vivo led to the robust upregulation of protein synthesis rate in peripheral CD4 $\mathrm{T}$ cells. These data suggest that an important aspect of Treg-mediated suppression is inhibition of the ability of Teff cells to increase their biomass prior to cell division. The rapid increase in protein synthesis following acute loss of Tregs demonstrates a need for Tregs to hold in check the aberrant increase in protein synthesis of autoreactive $\mathrm{T}$ cells in the periphery. This is a highly efficient mode of regulation, inhibiting the ramp-up of biosynthesis that proceeds cell division, thereby stopping proliferation before it has begun.

We used two complementary approaches to uncover the mechanism by which Tregs suppress protein synthesis in Teff cells. We found that, upon activation, Teff cells displayed a rapid redistribution of mRNAs from monosome to polysomes, leading to increased translation. This redistribution was independent of acute changes in the transcription of the mRNAs. The vast majority of these mRNAs encoded for proteins important for protein biosynthesis, including ribosomal proteins, RNA-binding proteins, elongation, and splicing factors, suggesting that this process was involved in increasing the biomass prior to cell division. Importantly, we found that activation in the presence of Tregs inhibited the redistribution of these mRNAs, causing them to remain either free or associated with monosomes. As these changes in ribosome occupancy were independent of gene transcription and occurred within 24 
hours of stimulation, they would be undetectable by the usual methods employed to study Treg-mediated suppression-gene expression changes and cell division. Interestingly, the mRNAs affected by Tregs were enriched in 5' UTR terminal oligopyrimidine (TOP) motifs, from monosomes to polysomes in the absence of increased transcription. This motif is enriched in 5'UTRs of mRNA including ribosomal proteins and elongation factors that are core proteins of the translational machinery. This is a motif recognized by the RNA binding protein LARP1 and regulates the association of these mRNAs with stress granules(Mattijssen et al., 2021; Philippe et al., 2020). The presence of this motif on Treg-affected mRNAs suggests that Tregs may regulate the trafficking of these mRNAs from ribosomes to stress granules, thus controlling their translation.

From the perspective of the requirements for efficient Teff cell activation and clonal expansion, it is essential that activated Teff cells synthesize new proteins and increase their biomass. We observed that this process is specifically regulated by mTORC1 signaling. While it is clear that the mTOR pathway is critical for coordinating cell growth and proliferation in lymphocytes(Howie et al., 2014; So et al., 2016), we observe that Tregs specifically block mTORC1 signaling required for mRNA translation. We found that activation in the presence of Tregs led to an inhibition of S6 and 4E-BP1/2 phosphorylation, both downstream targets of mTorc1. Blockade of IL-10 signaling in the Teff cells relieved the inhibition of protein synthesis, while the addition of IL-10 during Teff stimulation (in the absence of Tregs) resulted in a reduction of global translation. Thus, IL-10 signaling is involved in Treg-mediated trans-inhibition of protein synthesis. Previous studies have shown divergent effects of IL-10 signaling on mTORC1 activity. In macrophages, IL-10 acts in a paracrine or autocrine fashion to inhibit mTORC1 activation following LPS stimulation(Baseler et al., 2016; Ip et al., 2017). The outcome was a metabolic reprogramming of the macrophages to promote oxidative phosphorylation. However, in human NK cells, IL-10 regulated metabolic changes that enhanced cellular function (e.g., IFN $\gamma$ production), and these changes were mediated via activation of mTORC1(Wang et al., 2021). While these studies show opposite effects of IL10 on cellular activity, they focus on changes in metabolism mediated through regulation of mTOR signaling. However, these studies do not address the role of IL-10 on protein synthesis.

Finally, to determine whether inhibition of translation was an effective method of regulating cellular activation we treated Treg-depleted mice with the translation inhibitor RocA. We choose RocA as it is a specific inhibitor of mRNA translation and well-tolerated in vivo. CD4 T cells in mice with acute Treg depletion display an immediate increase in global protein synthesis. Consistent with our hypothesis that inhibition of mRNA translation is an effective means of maintaining tolerance, treatment of these mice with RocA abrogated enhanced protein synthesis and inhibited induction of cytokine expression. Taken as a whole, our data show that Tregs suppress Teff cell activation, and subsequent proliferation and cytokine production, through inhibition of mTORC1, leading to suppression of protein translation through sequestration of specific mRNAs from polysomes. Our study provides a novel mechanism for the induction and maintenance of peripheral tolerance and identifies Treg derived IL-10 as one of the critical the regulators of mRNA translation affecting proliferation and activation of effector T-cells.

In this study we show that peripheral tolerance is enforced by Tregs through mRNA translational control in CD4 Teff cells. Regulatory T cells possess the ability to potently suppress effector CD4 T cell responses and maintain proper immune homeostasis. Despite the many mechanisms of suppression that have been proposed, it is still unclear how target cell gene programming is modulated by Tregs. One key 
hallmark of CD4 T cell activation is the significant upregulation of global protein synthesis capacity and rate. The major unanswered question is the mechanism through which Tregs mediate suppression that blocks proliferation and activation of Teff cells. Molecular events mediating Treg-mediated suppression of effector CD4 T have remained unclear. To address this, we interrogated if post-transcriptional gene regulation played a role in activated Teff cells undergoing Treg-mediated suppression. We observed that the Tregs were both necessary and sufficient to suppress this global rate of protein synthesis in activated Teff cells. Importantly, we found this process is inhibited by Tregs to shut down the biosynthetic ramp-up seen in the first 24 hours following $\mathrm{T}$ cell activation required for subsequent cell division. This was based on our observation that the addition of Tregs to Teff cells in vitro was sufficient to suppress overall protein synthesis rates in activated Teff cells. As mRNA translation is energetically demanding, an mTOR dependent switch to aerobic glycolysis is well known(Huang et al., 2020; Makowski et al., 2020). Recent study has shown key enzymes in the pathway are translationally regulated important for metabolic reprogramming of activated cell(Ricciardi et al., 2018). These data suggest that translation remodeling occurs earlier than metabolic reprogramming in these cells. We did not detect any mRNAs linked to metabolic processes as we probed for early events of translation remodeling. Similarly, acute depletion of Tregs in vivo led to the acute upregulation of protein synthesis rate in peripheral CD4 T cells. This suggests that there is a need for Tregs to hold in check the aberrant increase in protein synthesis of autoreactive $\mathrm{T}$ cells in the periphery. Regardless of the different mechanisms that Tregs may utilize for its suppression on Teff cells, a common theme is that changes in intracellular signaling events in Teff cells result in inhibition of proliferation. From the perspective of the requirements for efficient Teff cell activation and clonal expansion, it is essential that activated Teff cells synthesize new proteins. We observed that this process is specifically regulated by mTORC1 signaling and Tregs specifically block mTORC1 signaling required for mRNA translation. 


\section{Materials and Methods}

Mice

RiboTag (B6J.129(Cg)-Rpl22 $2^{\text {tml.1Psam }} / \mathrm{SjJ}$ : Stock\# 02997 from Jackson Labs) mice were a gift from Dr. Stanley McKnight (University of Washington(Sanz et al., 2009). These mice were either bred with Cd4Cre mice (B6.Cg-Tg(Cd4-cre)1Cwi/BfluJ: Stock\# 022071 from Jackson Labs) for mature T cellspecific RiboTag mice or CMVCre mice (B6.C-Tg(CMV-cre)1Cgn/J: Stock\# 006054) for germline whole-body RiboTag mice. For Treg-depletion studies, Foxp3-DTR mice (B6.129(Cg)-

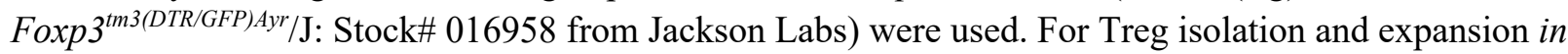

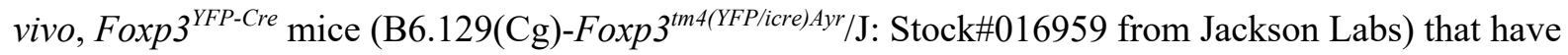
been crossed to B6 Cd45.1 mice (B6.SJL-Ptprc ${ }^{a} P e p c^{b}$ /BoyJ: Stock\# 002014 from Jackson Labs) in house were used. For the studies of IL-10 in Treg-mediated suppression of protein synthesis, 7 weeks old female of IL-10RB ${ }^{-/}$(B6.129S2-Il10rbtm1Agt/J: Stock\# 005027 from Jackson Labs) mice were used.

\section{In vivo treatments}

Foxp $3^{\text {DTR }}$ mice were intraperitoneally injected with $100 \mathrm{ng}$ of Diphtheria toxin (DT) at indicated days. For treatment of rocaglamide (RocA), mice were intraperitoneally administrated with indicated concentrations of RocA.

\section{Murine T cell isolation, culture and suppression assays}

Bulk CD4 T cells were isolated from indicated mice and stained with antibodies against CD25 and CD4 with the exception of CD4 T cells from Foxp $3^{\text {YFP-Cre }}$ mice since Foxp3+ Tregs were identified using YFP fluorescence from these mice. CD4 Teff cells devoid of Tregs (CD4+CD25-) were sorted using the BD FACS Aria sorter. Unless noted, all isolated T cells were cultured in T cell media (RPMI-1640, 10\% Fetal Bovine Serum, 2mM GlutaMAX ${ }^{\mathrm{tm}}$-I, 100U/mL Penicillin-Streptomycin, 55 $\mu \mathrm{M}$ 2-Mercaptoethanol, $1 \mathrm{mM}$ Sodium Pyruvate, $1 \mathrm{X}$ Non-Essential Amino Acids, 10mM HEPES). For Treg suppression assays, indicated numbers of tTregs (CD4+YFP+) and $1 \times 10^{5}$ CD4 Teff cells (CD4+CD25-) were cultured in round-bottom 96-well plate for the indicated times with $4 \times 10^{4}$ anti-CD3/CD28 coated magnetic beads (Teff:beads $=2.5: 1$ ). To control for T cell density, control cultures were cultured with either the same number of total Teff cells as in the Teff:Treg co-culture conditions or in some cases, CD4+YFP-CD45.1+ Teff cells sorted from Foxp $3^{Y F P \text {-Cre }}$ mice were used. To isolate ribosome-associated mRNAs from only RiboTag CD4 Teff cells (CD45.2+) after Treg co-culture, CD45.1+ Tregs were positively depleted using CD45.1-FITC and anti-FITC microbeads and running the cell mixture through LD columns (all reagents from Miltenyi Biotech). Routinely, the purity of CD45.2+ Teff cells after purification was 99.9-100\% based on flow cytometry. Ice-cold PBS and MACS buffer (0.5\% Bovine Serum Albumin fraction V, $2 \mathrm{mM}$ EDTA) were supplemented with $100 \mu \mathrm{g} / \mathrm{ml}$ cycloheximide (CHX) (Sigma, C4859) to stall elongating ribosomes throughout all isolation processes. To test proximal TCR signaling, in vitro Treg suppression assay was performed as described with modifications. CD4 Teff cells were isolated from Nur77-GFP mice and Tregs were sorted from Foxp $3^{\text {YFP-Cre }}$ mice. Prior to co-culture, CD4 Teff cells were further stained with CellTraceViolet to assess proliferative status and ensure proximal TCR signaling assessed by Nur77-GFP was prior to proliferation of these cells. Unstimulated Nur77-GFP CD4 Teff cells and Tregs from wild-type C57/B6 mice served as a negative for GFP signal. 
Ex-vivo human Treg suppression assay

We used in vitro-expanded Tregs from a single donor, and Teff (defined as CD4+CD45RA+) from 5 individual healthy donors for Treg suppression assay. Established protocols were used for Treg expansion (Long et al., 2017; Putnam et al., 2009) and Teff $\left(\mathrm{CD}^{+} \mathrm{CD}_{4}\right.$ RA $\left.{ }^{+}\right)$cells were purified PBMC using the naïve human $\mathrm{T}$ cell isolation kit from Miltenyi. To determine the effect of Tregs on overall translation in Teff we cultured Teff $\left(10^{4}\right.$ cells $)$ in absence or presence of Tregs (1:4 Treg:Teff) for 24 hours with anti-CD3/CD28 beads (at a ratio of 1:28 [bead:Teff]). Puromycin $(10 \mu \mathrm{g} / \mathrm{ml})$ was added for the last 15 minutes of culture, and then the cells were permeabilized and stained with Alexa647-coupled anti-puromycin antibody and analyzed by flow cytometry. Controls included unstimulated Teff cells also labeled with puromycin, and stimulated Teff given $\mathrm{CHX}$ at the same time as puromycin (CHX results in a complete translation blockade, so this controls for non-specific uptake of puromycin).

\section{RiboTag immunoprecipitation (Ribo-IP) and RNA isolation}

RiboTag CD4 Teff cells (or control CD4 Teff cells: RiboTag mice with no Cd4Cre expression) were washed once with ice-cold PBS with CHX and lysed in 100 $\mu$ of Polysome Lysis Buffer (PLB) (25mM Tris-HCl pH 7.5 (Ambion, AM9850G), 150mM KCl (Ambion, AM9640G), 15mM MgCl2 (Ambion, AM9530G), 1mM DTT (Sigma, 646563), 1\% NP-40 (ThermoFisher, 28324), 100 $\mu \mathrm{g} / \mathrm{ml} \mathrm{CHX,} \mathrm{100U/ml}$ SUPERaseIn RNAse Inhibitor (Ambion, AM2694), 25U/ml TurboDNAse (Ambion, AM2238), and Complete Protease Inhibitor EDTA-free (Sigma, 11836170001) in nuclease-free water (ThermoFisher, 10977015). Lysates were incubated on ice for $5 \mathrm{~min}$ and nuclei were removed by centrifugations at $200 \mathrm{~g}$, $5 \mathrm{~min}$ at $4 \mathrm{C}$ and $13,000 \mathrm{~g}, 5 \mathrm{~min}$ at $4 \mathrm{C}$. A $10 \%$ aliquot $(10 \mu \mathrm{l})$ of the lysate was harvested and kept in Trizol for total RNA extraction and the remaining lysate volume was adjusted to $400 \mu$ with PLB for even rotation with the antibodies and beads for Ribo-IP in the subsequent steps. For each sample, $3 \mu \mathrm{l}$ of anti-HA antibody (Abcam, ab9110) was added and samples were allowed to rotate at 4C for $4 \mathrm{~h}$ in a tube rotator (ThermoFisher, 88881001) at $20 \mathrm{rpm}$ speed. During rotation, $40 \mu 1$ of Dynabeads ${ }^{\mathrm{TM}}$ Protein $\mathrm{G}$ (ThermoFisher, 10004D) were prepared by washing two times in ice-cold PLB. After the anti-HA binding step, Dynabeads were added to each sample for an additional $4 \mathrm{~h}$ rotation at $4 \mathrm{C}$ (speed 20rpm). Next, samples were magnetized on the DynaMag-2 magnet (ThermoFisher, 12321D) and washed four times with $500 \mu$ l of Polysome High Salt Wash Buffer (PHSWB) $(25 \mathrm{mM}$ Tris-HCl pH 7.5, 300mM KCl, $15 \mathrm{mM}$ $\mathrm{MgCl} 2,1 \mathrm{mM}$ DTT, $1 \%$ NP-40, 100 $\mu \mathrm{g} / \mathrm{ml} \mathrm{CHX,} \mathrm{100U/ml} \mathrm{SUPERaseIn} \mathrm{RNAse} \mathrm{Inhibitor,} \mathrm{Complete}$ Protease Inhibitor EDTA-free). Usually, before the first wash, $10 \%$ of the lysate was saved to routinely check for Ribo-IP efficiency between samples by immunoblotting. For RNA extraction, Dynabeads after the final wash were resuspended in $100 \mu$ of PHSWB and homogenized in $350 \mu$ l of RLT buffer containing 40mM freshly added DTT (Qiagen RNeasy micro kit, 74004) by vigorous vortexing. Samples were left at room temperature for 10min before magnetizing the beads on the magnet and proceeding with the RNA extraction following the RNeasy micro kit protocol. At the last step, total RNA was eluted in 11 ul of nuclease-free water and RNA integrity and quantity was assessed using the Bioanalyzer 2100 total RNA pico kit. RNA integrity number (RIN) values for all samples were $>8.6$ (SFig. 2) and nextgeneration sequencing compatible cDNA libraries were generated according to the SMARTseq v4 UltraLow Input kit (Clontech, 634888). 


\section{RiboTag immunoprecipitation (Ribo-IP) and protein isolation}

Ribo-IP was performed identical to the RNA isolation method (above). After the final wash in PHSWB, Dynabeads were resuspended in 1x LDS sample buffer and heated at $65 \mathrm{C}$ for 10min. Samples were magnetized and loaded onto $12 \%$ NuPAGE Bis-Tris gel and proteins were separated by SDS-PAGE. Separated proteins were visualized using the Colloidal Blue Staining kit (ThermoFisher, LC6025) and the entire lane corresponding to each sample were excised

\section{Low input sucrose gradient polysome fractionation}

Sucrose gradients (15-60\%) were prepared in SW55Ti rotor-compatible Ultra-Clear ultracentrifuge tubes (Beckman Coulter, 344057). Briefly, a 2M (68.5\%) sucrose solution in nuclease-free water and a 10X sucrose buffer (250mM Tris-HCl pH 7.5, 1.5M KCl, 150mM MgCl2, 10mM DTT, $1 \mathrm{mg} / \mathrm{ml} \mathrm{CHX,}$ Complete protease inhibitor EDTA-free (2 Tablets per 50ml), 20U/ml SUPERaseIn RNAse Inhibitor) was prepared. Using these two solutions, sucrose solutions with $1 \mathrm{X}$ sucrose buffer and different concentrations $(60 \%, 45 \%, 30 \%, 15 \%)$ were prepared. Each solution was added from the bottom of the tubes in the following order and quantity (60\%: $750 \mu 1,45 \%: 1.5 \mathrm{ml}, 30 \%: 1.5 \mathrm{ml}, 15 \%: 750 \mu \mathrm{l})$. For each addition, tubes were kept in the $-80 \mathrm{C}$ for at least $15 \mathrm{~min}$ to freeze the sucrose solutions before adding the next sucrose solution. All tubes were sealed with parafilm and kept at $-80 \mathrm{C}$ forever. Tubes were allowed to thaw at $4 \mathrm{C}$ for 12-16h before the fractionation. Samples were prepared in PLB buffer similar to the Ribo-IP method and total RNA was quantified using RiboGreen in the low-range assay $(1 \mathrm{ng} / \mathrm{mL}-$ $50 \mathrm{ng} / \mathrm{mL}$ ). All samples were adjusted with PLB to at least 500ng of total RNA and layered carefully on top of thawed sucrose gradient tubes. Tubes were ultracentrifuged at 35,000rpm in a SW55Ti rotor using the L8-70M ultracentrifuge (acceleration: default, deceleration: 0). Separated lysates were fractionated from top to bottom with an Auto-Densi Flow fractionator (Labconco) with continuous reading of absorbance at 254nm (A254nm) using a UA-6 UV/VIS detector (Teledyne Isco). A total of 16-18 fractions $(250-300 \mu \mathrm{l})$ were fractionated with the Foxy R1 fractionator in $2 \mathrm{ml}$ Eppendorf tubes and kept on ice. For digital conversion of the A254nm signal, we attached the LabQuest Mini data-collection interface (Vernier, LQ-MINI) to the UA-6 detector. For RNA extraction, we used 3 volumes of Trizol LS to each sucrose fractions and vortexed vigorously. Each fractions were spiked-in with in vitro transcribed firefly Luciferase RNA (uncapped) to assess RNA extraction efficiency between fractions. Samples were either kept in $-80 \mathrm{C}$ at this point or proceeded with standard Trizol-mediated RNA extraction protocol or the Direct-zol 96 kit (Zymo Research, R2054) was used following the on-column DNA digestion protocol. Total RNA quantity from each fraction was measured using RiboGreen in the low-range assay to generate ribosome traces. The Bioanalyzer 2100 RNA pico kit was also used to assess the starting point of the intact $80 \mathrm{~S}$ monosome peak (containing both $28 \mathrm{~S}$ and $18 \mathrm{~S}$ rRNA bands). Equal volumes of RNA from fractions corresponding to polysomes (3 fractions after the 80S monosome peak) were pooled and digested with RQ1 DNase (Promega) and cleaned-up using RNA Clean\&Concentrator-5 kit (Zymo Research, R1013). Finally, RNA quantity (RiboGreen) and RNA quality (Bioanalyzer) were measured again before proceeding with cDNA libraries construction using the SMARTseq v4 Ultra-Low Input kit (Clontech).

\section{RNA-seq bioinformatics methods}

Reads were aligned by STAR (Dobin et al., 2012) 2.4.2a to GRCm38.91, and genes that were not expressed in at least $20 \%$ of samples were filtered out. The reads were TPM normalized and modeled using the software LIMMA ${ }^{(\text {Ritchie et al., 2015) }}$. The LIMMA model included a variable for each of the four 
mice and categorized samples by fractionation (input, polysomal, or sub-polysomal), whether they were stimulated, and their treatment (HHT, T-regulatory cells, or none). Combinations of those conditions resulted in 12 categories total. Translation efficiency was computed as the log fold-change in TPM between the polysomal and sub-polysomal fraction per condition, both at the individual mouse and aggregate levels. Differential translation efficiency was computed in LIMMA as differences between translation efficiencies between conditions. Gene set enrichment statistics were computed from LIMMAcomputed log fold-changes and the fgsea (Korotkevich et al., 2021) package.

\section{Antibody neutralization assays}

Tregs were sorted from CD45.1 Foxp3-yfp-cre mice as CD4+Foxp3(YFP)+ cells, subjected to labeled with CFSE (Thermo). Indicated number of Tregs were stimulated with anti-CD3 and anti-CD28 antibodyconjugated beads for overnight prior to suppression assay. Naïve CD4+T cells were isolated from WT mice or IL-10Rb-deficient mice using Mojo sort (biolegend), followed by staining with cell trace blue (Thermo). Indicated number of naïve $\mathrm{T}$ cells was added into the stimulated Treg cells for suppression assay. In some experiments, those naïve T cells were pre-incubated with $10 \mathrm{mg} / \mathrm{mL}$ of neutralizing antibody for IL-2R (3C7)(Biolegend), TGF- $\beta$ (1D11)( BioXcell) and/or IL-10Rb (1B1.3A)(BioXcell) for 30 min before adding Tregs and stimulation beads.

\section{Acknowledgements}

This project was funded by National Institutes of Health grants R21AI143227 (SZ \& RS), 5T32AI106677-07 (T32) and 1F32AI145283-01A1 (F32) training grant to L.S. We thank Savan and Ziegler labs for advice and input. We also thank Daniel J Campbell for his advice, Nandan Gokhale and Stephen Anderson for their critical reading and comments.

\section{Figure Legends}

Figure 1. Tregs suppress global protein synthesis in CD4 Teff cells. (A) Purified Foxp3-CD4+ Teff cells were stimulated with anti-CD3/CD28 beads with or without indicated ratio of FACS sorted Foxp3-YFP+ Tregs from Foxp ${ }^{\text {YFP-Cre }}$ mice (Treg:Teff=1:2). For cultures with CD4 Teff cells only, cell number was doubled to match total cell number in the culture $(2 \mathrm{x})$. Cells were pulsed with puromycin (PMY) for $15 \mathrm{~min}$ before harvest and intracellular staining for incorporated puromycin using a fluorophoreconjugated anti-PMY antibody. (B) PMY signal and median fluorescence intensity (MFI) was quantified. Cycloheximide (CHX) was added to $24 \mathrm{~h}$ stimulated CD4 Teff cells $5 \mathrm{~min}$ prior to PMY pulse to act as a negative control and set the baseline protein synthesis signal. (C) Purified Foxp3-CD4+ Teff cells were stimulated with anti-CD3/CD28 beads with or without indicated ratio of FACS sorted Foxp3-YFP+ Tregs from Foxp $3^{Y F P-C r e}$ mice (Treg:Teff = 1:1) for indicated times, followed by analysis of PMY incorporation as (A). (D) In vitro-expanded Tregs from a single donor, and Teff (defined as CD4 ${ }^{+} \mathrm{CD} 45 \mathrm{RA}^{+}$) from 5 individual healthy donors were used for human Treg suppression assay. Cells were co-cultured and stimulated with anti-CD3/CD28 beads for 24h and PMY staining was performed as in (A). (E) Foxp3DTR mice were injected with diphtheria toxin (DT) for two consecutive days and splenocytes were harvested on day 2. Protein synthesis in CD4+ T cells was measured by ex vivo puromycin pulsing of splenocytes and gating on CD4+ T cells. (F) CD4 Teff cells stimulated in the absence and presence of Tregs were identified by congenic markers and intracellular signaling molecules were assessed by phospho-flow cytometry. One-way ANOVA was applied for all comparisons. $* P<0.05$, $* * P<0.01$, $* * * P<0.001$. All experiments were repeated at least twice. 
Figure 2. SPEED analysis as a novel polysome profiling approach. (A) Polysome profiling approach using sucrose gradients to physically stratify cellular cytosolic lysates. Classically, continuous absorbance at $254 \mathrm{~nm}(\mathrm{~A} 254 \mathrm{~nm})$ is used to assess monosome and polysome positions. SPEED utilizes analysis of quantity and quality of total RNA from each fraction making it amenable for ultra-low biological input that is below the detection limit of A254nm reading. (B) Indicated numbers of bulk CD4 T cells were unstimulated or stimulated with anti-CD3/CD28 beads for $24 \mathrm{~h}$ and subjected for classical polysome profiling using A254nm reading to obtain polysome traces. (C) Same samples from (B) but equivalent to 500,000-1 million activated CD4 T cells and 2-3 million unstimulated CD4 T cells were subjected for polysome fractionation. No A254nm traces were obtained. Total RNA was extracted from each sucrose fractions and quantified to plot total RNA percent distribution across fractions. Total RNA quality was analyzed using Bioanalyzer (bottom Bioanalyzer results; only fractions \#1-11 were analyzed since each RNA pico chip can analyze 11 samples at a time). (D) Equal volume of RNA from each fraction was reverse transcribed into cDNA and $A c t b$ mRNA levels were quantified using qPCR. The percent $A c t b$ mRNA across fractions was quantified and plotted. Red dashed line indicates fraction \#12 in each sample.

Figure 3. Tregs specifically suppress mRNAs related to the translational machinery via active translation control. (A) Scheme to capture polysome and subpolysome-associated mRNAs for next-generation sequencing. (B) Translation efficiency (TE) was calculated as polysomal/subpolysomal enrichment for four conditions; 1) Unstimulated CD4 Teff cells, 2) 24h stimulated CD4 Teff cells, 3) 24h stimulated CD4 Teff cells co-cultured with Tregs, 4) 24h stimulated CD4 Teff cells treated with harringtonine for $30 \mathrm{~min}$. Histograms of $\log _{2}$ translation efficiency (TE) for all genes were computed for the four conditions. Genes whose $\log _{2}$ TE were different than 0 at $\leq 5 \%$ false-discovery rate (FDR) are highlighted in red. (C) Heatmap of TE in stimulated CD4 Teff cells and stimulated CD4 Teff cells co-cultured with Tregs. (D) Volcano plot of stimulated CD4 Teff cells compared to unstimulated CD4 Teff cells. 3D. Volcano plot for the differential TE of genes across stimulated and unstimulated samples. Genes. Genes whose $\log _{2} \mathrm{TE}$ were different than 0 at $\leq 5 \%$ false-discovery rate (FDR) are highlighted in red. Row-normalized $\log _{2} \mathrm{TE}$ for genes whose $\log _{2}$ TE were significantly different at $\leq 5 \%$ FDR between stimulated and unstimulated samples across all four mice. Genes highlighted in red are members of the Reactome translation pathway. (E) Barcode plot ranks genes by their log fold-change of TE between Treg-exposed stimulated cells and stimulated cells. Genes highlighted in black are genes within the Reactome Translation gene set identified to have increased their TE in the stimulated cells vs. unstimulated cell comparison. P-values are computed using the fgsea(1) package that implements Gene Set Enrichment Analysis (GSEA) statistics.

Figure 4. Tregs suppress translation of TOP motif containing mRNAs. (A) Motif discovery of Tregregulated mRNAs enriches for TOP motif. (B) mRNAs regulated by Tregs in Fig. 3 were validated using SPEED polysome qPCR. Actb mRNA served as a control. The above experiments were repeated twice.

Figure 5. IL-10 from Tregs block protein synthesis in T effector cells. (A-D) CD4 Teff cells were harvested with or without anti-CD3/CD28 beads in the presence or absence of Tregs for $24 \mathrm{~h}$, subjected for analyzing puromycin incorporation and phosphorylation of S6 and 4E-BP1. (A-C) Indicated recombinant cytokines or neutralizing antibodies for cytokines were added into the culture of CD4 Teff 
cells stimulated with anti-CD3/CD28 beads. (D) The neutralizing antibody for IL-10 receptor was added to co-culture of CD4 Teff cells and Tregs. (E) CD4 Teff cells were isolated from WT mice (black circle) and IL-10Rb-deficient mice (red circle) were harvested with or without anti-CD3/CD28 beads in the presence or absence of Tregs for $24 \mathrm{hr}$, subjected for analyzing puromycin incorporation and phosphorylation of S6 and 4E-BP1. One-way ANOVA was applied for all comparisons. ${ }^{*} P<0.05$, $* * P<0.01, * * * P<0.001$. The experiments were repeated at least twice (A-D) and once (E).

Figure 6. Acute inflammation due to Treg deficiency rescued by an mRNA translation inhibitor. (A-C) Mouse naïve CD4+ T cells were isolated from spleen of naïve C57BL/6 mice, subjected to CFSE. The cells were stimulated with anti-mouse CD3 and anti-mouse CD28-conjugated beads in the presence of indicated concentration of Roc A, FK506, or vehicle indicated as $\alpha \mathrm{CD} 3 / \mathrm{CD} 28$ in A, Stim in B, or without beads indicated as Media in A for $24 \mathrm{hr}(\mathrm{A}, \mathrm{C})$ or $72 \mathrm{hr}(\mathrm{B})$. The cells were stained for anti-CD4 (A and C) or anti-CD4 and anti-CD25 (B) for flowcytometric analysis. The data of frequency of CD25 in A was summarized in C. murine IL-2 was assayed by ELISA from supernatants collected after 24 hours after stimulation (C). (D) Incorporation of puromycin analyzed by flowcytometry post-Roc A treatment. (E-G) Foxp3-DTR mice were treated with diphtheria toxin (DT) every other day and $0.2 \mathrm{mg} / \mathrm{kg}$ of Roc A everyday started from 2 days before DT treatment, followed by isolation of splenocytes at day 8 . The cells were stained with anti-CD4, anti-TCR- $\beta$, and anti-CD44 followed by restimulation with PMA and Ionophore for $6 \mathrm{~h}$. The cells were stained by anti-IL-17A and anti-IFN- $\gamma$, followed by flowcytometric analysis (E). The bodyweight of mice at day $8(\mathrm{~F})$. The number of Th1 cells (IFN- $\gamma$ + CD44hiCD4+TCR- $\beta+$ ) and pathogenic Th17 cells (IFN- $\gamma+$ IL-17A+CD44hi CD4+TCR- $\beta+)(\mathrm{G})$. (H) Human naïve CD4+ T cells isolated from frozen PBMC, subjected to CFSE. The cells were stimulated with anti-human CD3 and anti-human CD28-conjugated beads in the presence of 10nM of Roc A or vehicle or without the beads indicated as unstim. After 3 days, cells were stained with anti-human CD25 and anti-CD4, subjected to flow cytometry for checking the frequency of divided cells and CD25+ cells. (I) Human memory Th1 cells isolated as CD4+CD25-CD45RA-CXCR3+CCR6- cells from frozen PBMC were incubated in the presence of $10 \mathrm{nM}$ of Roc A or vehicle for 2 days, followed by restimulation with PMA and Ionophore for $6 \mathrm{~h}$. The cells were stained by anti-CD4, subjected to cytoplasmic staining for IFN $\gamma$ and IL-13. The frequency of IFN- $\gamma+$ cells is summarized in the dot plots. One-way ANOVA was used for $\mathrm{C}, \mathrm{D}$ and $\mathrm{H}$; Two-tailed $t$-test (unpaired) was applied for F, G and I. $* P<0.05$, $* * P<0.01$, $* * * P<0.001$. The above experiments were repeated at least twice.

Figure 7. A model depicting Treg mediated disruption of signaling of mTORC1 that leads to the suppression of protein synthesis of mRNAs enriched for TOP motifs. 


\section{Supplementary Figures}

Supplementary Figure 1. (A) In vitro Treg suppression assay using different doses of purified Foxp3YFP+ Tregs and co-culturing with CFSE labeled YFP-CD4+ Teff cells. Data is presented in two ways. Numerating the undivided CFSE peak to show percent undivided cells. Percent suppression was calculated by measuring cells that have divided at least once. (B) CD62L expression was measured in unstimulated and stimulated CD4 Teff cells with or without Tregs at $24 \mathrm{~h}$ post stimulation. (C) Bulk CD4+ T cells were purified on day 2 from control C57/B6 mice and Foxp3-DTR mice treated with DT for two consecutive days. Cells were labeled with CFSE and activated using anti-CD3/CD28 beads. Cell size (forward scatter) and proliferation (CFSE dilution) was measured at $72 \mathrm{~h}$ post stimulation. (D) CD4 Teff cells from Nur77-GFP mice labeled with CellTraceViolet were either activated with anti-CD3/CD28 beads alone or with titrating amounts of sorted Tregs. Some conditions received increasing concentrations of RocA for eIF4A inhibition. Proximal TCR signaling was assessed using GFP in CD4 Teff cells by gating on CellTraceViolet+ population. Each datapoint represents a biological replicate (CD4 Teff cells from individual Nur77-GFP mice).

Supplementary Figure 2. (A) Non-recombined RiboTag mice (Rpl22Tag) and CMV-Cre mediated whole-body recombined RiboTag mice $\left(\mathrm{Rpl} 22^{\mathrm{HA} /+}\right.$ ) were compared for the thymic cellularity and splenic CD4, CD8 T cells and Foxp3+CD25+ Tregs. (B) Bulk CD4 T cells from T-Ribo mice were activated and subjected for fractionation on a $15-60 \%$ sucrose gradient. Proteins were precipitated from sucrose fractions and immunoblotted using anti-HA, anti-RPS6 and anti-Tubulin. Samples from RPL22 ${ }^{\Delta \mathrm{T} /+}$ show highly specific enrichment for ribosomal proteins (9-52 kDa range) as well as high molecular weight proteins that represent ribosome- associated proteins (confirmed by MS analysis). Absolute spectral counts for all RPs identified (68 out of 80). (C) Purified CD4+ T cell samples from RPL22 ${ }^{\Delta T /+}$ were subjected for Ribo-IP along with control T cells to show background immunoprecipitation.

Supplementary Figure 3. (A) CD45.1 congenically labeled Foxp3-YFP+ Tregs and Foxp3-YFP- Teff cells were co-cultured with CD45.2 CD4 Teff cells from T-Ribo mice. After 24h of stimulation, CD45.1+ cells were positively selected using magnetic activated cell sorting (MACS) using CD45.1-FITC microbeads. Pre- and post-MACS CD45.2+ percentage is plotted for all experiments. (B) Suppression of CD4 Teff cells by Tregs was measured using CFSE 72h after stimulation. (C) Specificity of Ribo-IP was tested using CD4 Teff cells from T-Ribo mice and Cre- control mice. After Ribo-IP, captured total RNA was measured using Bioanalyzer (RNA pico kit).

Supplementary Figure 4. Treg modulated translatome in CD4 Teff cells using the RiboTag approach. (A) Schematic of the RiboTag model. Exon4 of eL22 is replaced by a HA-epitope sequence containing downstream Exon4 in a Cre-dependent manner (Cd4Cre was used: T-Ribo mice). Bulk CD4 T cells were purified from $\mathrm{Cd} 4 \mathrm{Cre}$ positive and negative mice and immunoblotted for anti-HA. (B) Schematic of the translatome capture using the RiboTag for RNA-seq (C) Principal component analysis of Ribo-IP RNA samples and total input RNA samples. (D) Ribosome load (RL: Ribo-IP signal/Total input signal) was analyzed by calculating Ribo-IP mRNA signals to its corresponding total input mRNA signal in each of the condition. Density of RLs are plotted as a histogram with low RL mRNAs (number in red bracket) and high RL mRNAs (number in blue bracket). 1.5-fold cutoff was used to highlight mRNAs with 
significant RLs (red open bars) in relation to non-significant mRNAs (gray filled bars). Number of mRNAs with differential ribosome load are quantified as a histogram further supporting the idea that the RiboTag approach mainly discovers ribosome de-enriched mRNAs (negative ribosome load) as all ribosomes are captured. (D) Ribosome load was calculated for each condition and mRNAs with significant changes in ribosome (E)Venn-diagram shows that mRNAs with differential ribosome load for each condition is largely unique with minimal overlap. (F) Heatmap of Log2TMM value of mitochondrial genes and their enrichment in Ribo-IP samples. Mitochondrial genes are translated by mitoribosomes and are completely de-enriched in Ribo-IP, which further validates Ribo-IP capturing cytosolic ribosomes. (G) mRNAs showing further de-enrichment from total ribosomes (Ribo-IP) after Treg co-culture. Oneway ANOVA was applied was used for all comparisons between two groups. ${ }^{*} P<0.05, * * P<0.01$.

Supplementary Figure 5. (A) Scatterplot for each sample of quality control metrics percent alignment and median covariance of coverage, colored by fraction and treatment. Percent alignment refers to the percent of reads that can be aligned to the reference genome. Median covariance of coverage refers to the median coefficient of variation (mean/standard deviation) of gene sequence coverage for the 1000 most highly expressed transcripts. All samples pass quality control thresholds of $75 \%$ percent alignment and 0.55 median covariance of coverage. (B) Same as panel A, zoomed into the region of the graph where the samples reside. (C) Bar graph of the number of millions of reads sequenced from each sample.

Supplementary Figure 6. (A) Scatterplots showing principal components 1 and 2 for each sample across the four treatment conditions and two fractions. Principal components were computed separately for each treatment condition. (B) Scatterplots showing translation efficiency $\left(\log _{2}\right.$ fold-change of gene expression between the polysomal fraction and the subpolysomal fraction) and $-\log _{10}$ false-discovery rate for each stimulation condition. Translation efficiency false-discovery was computed using the software package LIMMA, and the false-discovery rate refers to Benjamini-Hochberg-corrected p-values for the hypotheses for each gene that the $\log _{2}$ fold-change between the fractions does not equal 0 . Genes are colored red if their $\log _{2}$ translation efficiencies are less than -1 and false-discovery rates are less than or equal to $5 \%$. Genes are colored blue if their $\log _{2}$ translation efficiencies are greater than 1 and false-discovery rates are greater than or equal to $5 \%$.

Supplementary Figure 7. Mice were treated with 0.5 or $1 \mathrm{mg} / \mathrm{kg}$ of Roc A or vehicle everyday for 7 days, followed by isolation of bone marrow and spleen at day 8. (B) Total body weight was monitored everyday post treatment. C. Incorporation of puromycin analyzed by flowcytometry post-Roc A treatment. C-F. Bone marrow cells and splenocytes were stained for neutrophils $(\mathrm{Ly} 6 \mathrm{G}+)$, eosinophils (SiglecF+CD11c-), basophils (ckit-CD200R3+FceRI+), Monocyte (CD11b+Ly6c+), naïve CD4+T (CD4+CD3+CD44lowFoxp3-), memory CD4+T (CD4+CD3+CD44hiFoxp3-), Treg $(\mathrm{CD} 4+\mathrm{CD} 3+\mathrm{Foxp} 3+), \mathrm{CD} 8 \mathrm{~T}(\mathrm{CD} 8+\mathrm{CD} 3+)$ and $\mathrm{B}(\mathrm{CD} 19+)$ cells in indicated fractions, subjected to flow cytometry.

Supplementary Table 1. A list of 149 genes, identified by SPEED, whose TE increased after stimulation at $5 \% \mathrm{FDR}$, and the $\operatorname{logFC}$ of the TE changes. These genes were examined for cis-regulatory elements shared by the 5'UTRs among Treg-sensitive mRNAs. 


\section{References}

Akkaya, B., and Shevach, E.M. (2020). Regulatory T cells: Master thieves of the immune system. Cell Immunol 355, 104160.

Araki, K., Morita, M., Bederman, A.G., Konieczny, B.T., Kissick, H.T., Sonenberg, N., and Ahmed, R. (2017). Translation is actively regulated during the differentiation of CD8(+) effector T cells. Nat Immunol 18, 1046-1057.

Bacchetta, R., Barzaghi, F., and Roncarolo, M.G. (2018). From IPEX syndrome to FOXP3 mutation: a lesson on immune dysregulation. Ann N Y Acad Sci 1417, 5-22.

Baseler, W.A., Davies, L.C., Quigley, L., Ridnour, L.A., Weiss, J.M., Hussain, S.P., Wink, D.A., and McVicar, D.W. (2016). Autocrine IL-10 functions as a rheostat for M1 macrophage glycolytic commitment by tuning nitric oxide production. Redox Biol 10, 12-23.

Bennett, C.L., Christie, J., Ramsdell, F., Brunkow, M.E., Ferguson, P.F., Whitesell, L., Kelly, T.E., Saulsbury, F.T., Chance, P.F., and Ochs, H.D. (2001). The immune dysregulation, polyendocrinopathy, enteropathy, X-linked syndrome (IPEX) is caused by mutation of FOXP3. Nature Genetics 27, 20-21.

Bjur, E., Larsson, O., Yurchenko, E., Zheng, L., Gandin, V., Topisirovic, I., Li, S., Wagner, C.R., Sonenberg, N., and Piccirillo, C.A. (2013). Distinct translational control in CD4+ T cell subsets. PLoS Genet 9, e1003494.

Bordeleau, M.E., Robert, F., Gerard, B., Lindqvist, L., Chen, S.M., Wendel, H.G., Brem, B., Greger, H., Lowe, S.W., Porco, J.A., Jr., et al. (2008). Therapeutic suppression of translation initiation modulates chemosensitivity in a mouse lymphoma model. J Clin Invest 118, 2651-2660.

Cencic, R., Carrier, M., Galicia-Vazquez, G., Bordeleau, M.E., Sukarieh, R., Bourdeau, A., Brem, B., Teodoro, J.G., Greger, H., Tremblay, M.L., et al. (2009). Antitumor activity and mechanism of action of the cyclopenta[b]benzofuran, silvestrol. PLoS One 4, e5223.

Cunningham, J.T., Moreno, M.V., Lodi, A., Ronen, S.M., and Ruggero, D. (2014). Protein and nucleotide biosynthesis are coupled by a single rate-limiting enzyme, PRPS2, to drive cancer. Cell 157, 1088-1103.

Dobin, A., Davis, C.A., Schlesinger, F., Drenkow, J., Zaleski, C., Jha, S., Batut, P., Chaisson, M., and Gingeras, T.R. (2012). STAR: ultrafast universal RNA-seq aligner. Bioinformatics 29, 15-21.

Doodes, P.D., Cao, Y., Hamel, K.M., Wang, Y., Rodeghero, R.L., Mikecz, K., Glant, T.T., Iwakura, Y., and Finnegan, A. (2010). IFN-gamma regulates the requirement for IL-17 in proteoglycan-induced arthritis. J Immunol 184, 1552-1559.

Duhen, R., Glatigny, S., Arbelaez, C.A., Blair, T.C., Oukka, M., and Bettelli, E. (2013). Cutting edge: the pathogenicity of IFN-gamma-producing Th17 cells is independent of T-bet. J Immunol 190, 4478-4482. 
Ebada, S.S., Lajkiewicz, N., Porco, J.A., Jr., Li-Weber, M., and Proksch, P. (2011). Chemistry and biology of rocaglamides (= flavaglines) and related derivatives from aglaia species (meliaceae). Prog Chem Org Nat Prod 94, 1-58.

Ernst, J.T., Thompson, P.A., Nilewski, C., Sprengeler, P.A., Sperry, S., Packard, G., Michels, T., Xiang, A., Tran, C., Wegerski, C.J., et al. (2020). Design of Development Candidate eFT226, a First in Class Inhibitor of Eukaryotic Initiation Factor 4A RNA Helicase. J Med Chem.

Fresno, M., Jimenez, A., and Vazquez, D. (1977). Inhibition of translation in eukaryotic systems by harringtonine. Eur J Biochem 72, 323-330.

Howie, D., Waldmann, H., and Cobbold, S. (2014). Nutrient Sensing via mTOR in T Cells Maintains a Tolerogenic Microenvironment. Front Immunol 5, 409.

Huang, H., Long, L., Zhou, P., Chapman, N.M., and Chi, H. (2020). mTOR signaling at the crossroads of environmental signals and T-cell fate decisions. Immunol Rev 295, 15-38.

Ip, W.K.E., Hoshi, N., Shouval, D.S., Snapper, S., and Medzhitov, R. (2017). Anti-inflammatory effect of IL-10 mediated by metabolic reprogramming of macrophages. Science 356, 513-519.

Iwasaki, S., Floor, S.N., and Ingolia, N.T. (2016). Rocaglates convert DEAD-box protein eIF4A into a sequence-selective translational repressor. Nature 534, 558-561.

Iwasaki, S., Iwasaki, W., Takahashi, M., Sakamoto, A., Watanabe, C., Shichino, Y., Floor, S.N., Fujiwara, K., Mito, M., Dodo, K., et al. (2019). The Translation Inhibitor Rocaglamide Targets a Bimolecular Cavity between eIF4A and Polypurine RNA. Mol Cell 73, 738-748 e739.

Jhanwar-Uniyal, M., Wainwright, J.V., Mohan, A.L., Tobias, M.E., Murali, R., Gandhi, C.D., and Schmidt, M.H. (2019). Diverse signaling mechanisms of mTOR complexes: mTORC1 and mTORC2 in forming a formidable relationship. Adv Biol Regul 72, 51-62.

Josefowicz, S.Z., Lu, L.F., and Rudensky, A.Y. (2012). Regulatory T cells: mechanisms of differentiation and function. Annu Rev Immunol 30, 531-564.

Kaiser, Y., Lepzien, R., Kullberg, S., Eklund, A., Smed-Sorensen, A., and Grunewald, J. (2016). Expanded lung T-bet+RORgammaT + CD4 + T-cells in sarcoidosis patients with a favourable disease phenotype. Eur Respir J 48, 484-494.

Kim, J.M., Rasmussen, J.P., and Rudensky, A.Y. (2007). Regulatory T cells prevent catastrophic autoimmunity throughout the lifespan of mice. Nat Immunol 8, 191-197.

Korotkevich, G., Sukhov, V., Budin, N., Shpak, B., Artyomov, M.N., and Sergushichev, A. (2021). Fast gene set enrichment analysis. bioRxiv, 060012 .

Lee, Y.K., Turner, H., Maynard, C.L., Oliver, J.R., Chen, D., Elson, C.O., and Weaver, C.T. (2009). Late developmental plasticity in the T helper 17 lineage. Immunity 30, 92-107. 
Leppek, K., Das, R., and Barna, M. (2018). Functional 5' UTR mRNA structures in eukaryotic translation regulation and how to find them. Nat Rev Mol Cell Biol 19, 158-174.

Li-Weber, M. (2015). Molecular mechanisms and anti-cancer aspects of the medicinal phytochemicals rocaglamides (=flavaglines). Int J Cancer 137, 1791-1799.

Long, A.E., Tatum, M., Mikacenic, C., and Buckner, J.H. (2017). A novel and rapid method to quantify Treg mediated suppression of CD4 T cells. J Immunol Methods 449, 15-22.

Makowski, L., Chaib, M., and Rathmell, J.C. (2020). Immunometabolism: From basic mechanisms to translation. Immunol Rev 295, 5-14.

Manfrini, N., Ricciardi, S., Alfieri, R., Ventura, G., Calamita, P., Favalli, A., and Biffo, S. (2020). Ribosome profiling unveils translational regulation of metabolic enzymes in primary CD4(+) Th1 cells. Dev Comp Immunol 109, 103697.

Mattijssen, S., Kozlov, G., Fonseca, B.D., Gehring, K., and Maraia, R.J. (2021). LARP1 and LARP4: up close with PABP for mRNA 3' poly(A) protection and stabilization. RNA Biol 18, 259-274.

Meyuhas, O., Thompson, E.A., Jr., and Perry, R.P. (1987). Glucocorticoids selectively inhibit translation of ribosomal protein mRNAs in P1798 lymphosarcoma cells. Mol Cell Biol 7, 2691-2699.

Ochs, H.D., Gambineri, E., and Torgerson, T.R. (2007). IPEX, FOXP3 and regulatory T-cells: a model for autoimmunity. Immunol Res 38, 112-121.

Patel, D.D. (2001). Escape from tolerance in the human X-linked autoimmunity-allergic disregulation syndrome and the Scurfy mouse. J Clin Invest 107, 155-157.

Philippe, L., van den Elzen, A.M.G., Watson, M.J., and Thoreen, C.C. (2020). Global analysis of LARP1 translation targets reveals tunable and dynamic features of 5' TOP motifs. Proc Natl Acad Sci U S A 117, 5319-5328.

Proksch, P., Giaisi, M., Treiber, M.K., Palfi, K., Merling, A., Spring, H., Krammer, P.H., and Li-Weber, M. (2005). Rocaglamide derivatives are immunosuppressive phytochemicals that target NF-AT activity in T cells. J Immunol 174, 7075-7084.

Putnam, A.L., Brusko, T.M., Lee, M.R., Liu, W., Szot, G.L., Ghosh, T., Atkinson, M.A., and Bluestone, J.A. (2009). Expansion of human regulatory T-cells from patients with type 1 diabetes. Diabetes 58, 652662.

Ricciardi, S., Manfrini, N., Alfieri, R., Calamita, P., Crosti, M.C., Gallo, S., Muller, R., Pagani, M., Abrignani, S., and Biffo, S. (2018). The Translational Machinery of Human CD4(+) T Cells Is Poised for Activation and Controls the Switch from Quiescence to Metabolic Remodeling. Cell Metab 28, 895-906 e895. 
Ritchie, M.E., Phipson, B., Wu, D., Hu, Y., Law, C.W., Shi, W., and Smyth, G.K. (2015). limma powers differential expression analyses for RNA-sequencing and microarray studies. Nucleic Acids Res 43, e47.

Rogers, G.W., Jr., Komar, A.A., and Merrick, W.C. (2002). eIF4A: the godfather of the DEAD box helicases. Prog Nucleic Acid Res Mol Biol 72, 307-331.

Sanz, E., Yang, L., Su, T., Morris, D.R., McKnight, G.S., and Amieux, P.S. (2009). Cell-type-specific isolation of ribosome-associated mRNA from complex tissues. Proc Natl Acad Sci U S A 106, 1393913944.

Schmidt, E.K., Clavarino, G., Ceppi, M., and Pierre, P. (2009). SUnSET, a nonradioactive method to monitor protein synthesis. Nat Methods 6, 275-277.

Schneider, A., Long, S.A., Cerosaletti, K., Ni, C.T., Samuels, P., Kita, M., and Buckner, J.H. (2013). In active relapsing-remitting multiple sclerosis, effector T cell resistance to adaptive T(regs) involves IL-6mediated signaling. Sci Transl Med 5, 170ra115.

Seedhom, M.O., Hickman, H.D., Wei, J., David, A., and Yewdell, J.W. (2016). Protein Translation Activity: A New Measure of Host Immune Cell Activation. J Immunol 197, 1498-1506.

Shevach, E.M. (2009). Mechanisms of foxp3+ T regulatory cell-mediated suppression. Immunity 30, 636645.

So, L., Lee, J., Palafox, M., Mallya, S., Woxland, C.G., Arguello, M., Truitt, M.L., Sonenberg, N., Ruggero, D., and Fruman, D.A. (2016). The 4E-BP-eIF4E axis promotes rapamycin-sensitive growth and proliferation in lymphocytes. Sci Signal 9, ra57.

Sojka, D.K., Huang, Y.H., and Fowell, D.J. (2008). Mechanisms of regulatory T-cell suppression - a diverse arsenal for a moving target. Immunology 124, 13-22.

Tang, Q., and Bluestone, J.A. (2008). The Foxp3+ regulatory T cell: a jack of all trades, master of regulation. Nat Immunol 9, 239-244.

Thoreen, C.C., Chantranupong, L., Keys, H.R., Wang, T., Gray, N.S., and Sabatini, D.M. (2012). A unifying model for mTORC1-mediated regulation of mRNA translation. Nature 485, 109-113.

Truitt, M.L., Conn, C.S., Shi, Z., Pang, X., Tokuyasu, T., Coady, A.M., Seo, Y., Barna, M., and Ruggero, D. (2015). Differential Requirements for eIF4E Dose in Normal Development and Cancer. Cell 162, 5971.

Vignali, D.A. (2012). Mechanisms of T(reg) Suppression: Still a Long Way to Go. Front Immunol 3, 191.

Vignali, D.A., Collison, L.W., and Workman, C.J. (2008). How regulatory T cells work. Nat Rev Immunol 8, 523-532. 
Wang, R., Dillon, C.P., Shi, L.Z., Milasta, S., Carter, R., Finkelstein, D., McCormick, L.L., Fitzgerald, P., Chi, H., Munger, J., et al. (2011). The transcription factor Myc controls metabolic reprogramming upon T lymphocyte activation. Immunity 35, 871-882.

Wang, Z., Guan, D., Huo, J., Biswas, S.K., Huang, Y., Yang, Y., Xu, S., and Lam, K.P. (2021). IL-10 Enhances Human Natural Killer Cell Effector Functions via Metabolic Reprogramming Regulated by mTORC1 Signaling. Front Immunol 12, 619195.

Wardman, J.H., Gomes, I., Bobeck, E.N., Stockert, J.A., Kapoor, A., Bisignano, P., Gupta, A., Mezei, M., Kumar, S., Filizola, M., et al. (2016). Identification of a small-molecule ligand that activates the neuropeptide receptor GPR171 and increases food intake. Sci Signal 9, ra55.

Wildin, R.S., Ramsdell, F., Peake, J., Faravelli, F., Casanova, J.L., Buist, N., Levy-Lahad, E., Mazzella, M., Goulet, O., Perroni, L., et al. (2001). X-linked neonatal diabetes mellitus, enteropathy and endocrinopathy syndrome is the human equivalent of mouse scurfy. Nature Genetics 27, 18-20.

Wolf, T., Jin, W., Zoppi, G., Vogel, I.A., Akhmedov, M., Bleck, C.K.E., Beltraminelli, T., Rieckmann, J.C., Ramirez, N.J., Benevento, M., et al. (2020). Dynamics in protein translation sustaining T cell preparedness. Nat Immunol 21, 927-937.

Zoncu, R., Efeyan, A., and Sabatini, D.M. (2011). mTOR: from growth signal integration to cancer, diabetes and ageing. Nat Rev Mol Cell Biol 12, 21-35. 
Figure 1: Tregs suppress global protein synthesis in CD4 Teff cells.
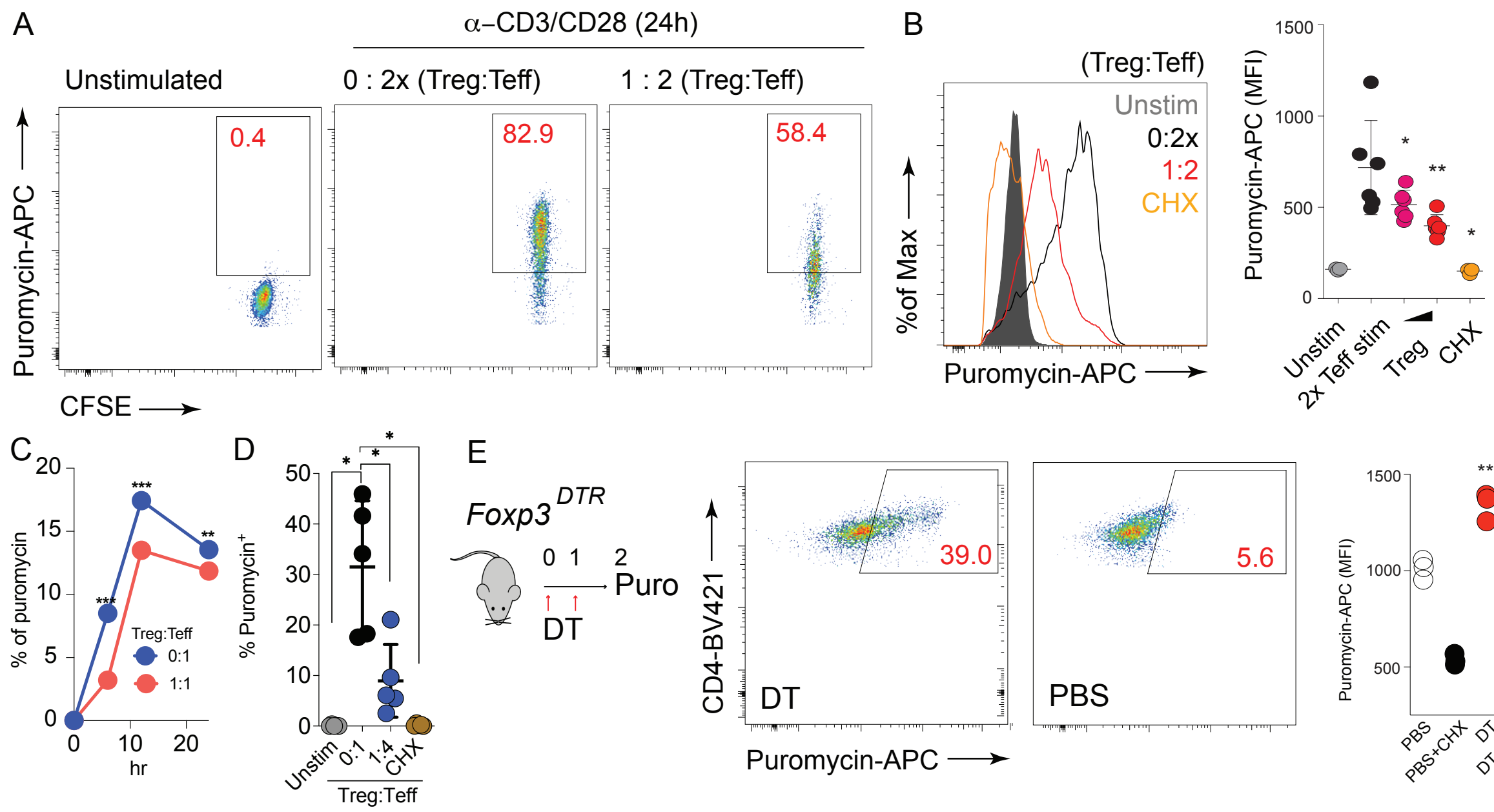

$\mathrm{E}$

$\mathrm{F}$
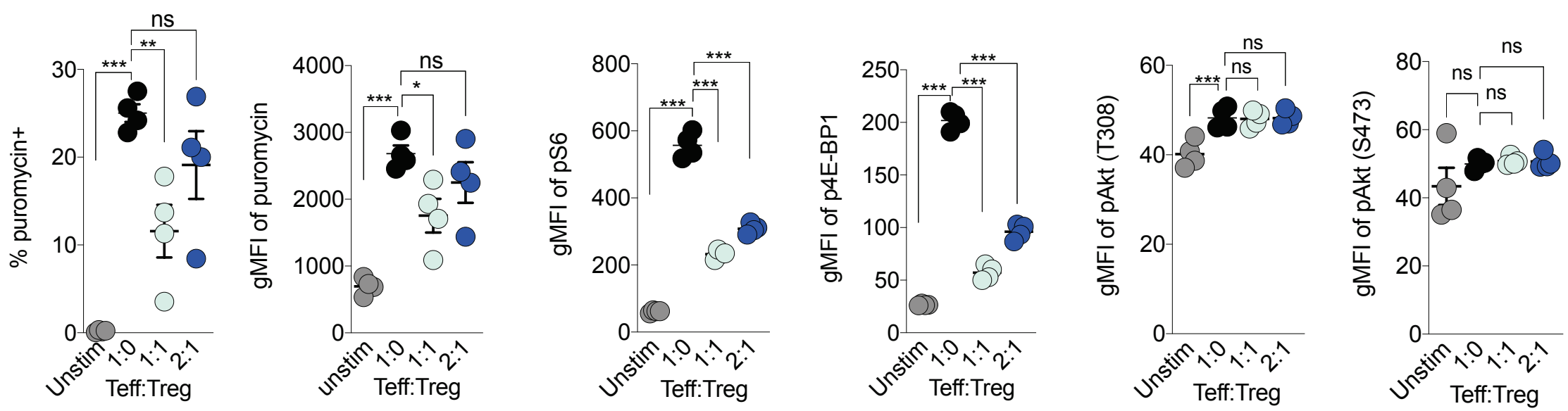
Figure 2: SPEED analysis as a novel polysome profiling approach.

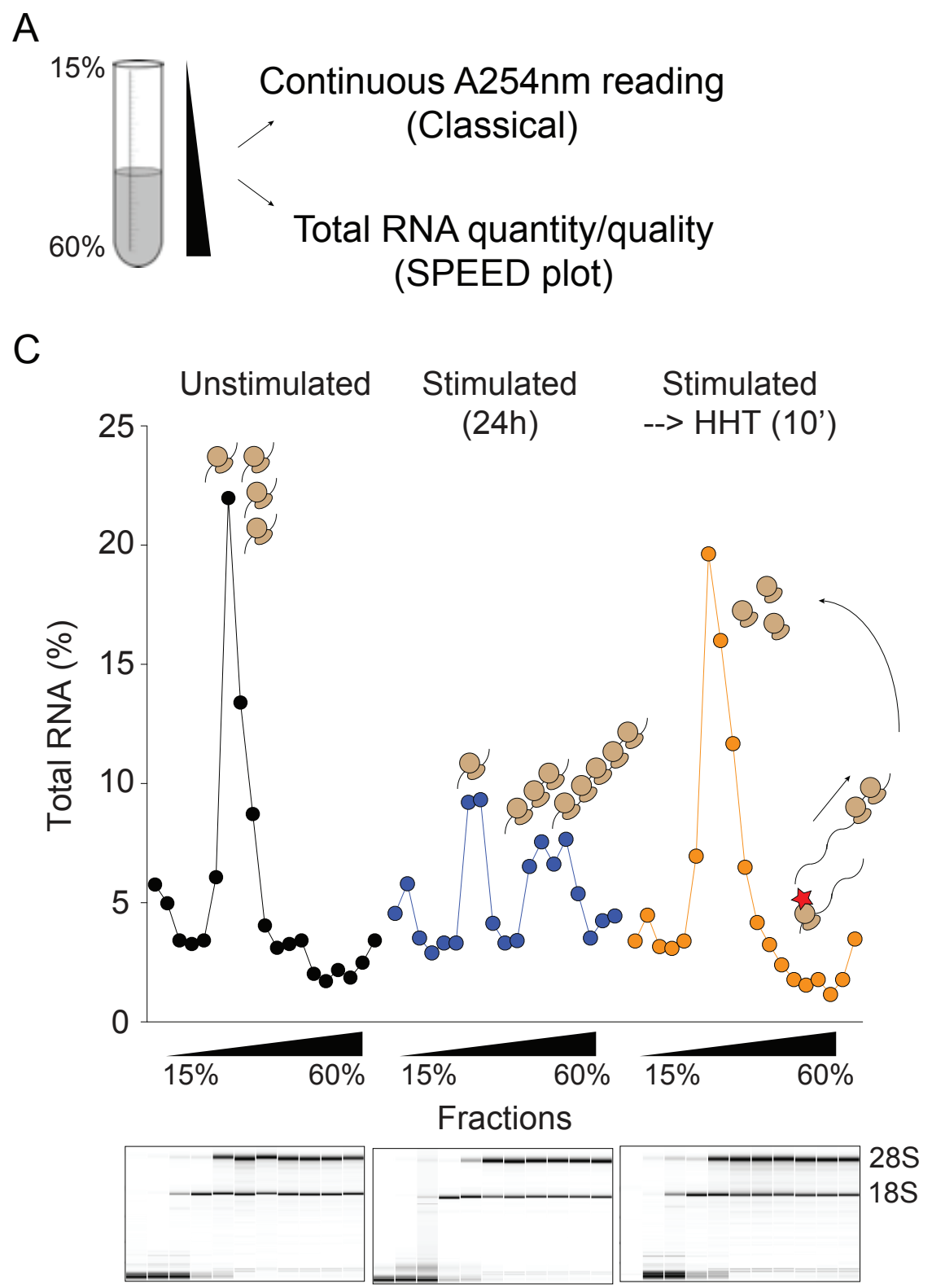

B

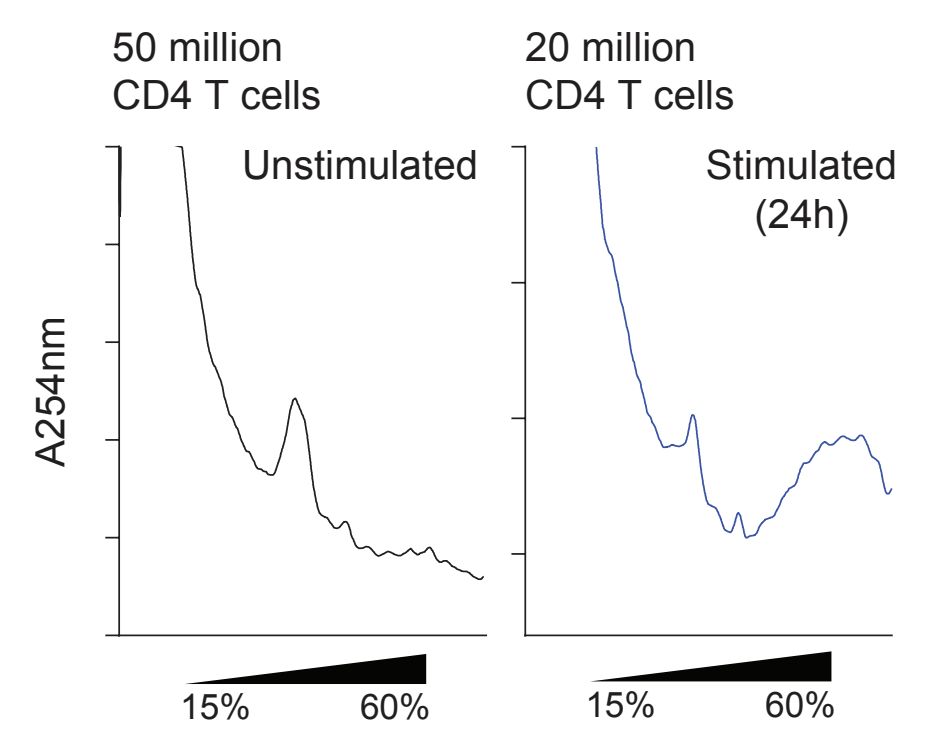

D Unstimulated Stimulated (24h) Stimulated -> HHT (10')

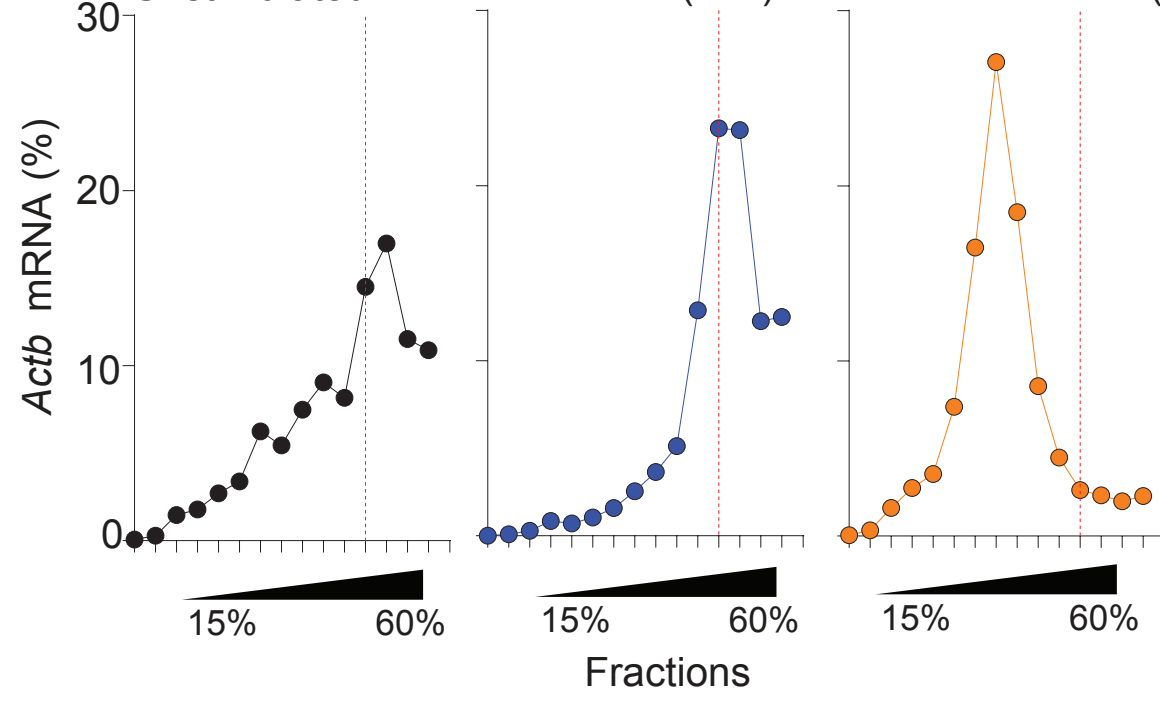


Figure 3: Tregs specifically suppress mRNAs related to the translational machinery via active translation control.

A

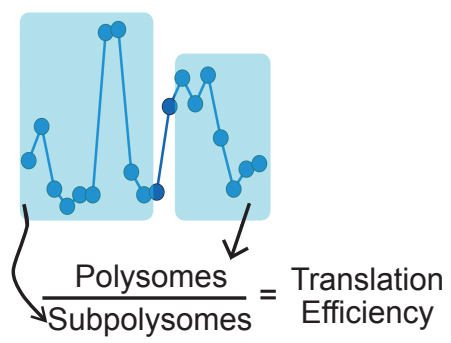

C

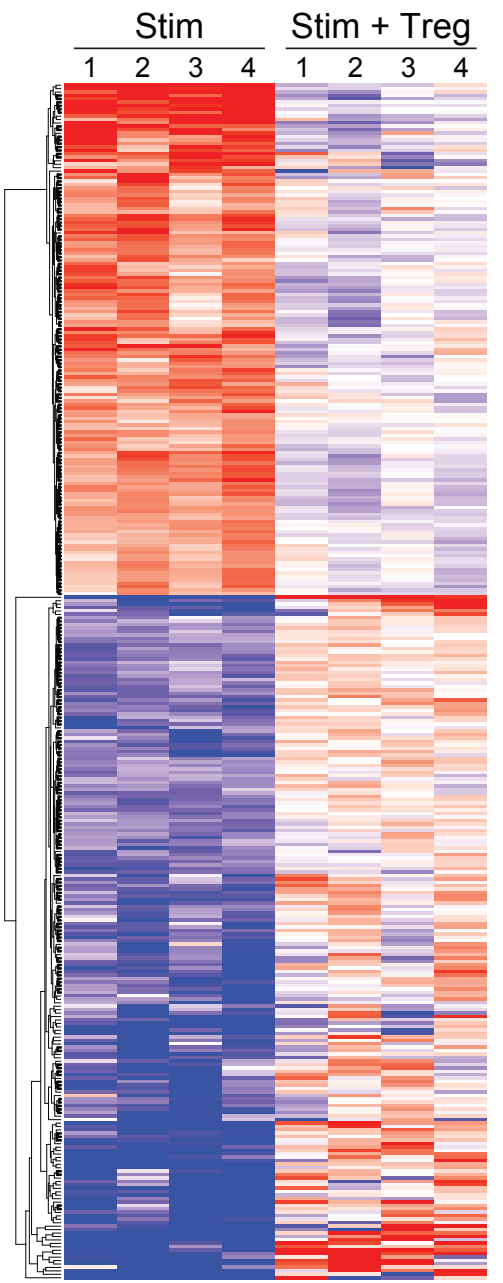

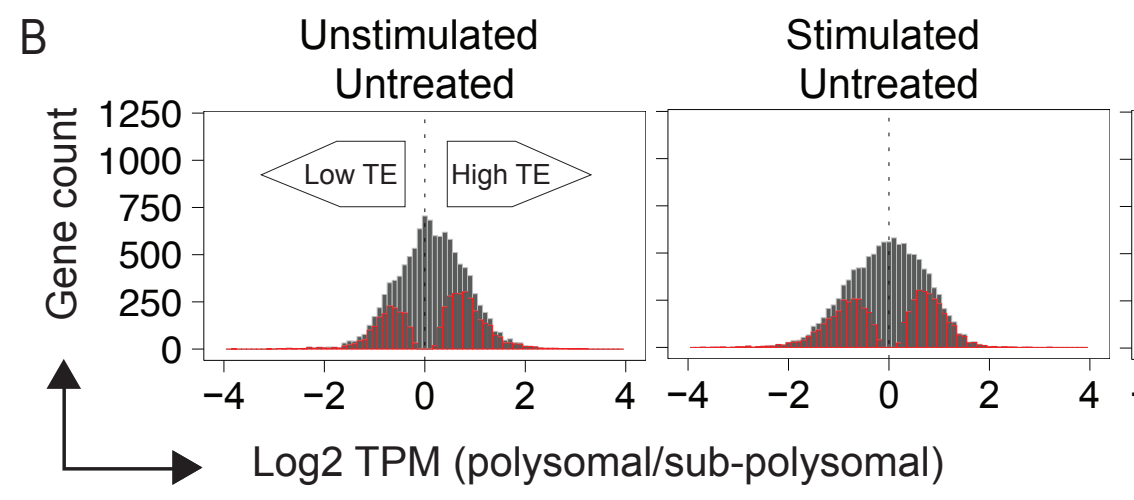

Stimulated

Treg-treated

Stimulated HHT-treated

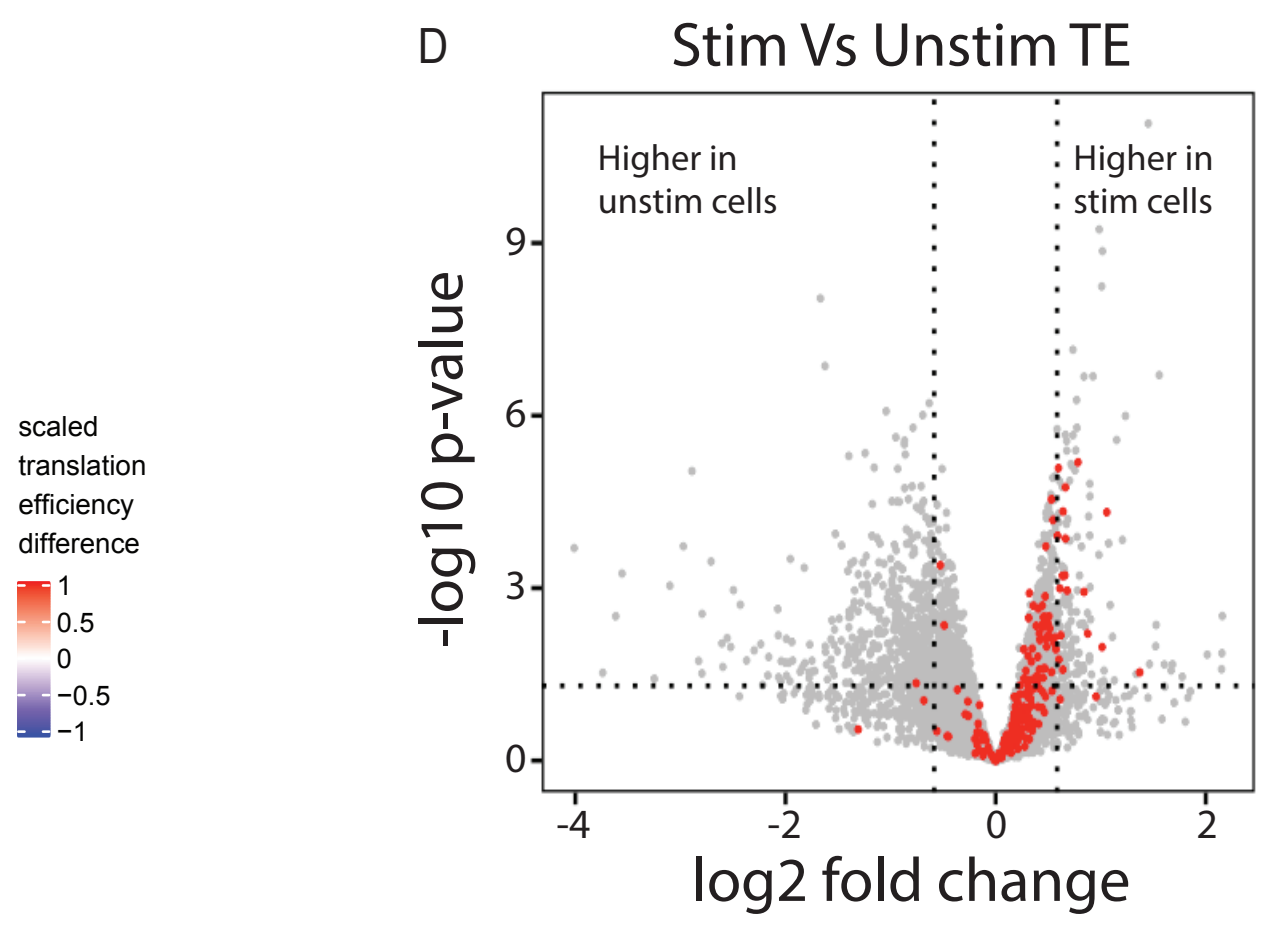

E

Log2 Translation Efficiency Difference Treg exposed stimulated vs. stimulated cells Reactome Translation Genes
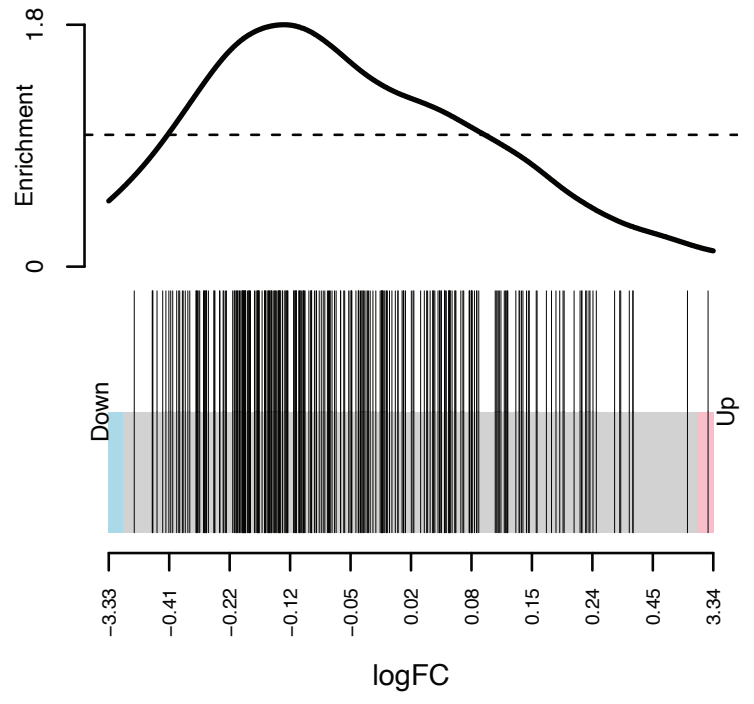

\begin{tabular}{|l|c|c|c|c|c|c|}
\hline \multicolumn{1}{|c|}{ pathway } & pval & padj & ES & NES & nMoreExtreme & size \\
\hline REACTOME_TRANSLATION & 0.01081799 & 0.01081799 & -0.3201563 & -1.324407 & 398 & 286 \\
\hline Stim TE up & $2.47 \mathrm{E}-05$ & $3.71 \mathrm{E}-05$ & -0.5691487 & -2.1805147 & 0 & 149 \\
\hline Stim TE down & $1.64 \mathrm{E}-05$ & $3.71 \mathrm{E}-05$ & 0.54754491 & 2.08292609 & 0 & 199 \\
\hline
\end{tabular}


Figure 4. Tregs suppress translation of TOP motif containing mRNAs.

A

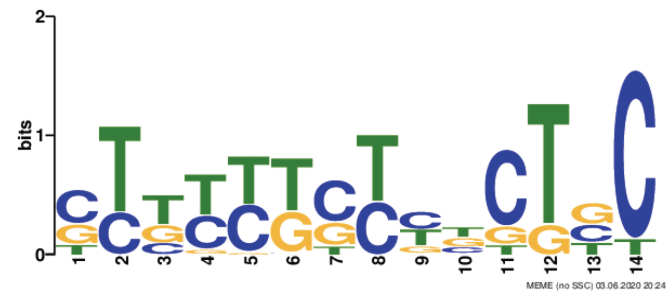

B
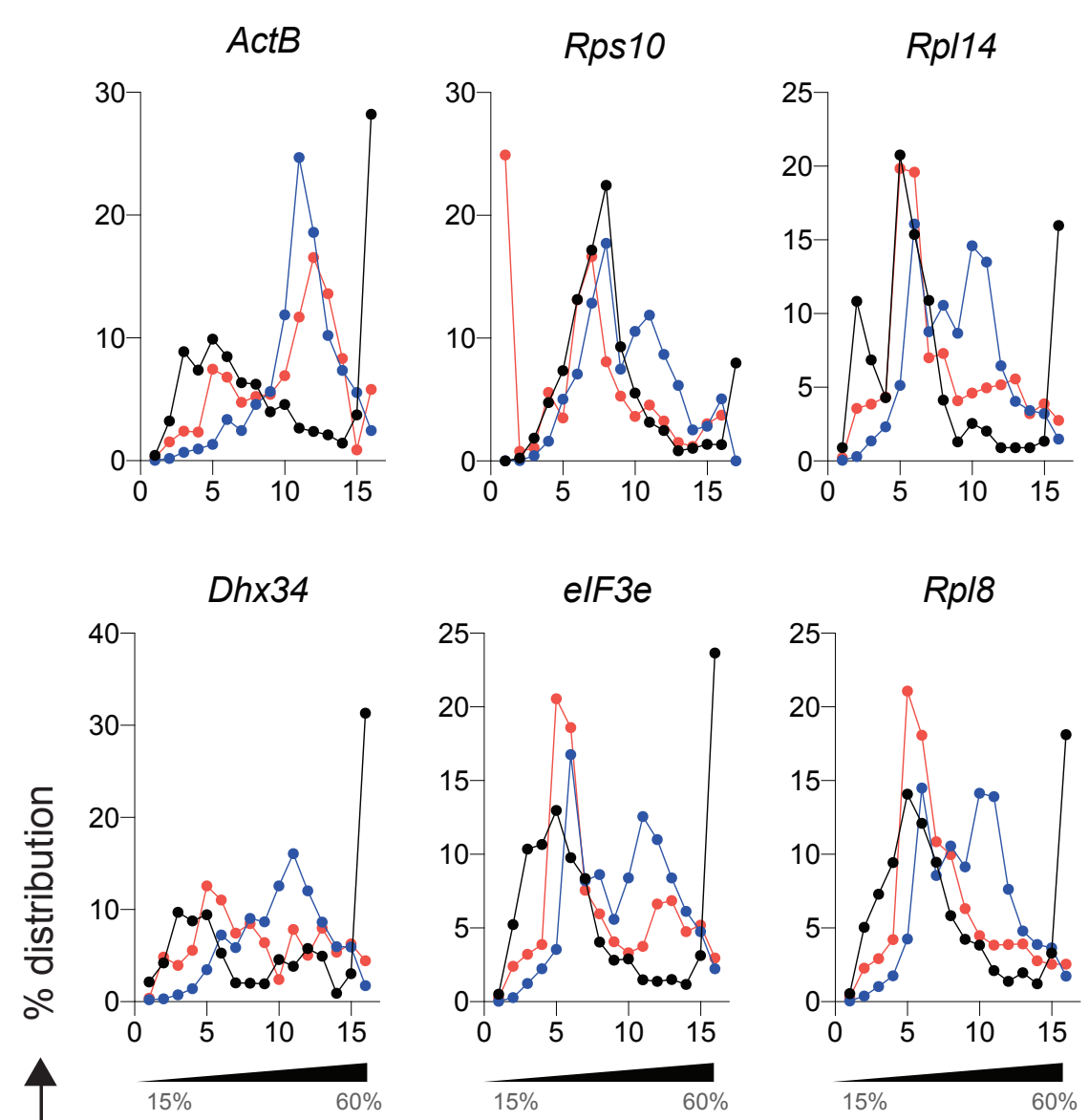

Fraction number

$\rightarrow$ Unstim

$\rightarrow$ Stim

$\multimap$ Stim + iTreg 
Figure 5. Tregs suppress translation of TOP motif containing mRNAs.
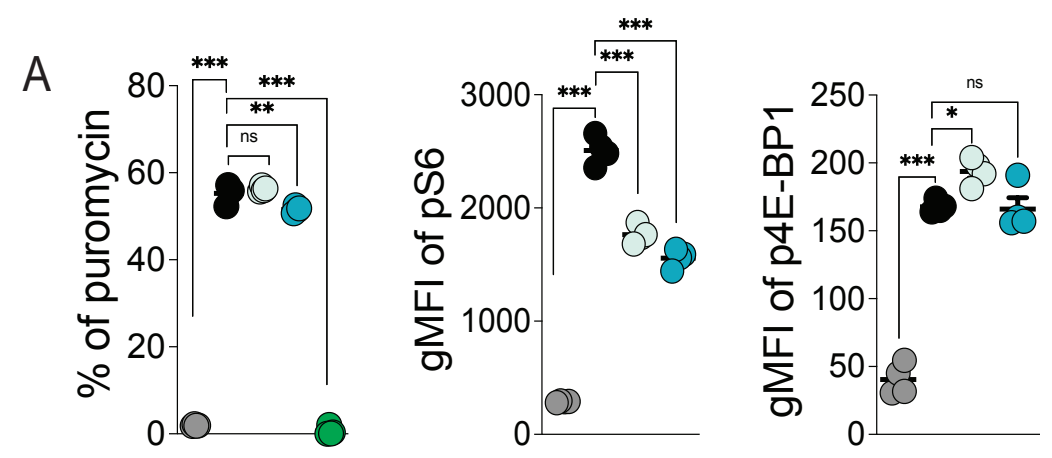

unstim

stim

JSE6-1A12 (alL-2) $10 \mu \mathrm{g} / \mathrm{mL}$

$3 \mathrm{C} 7(\alpha \mathrm{CD} 25) 10 \mu \mathrm{g} / \mathrm{mL}$

chx

B
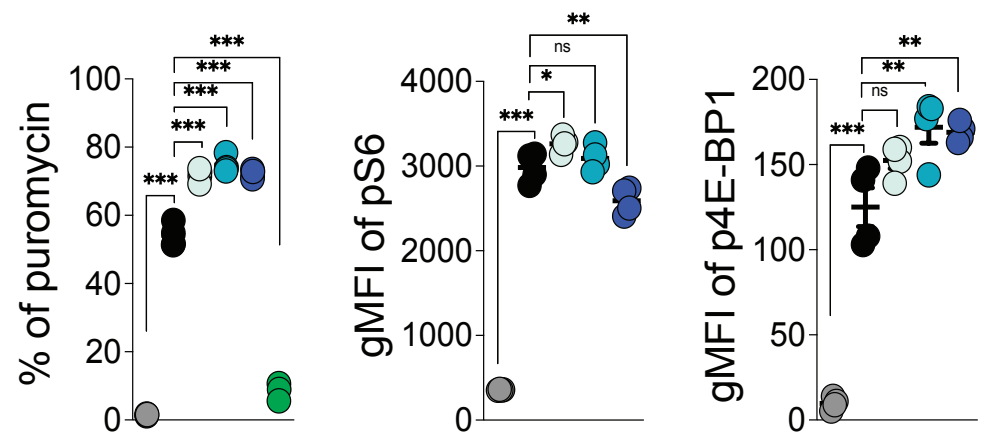

unstim

stim

$1 \mathrm{ng} / \mathrm{mL}$ rTGF $\beta$

$3 \mathrm{ng} / \mathrm{mL}$ rTGF $\beta$

$10 \mathrm{ng} / \mathrm{mL}$ rTGF $\beta$

chx

C
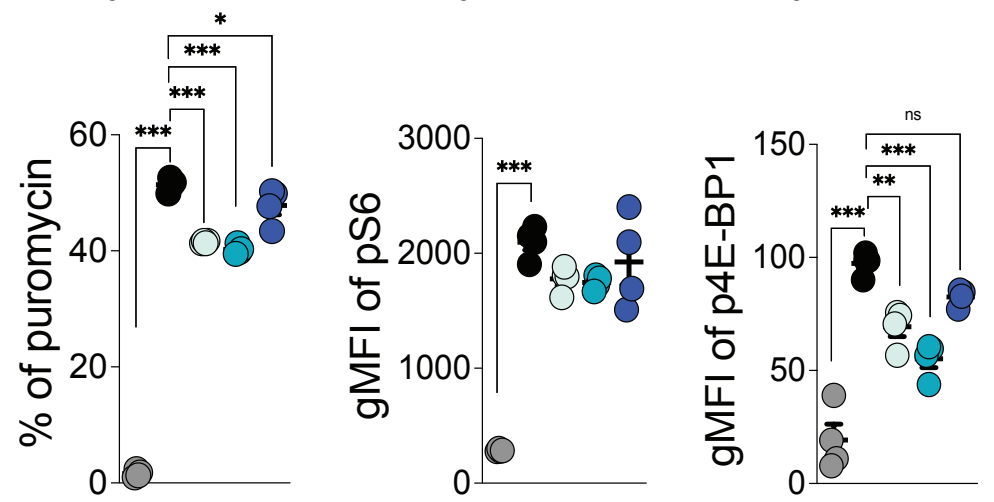

unstim

stim

$1 \mathrm{ng} / \mathrm{mL}$ rlL-10

$3 \mathrm{ng} / \mathrm{mL}$ rlL-10

$10 \mathrm{ng} / \mathrm{mL}$ rlL-10
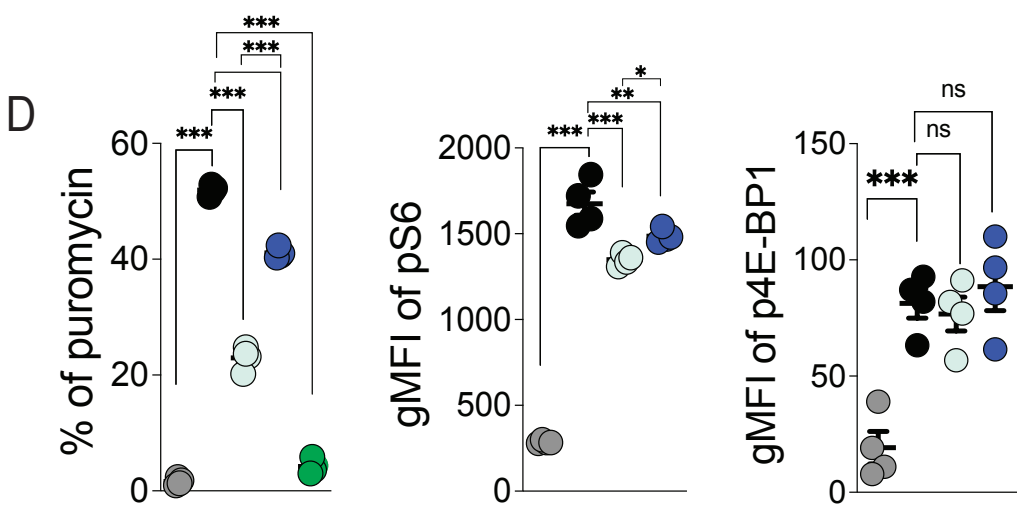

unstim

stim

1:1 Teff:Treg

1:1 Teff:Treg $+\alpha \mathrm{LL}-10 \mathrm{R}$

ch $x$

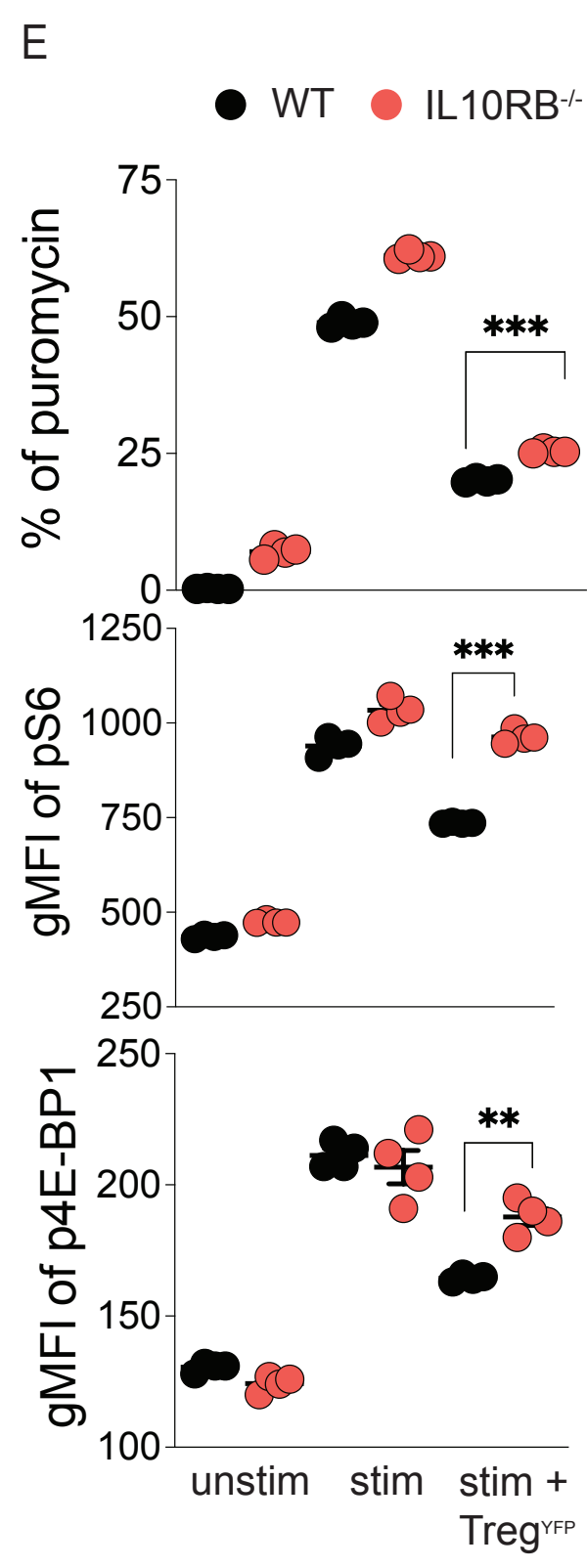


Figure 6. Acute inflammation due to Treg deficiency rescued by an mRNA translation inhibitor.

A 24h stim (CD4+)

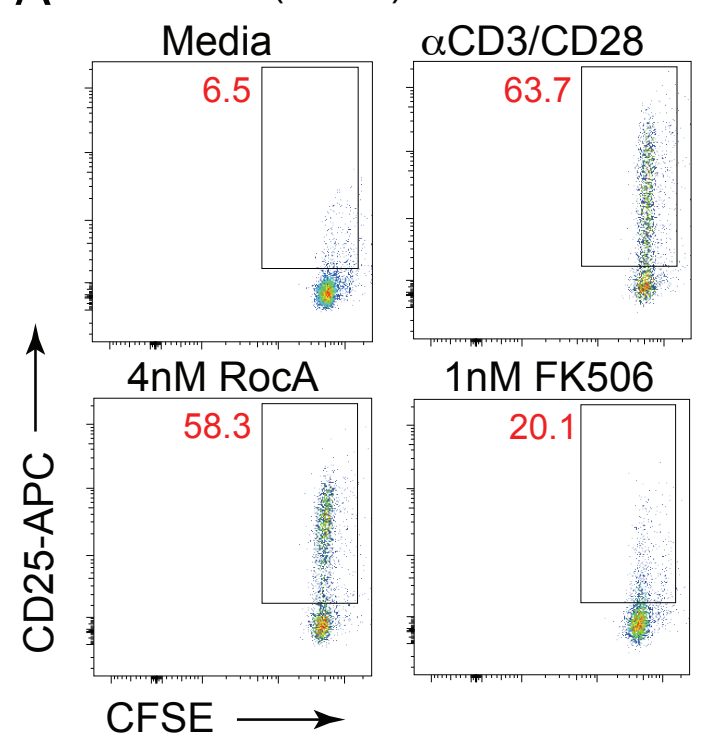

B 72h stim (CD4+)

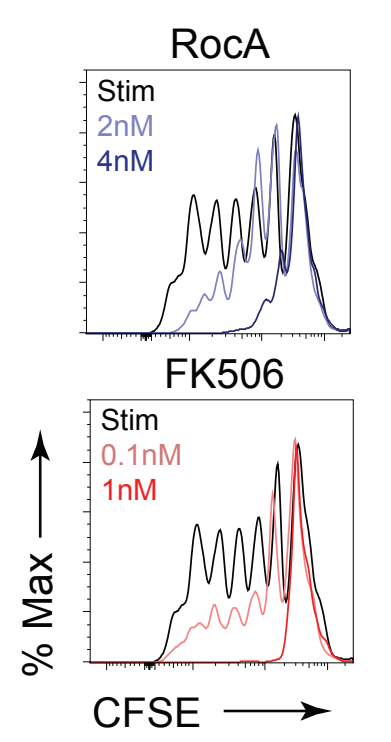

E

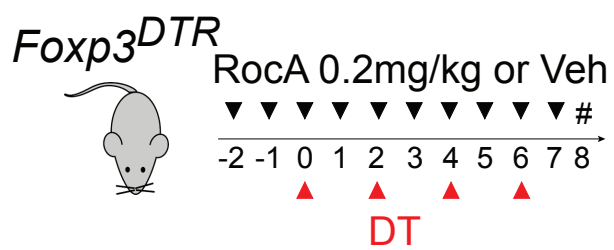

PMA+lono (D8)

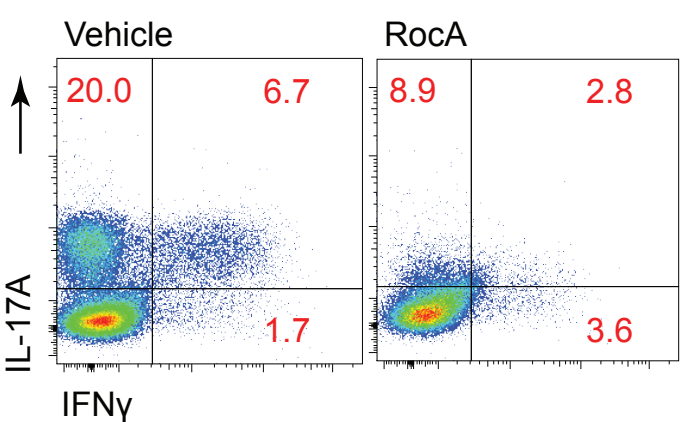

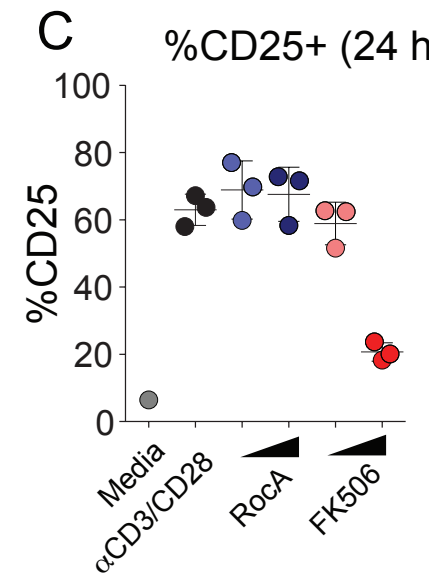

$\mathrm{H}$

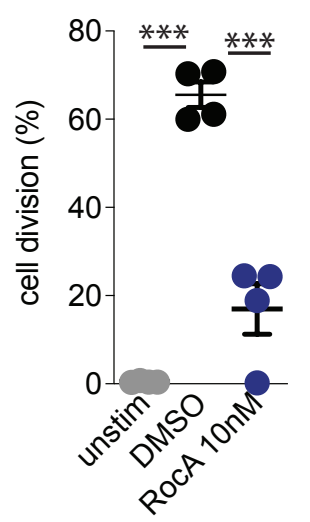

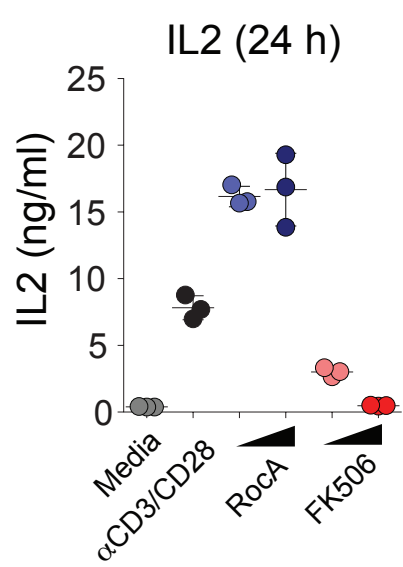

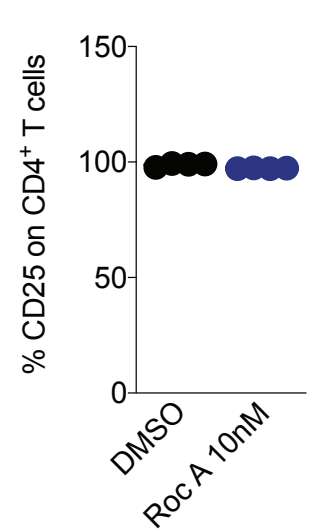

D
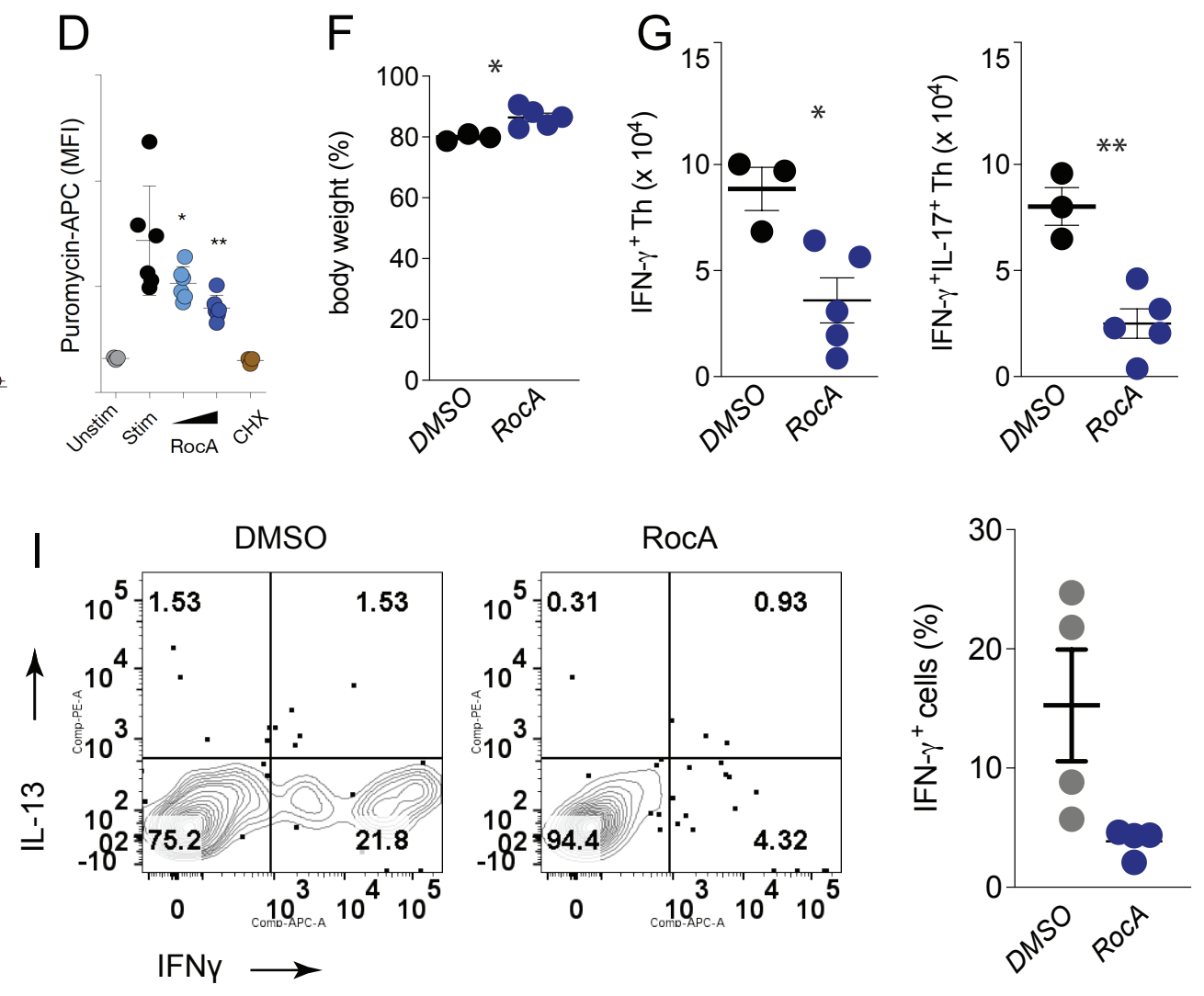
Figure 7. A model depicting Treg mediated disruption of signaling of mTORC1 that leads to the suppression of protein synthesis of mRNAs enriched for TOP motifs.

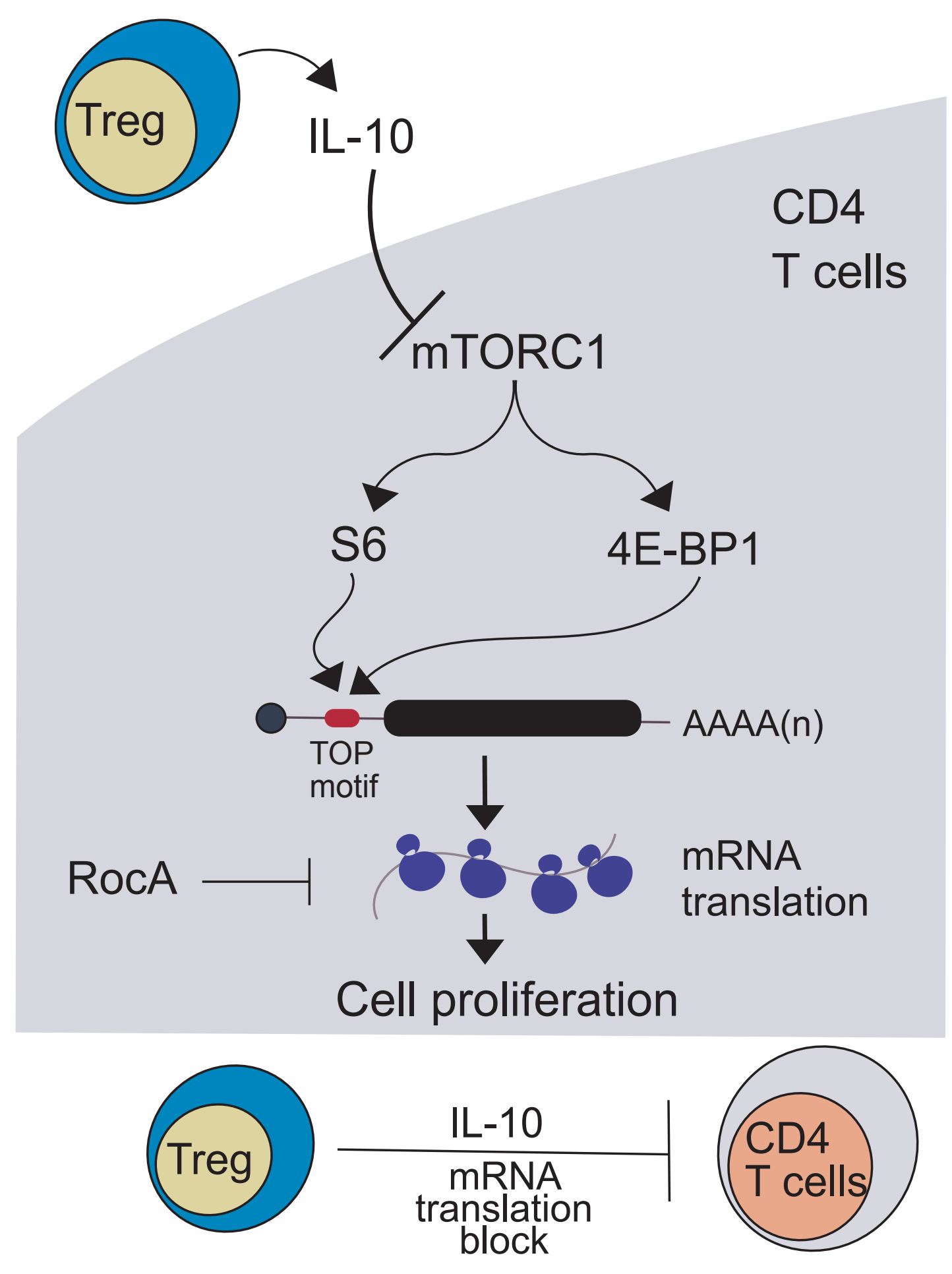




\section{S Fig 1}

A Treg suppression ( $n=3)$
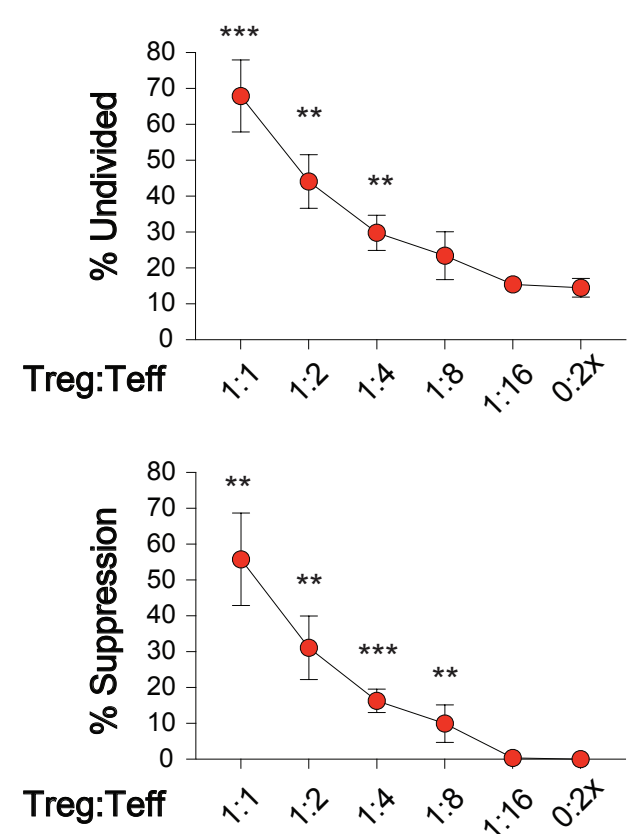

C

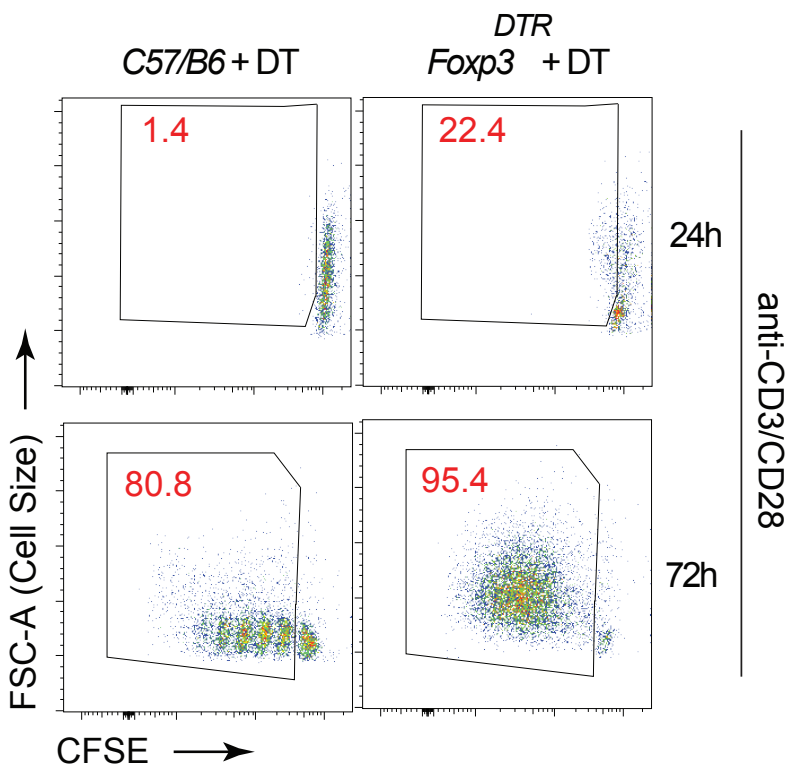

B
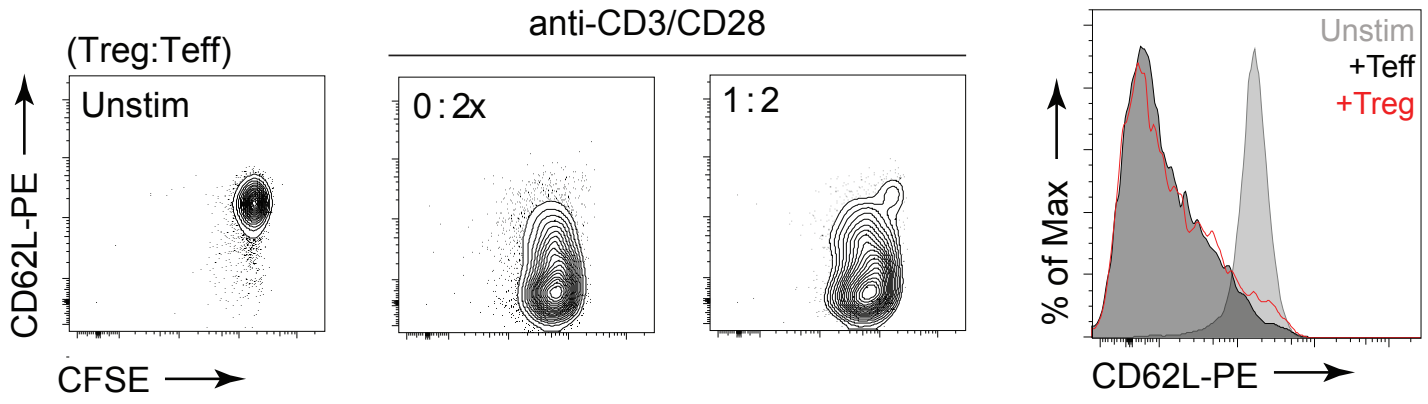

D

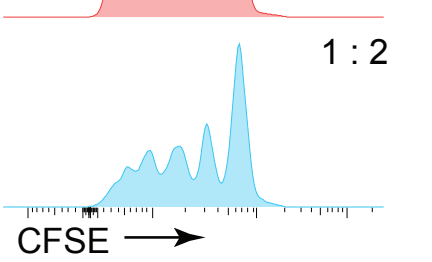

D Unstim

Teff:Treg (1:0)

Teff:Treg (1:1)

Teff:Treg (2:1)
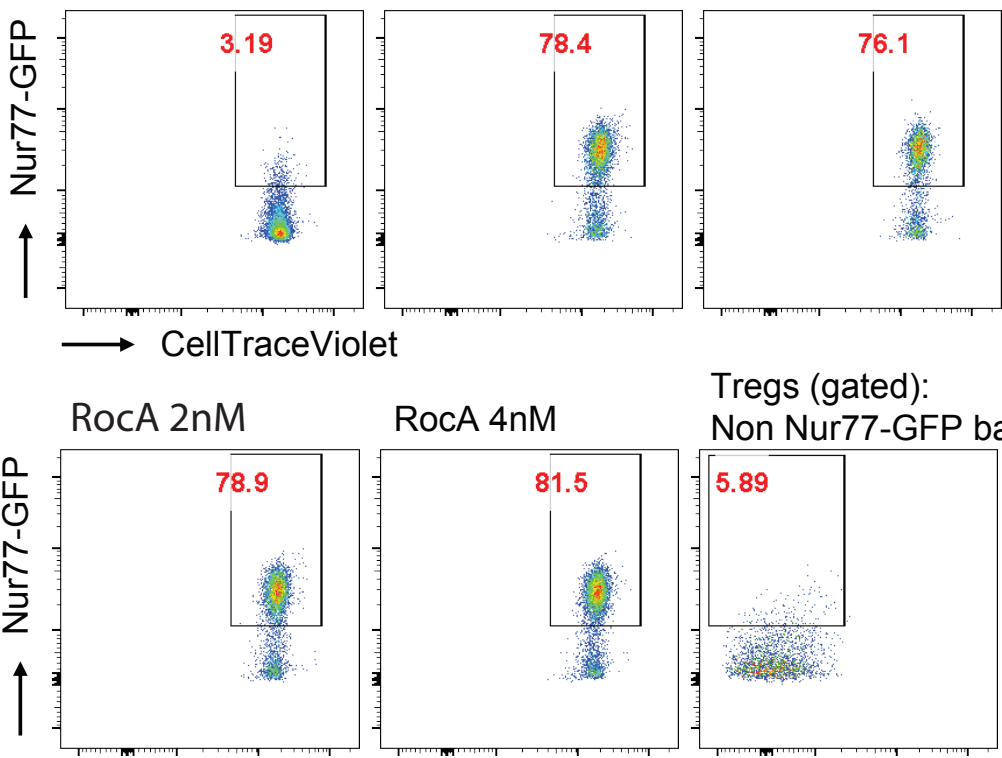

$\longrightarrow$ CellTraceViolet

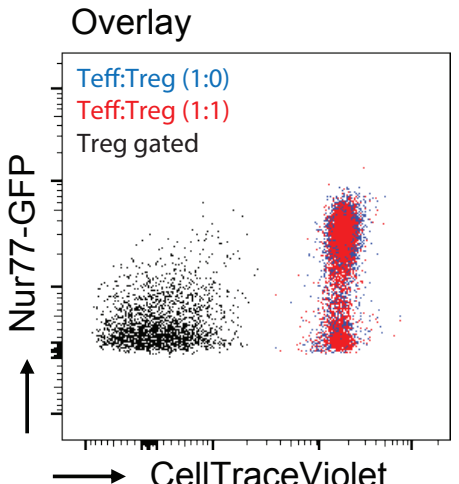

- Unstim

- Stimulated $24 \mathrm{~h}$

- Treg 1:2

- Treg 1:1

- RocA 2nM

- RocA 4nM 
A

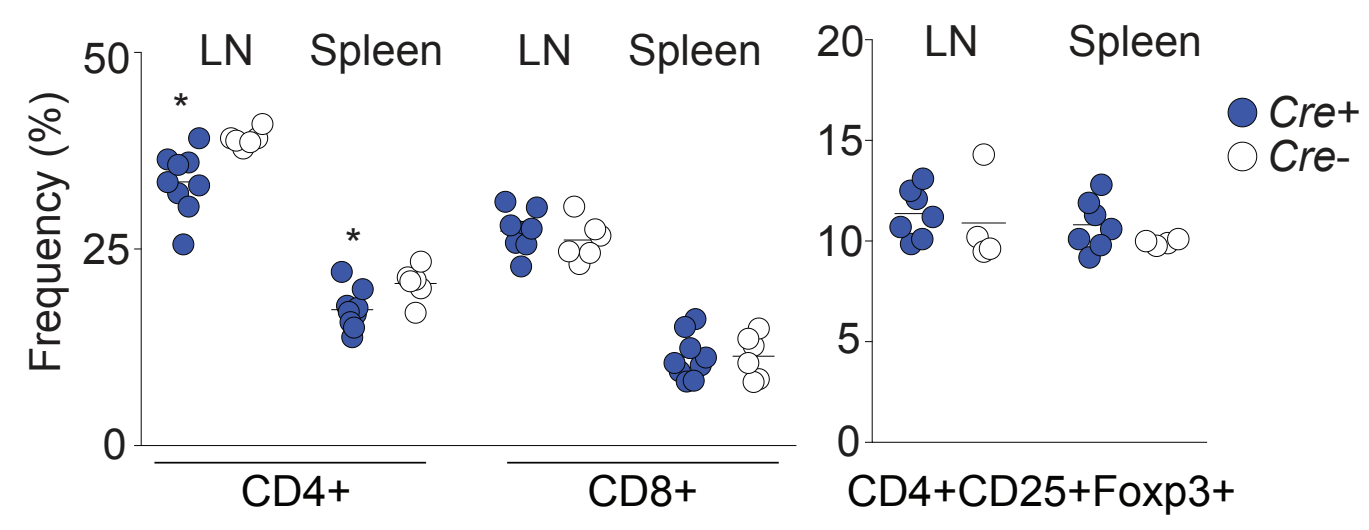

B Activated CD4 T cells (36h)

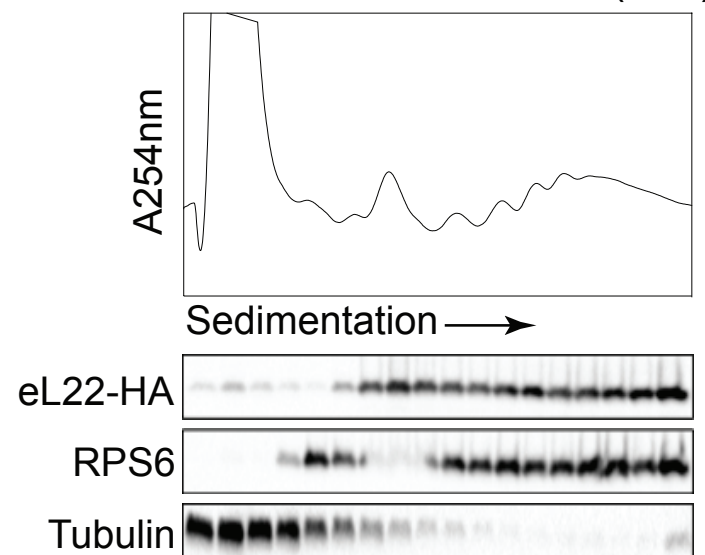

D

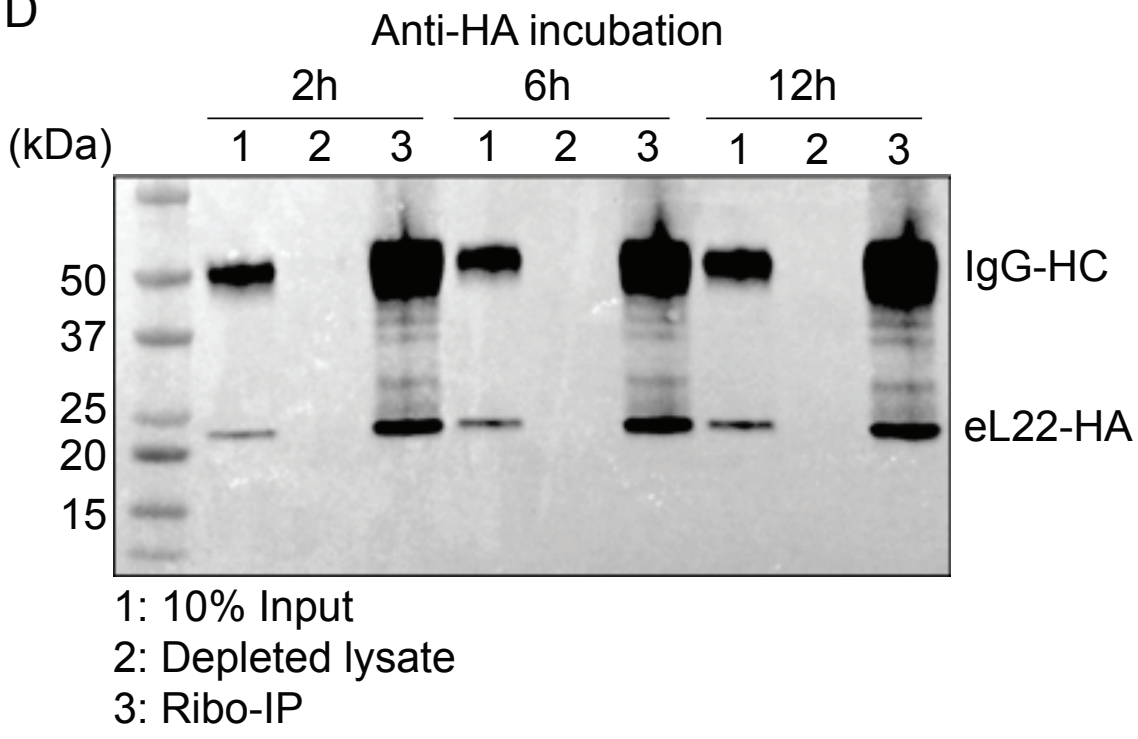

C Input $\frac{(10 \%)}{+-} \frac{\text { Ribo-IP }}{+- \text { Cre }}$

50

37

25

20

15

(kDa)

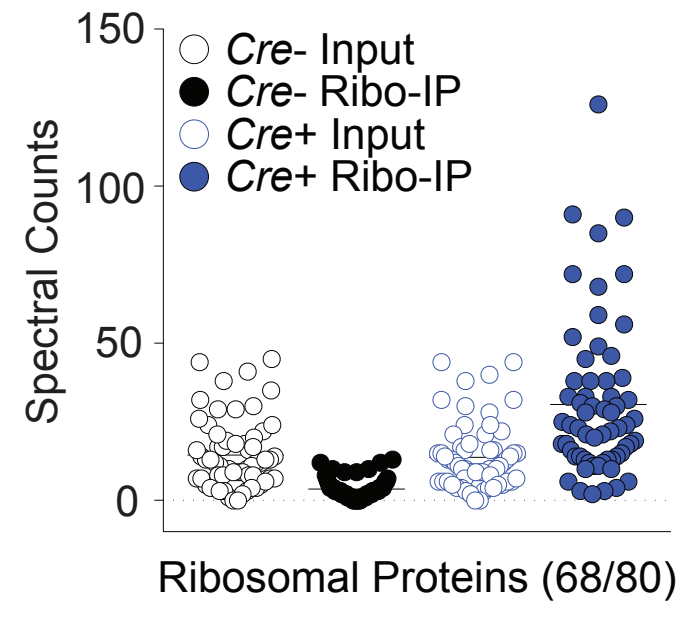

Ribosomal Proteins (68/80) 


\section{S Fig 3}
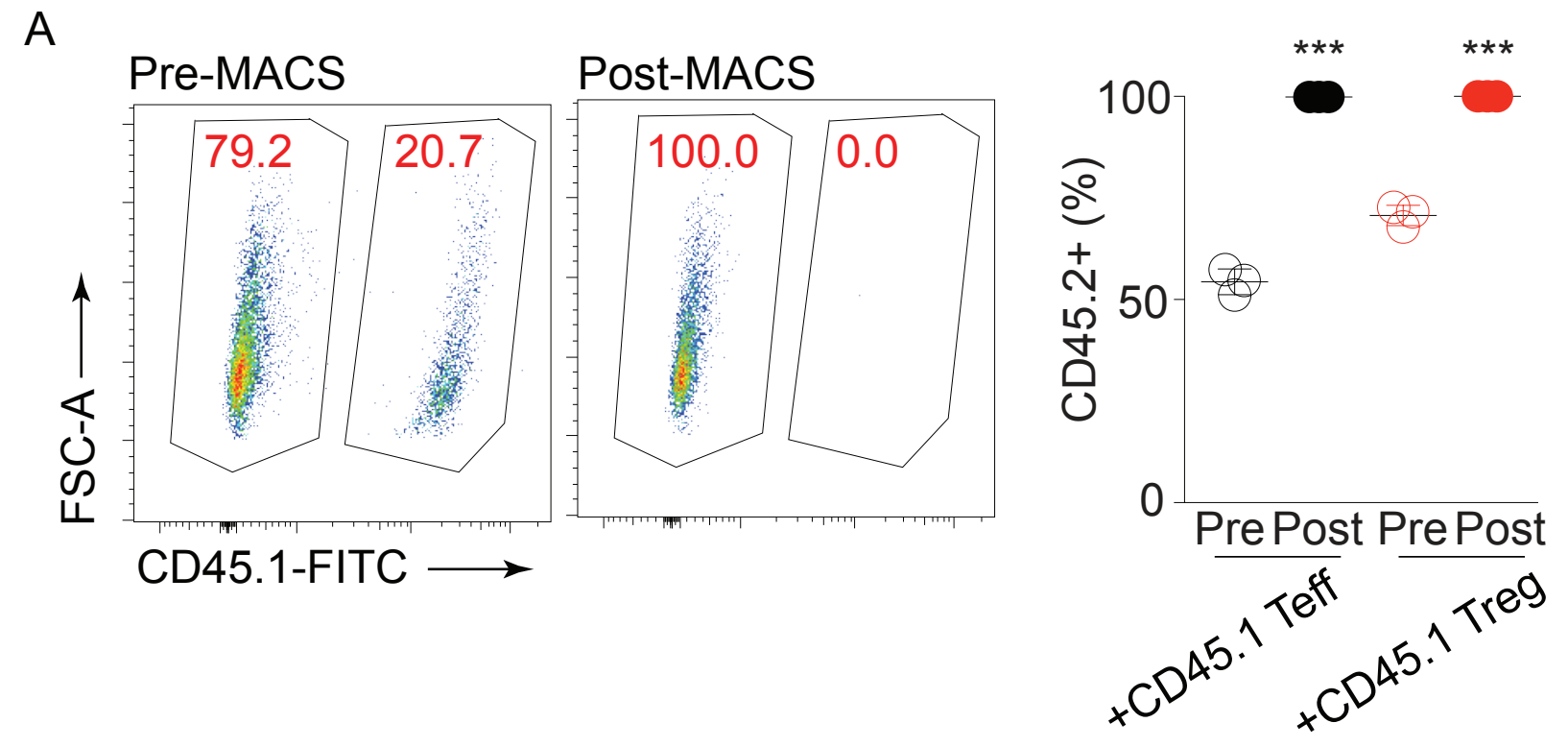

B

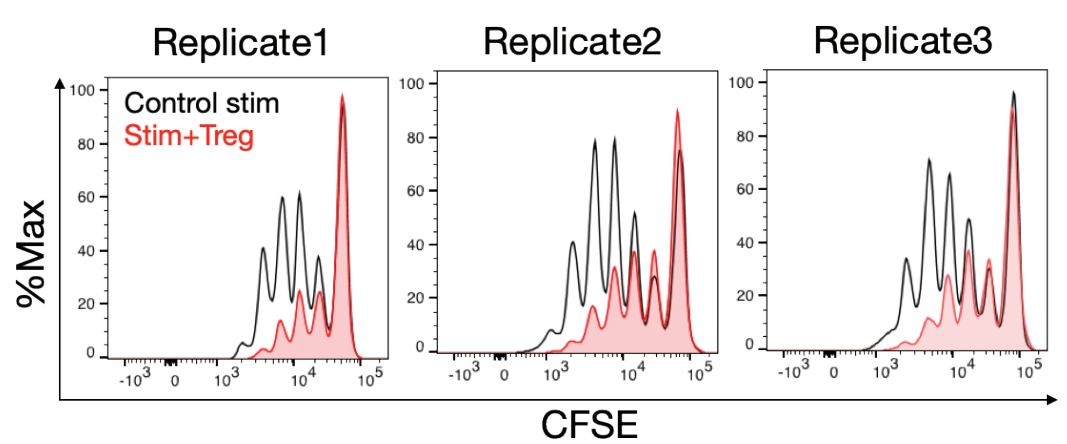

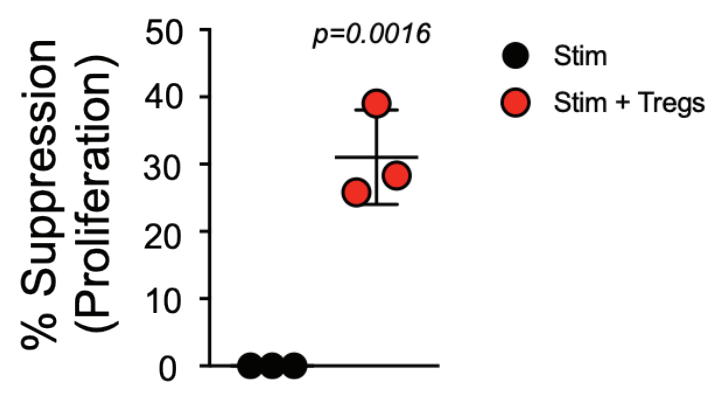


S Fig 4

A

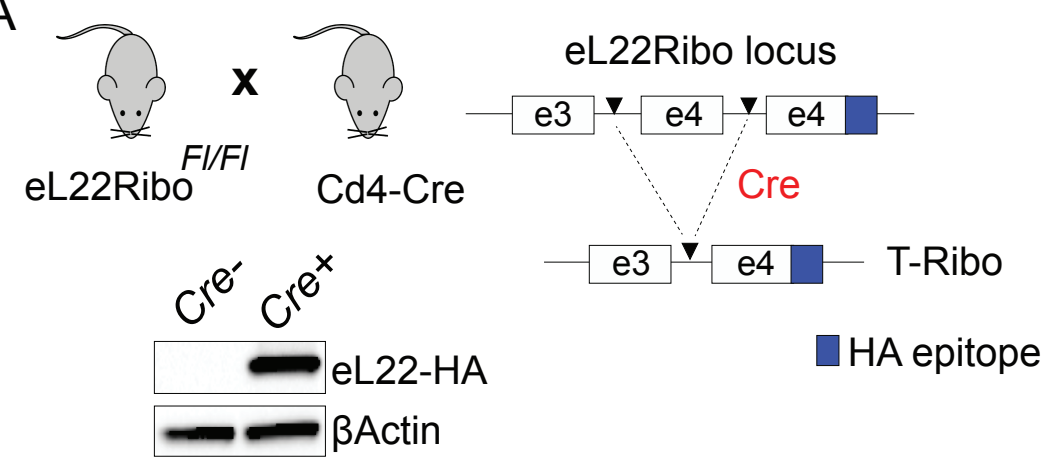

B

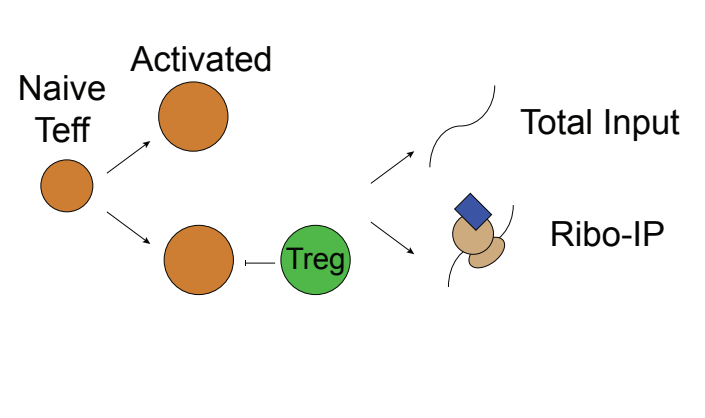

D

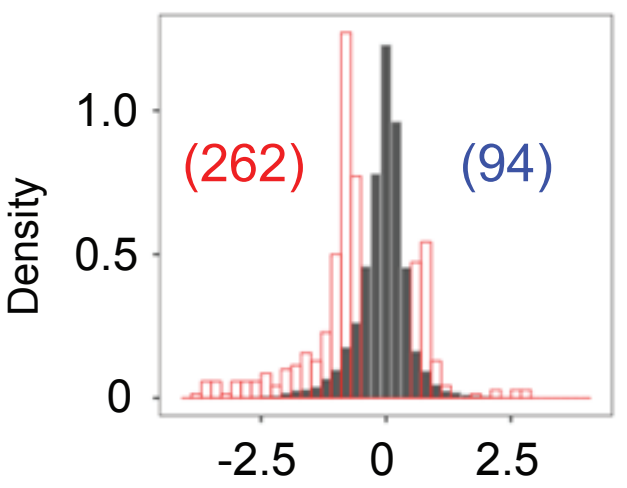

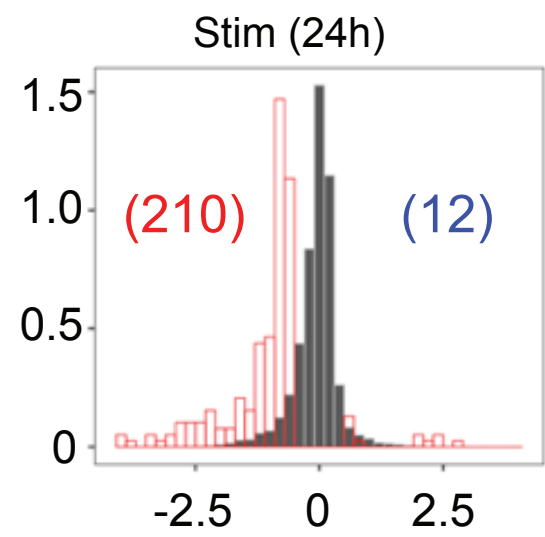

Ribosome Load Log2(Ribo-IP/Total Input)

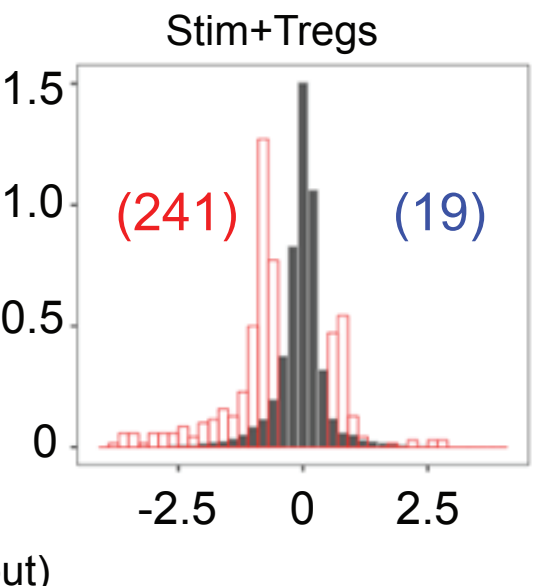

C

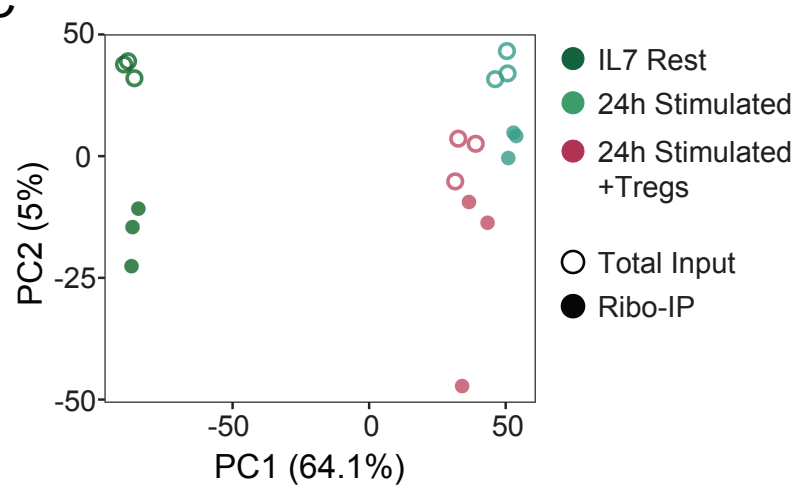

E

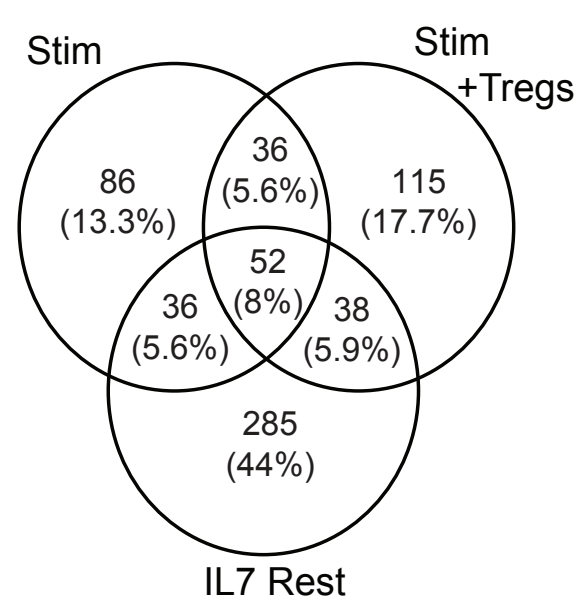

$\mathrm{F}$

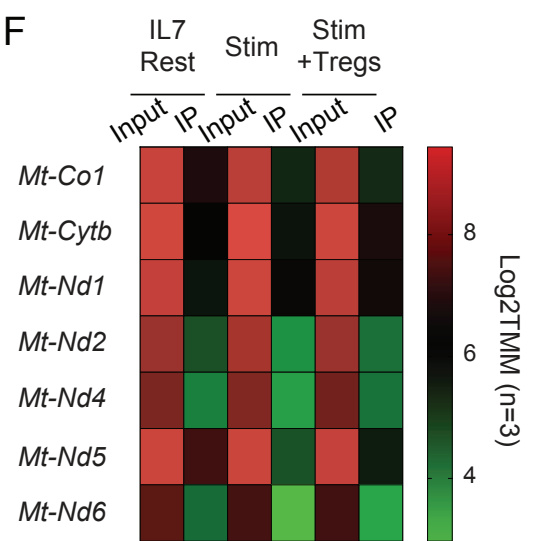

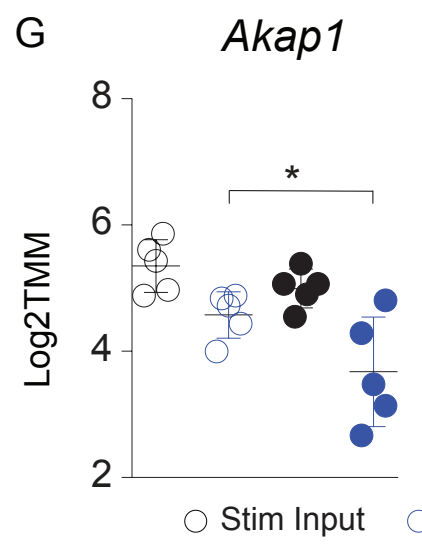

Btaf1

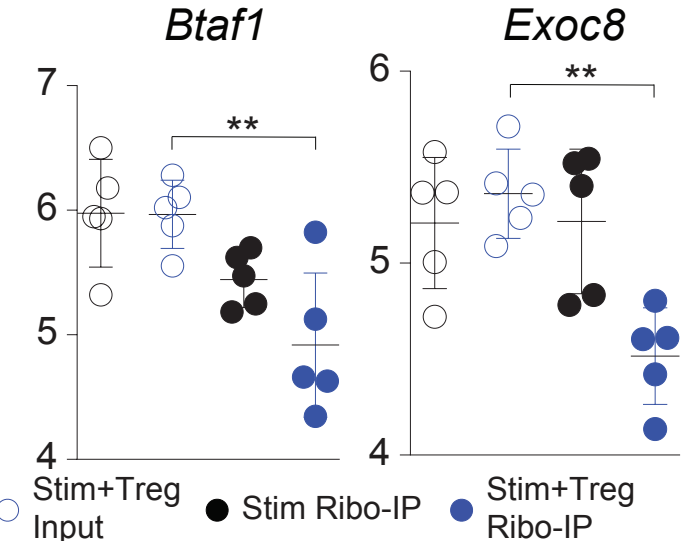


A

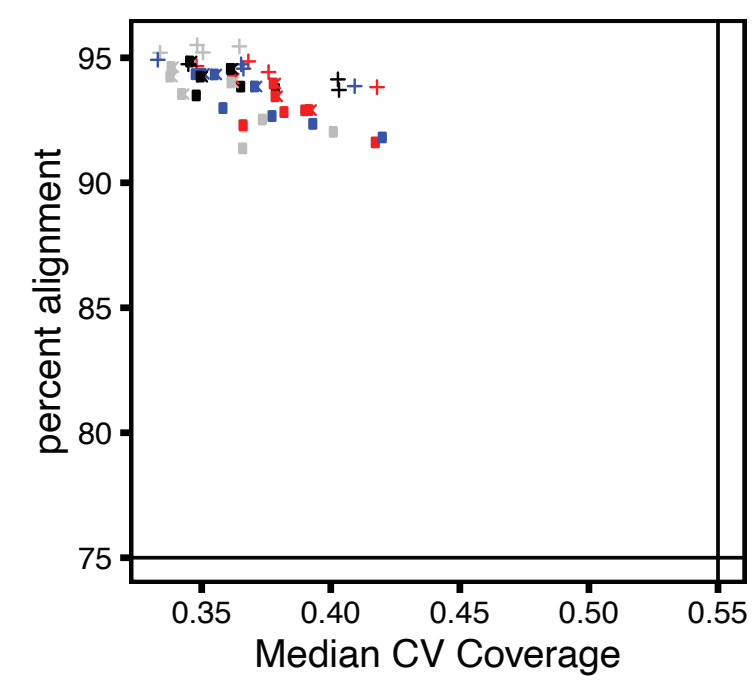

B

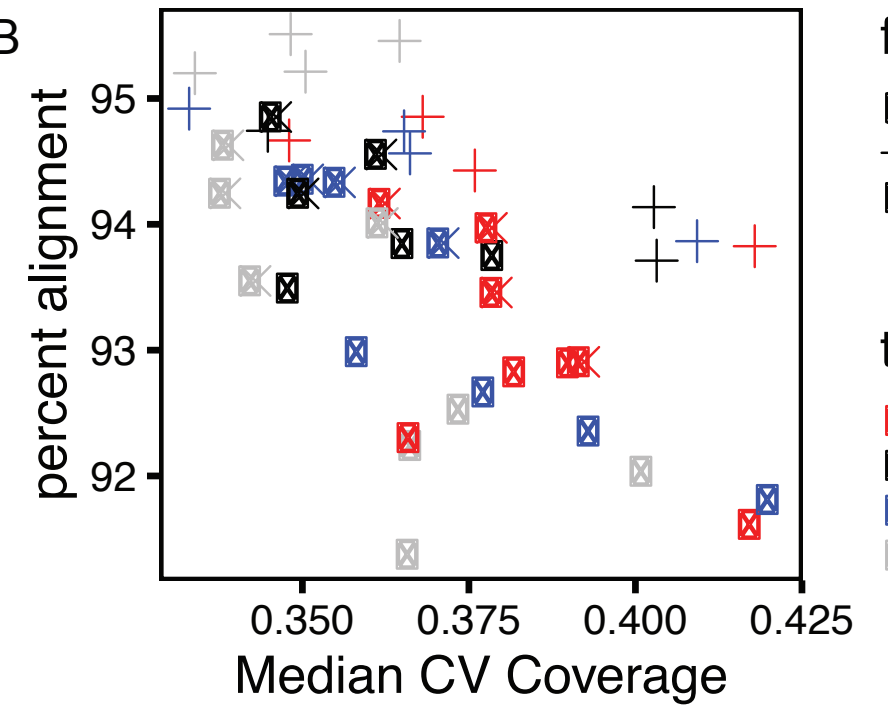

fraction

- Input

- SubPol

treatment

- Stim

- Stim + HHT

- Stim + Treg

Unstim

fraction

Input

+ Pol

\ubPol

treatment

Stim

Stim $+\mathrm{HHT}$

\ Stim + Treg

Unstim
C
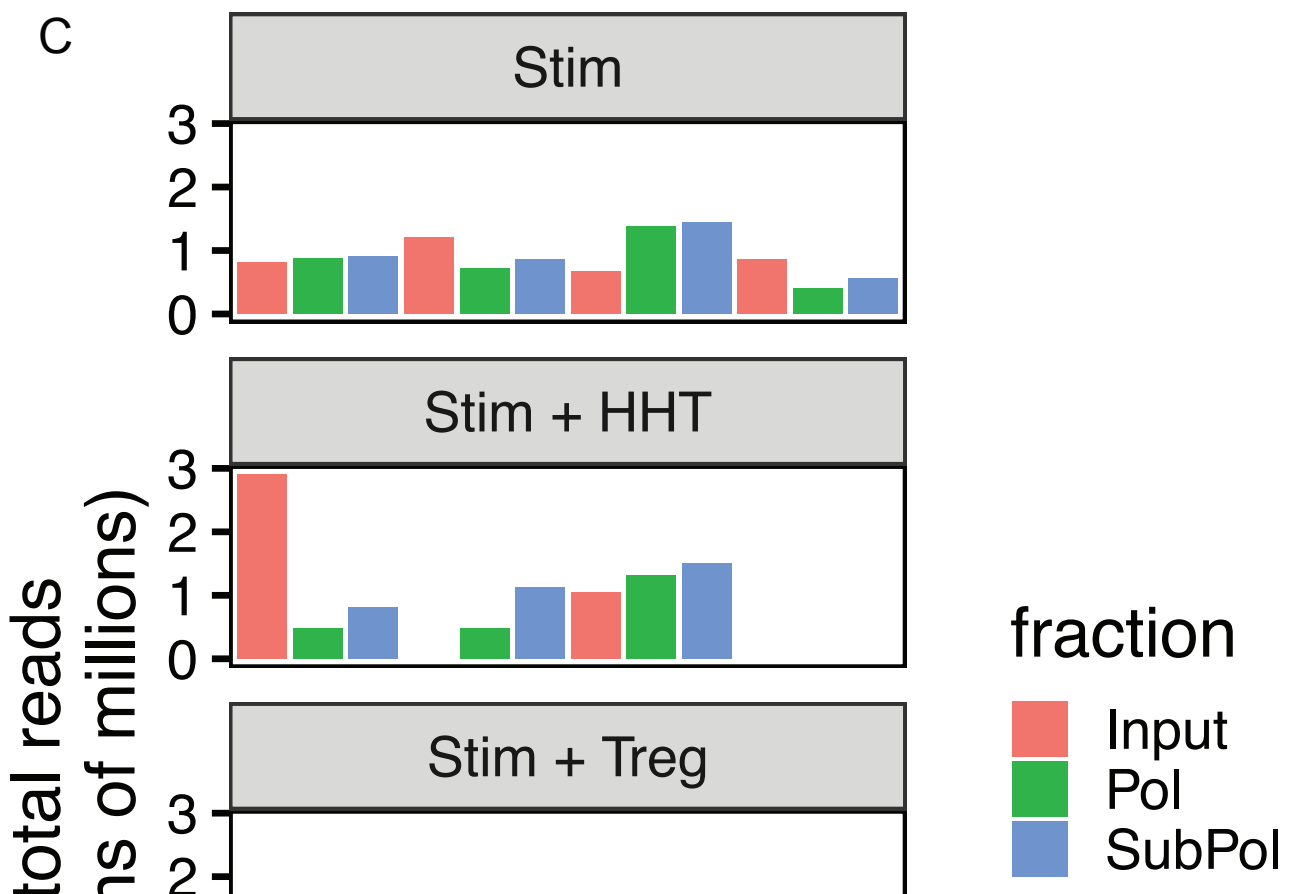
A
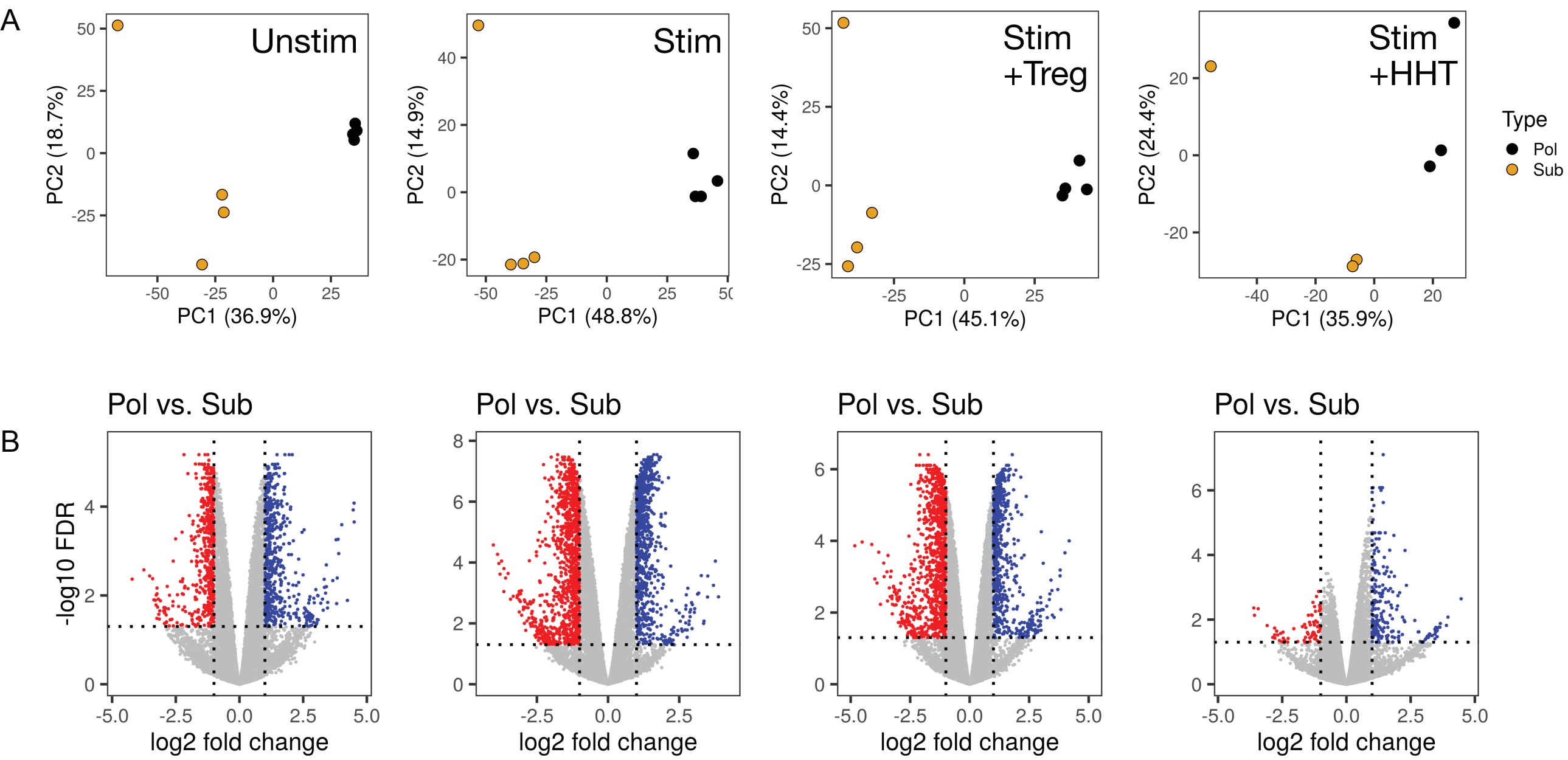
S Fig 7

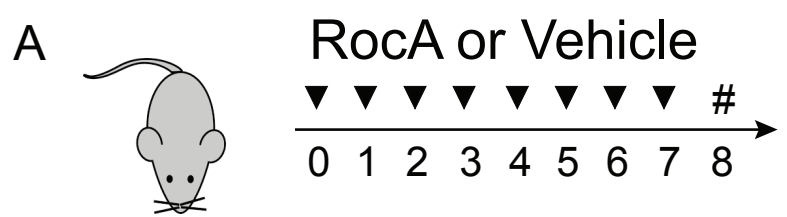

- DMSO • RocA $0.5 \mathrm{mg} / \mathrm{kg} \bullet$ RocA $1 \mathrm{mg} / \mathrm{kg}$

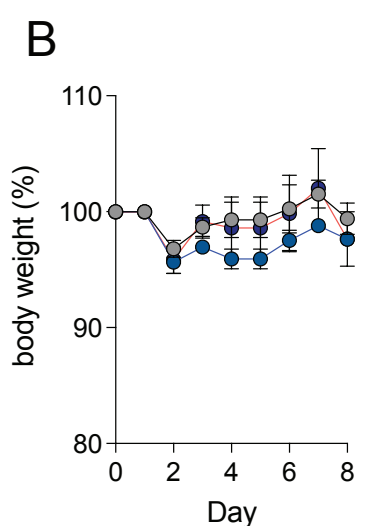

C Spleen
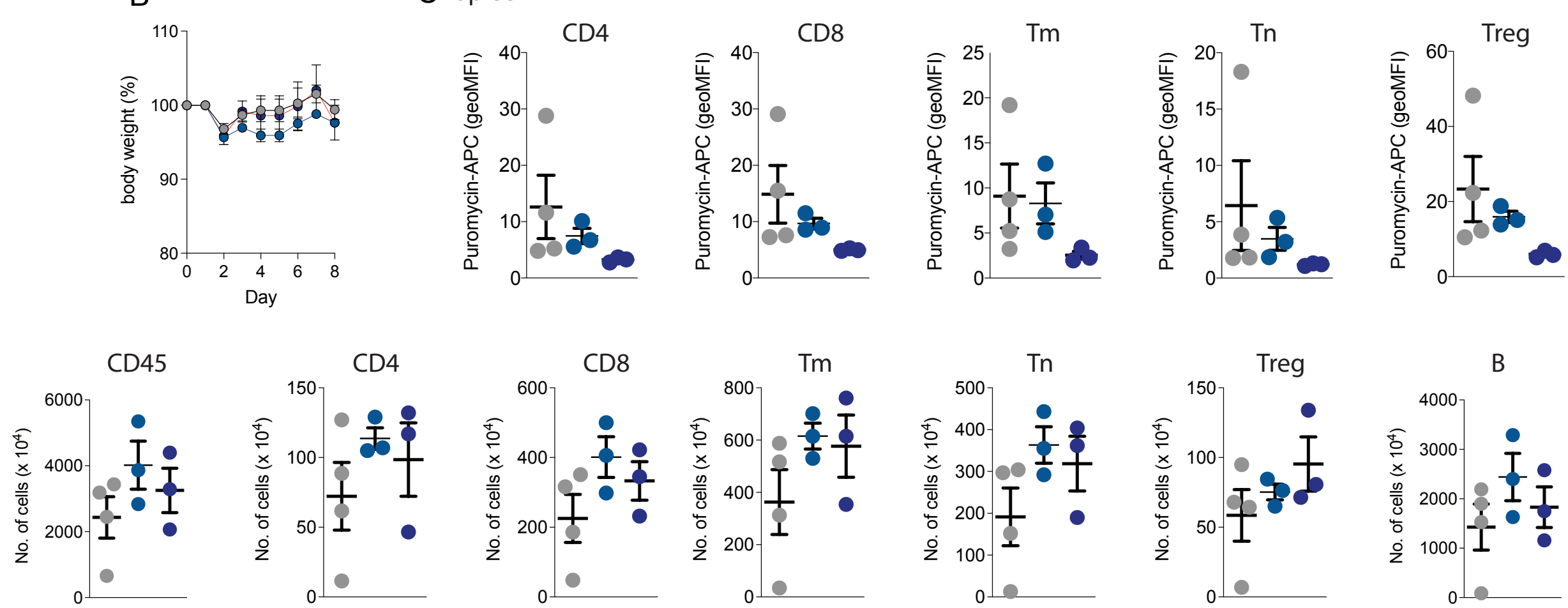

D Bone Marrow
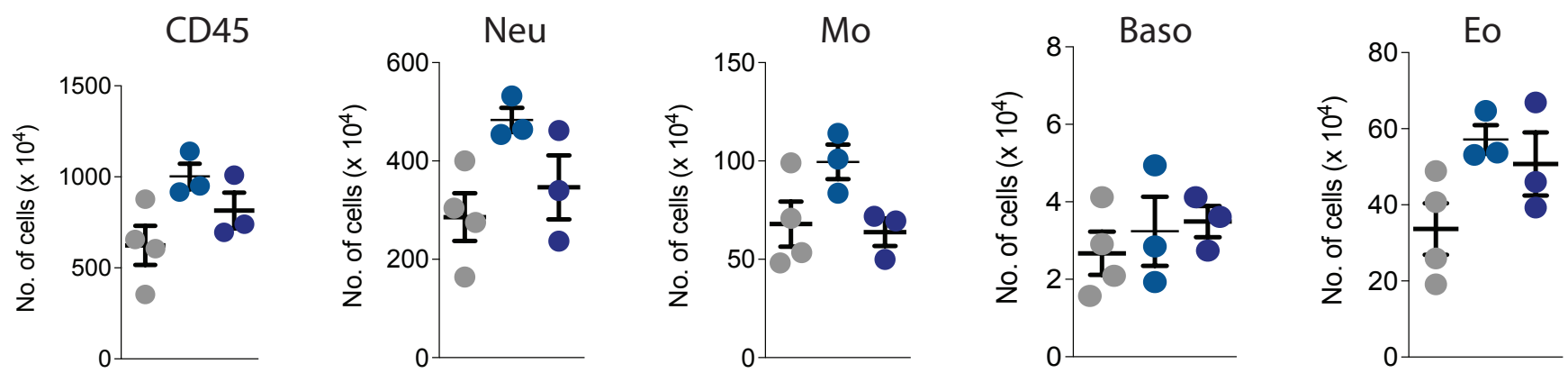


\section{Supplement text 1}

\section{Translatome capture by RiboTag.}

Mature CD4 Teff cells with an HA-epitope tag on the ribosomal large subunit protein L22 (eL22; known as RiboTag) were generated using Cd4-Cre mice ( $L 222^{\text {Ribofff }} C d 4 C r e$ : T-Ribo) where exon 4 of eL22 is substituted with an identical exon containing HA epitope sequences (Fig 2A). No obvious defects were observed in steady-state mature $\mathrm{T}$ cell frequencies in the spleen and lymph nodes with only a mild reduction in CD4+ T cell frequency (Fig S2A). Purified CD4 T cells from T-Ribo (Cd4-Cre+) expressed eL22-HA protein that co-sedimented with polysome fractions upon activation indicating normal ribosome incorporation (Fig S2B). Immunoprecipitation of eL22-HA (termed Ribo-IP) followed by mass spectrometry also identified most ribosomal proteins (RPs: 68/80) suggesting eL22-HA as a bona fide RP assembled as an intact ribosome (Fig S2C). Ribo-IP was highly efficient with complete depletion of eL22-HA with as little as $2 \mathrm{~h}$ of antibody incubation (Fig S2D).

The RiboTag system allowed profiling of both the steady-state mRNA levels and the ribosomebound mRNA levels from the same cytosolic lysate from CD4 Teff cells (Fig 2B). CD4 Teff cells from TRibo mice were stimulated alone or co-cultured with isolated Tregs and stimulated with anti-CD3/CD28 coated beads for $24 \mathrm{~h}$, a timepoint before the onset of proliferation. For resting conditions, we used CD4 T cells cultured in IL-7, which promotes survival but not activation, to match the timepoint for lysate preparation. Cytoplasmic lysates were prepared from pure CD4 Teffs after magnetic activated cell sorting (MACS) to remove either congenically marked CD4 Teff cells or Tregs where we obtained $>99.8 \%$ purity (Fig S3A). A small aliquot from the lysate (10\%) was taken for total input RNA extraction and Ribo-IP was performed on the remaining lysates to extract ribosome-bound mRNA. We also confirmed that Tregs faithfully suppressed CD4 Teff cell proliferation 3 days after stimulation compared to control stimulated Teff cells (Fig S3B). Ribosome-bound mRNA and total RNA input for each condition were subjected to RNA-seq. mRNA pulldown by Ribo-IP was highly specific as control activated CD4 Teff cells from Cre- mice ( $\mathrm{n}=2$ ) showed negligible traces of RNA based on Bioanalyzer analysis (Fig S3C). Principal component analysis (PCA) analysis separated the samples by the first component $(64.1 \%$ of variance) according to their activation status (IL7 rested vs activated) and suppressed CD4 Teff cells separated from activated CD4 Teff cells by the second component (5\% of variance) (Fig S4C). Importantly, total RNA input samples were clearly separated from their corresponding Ribo-IP samples by the second component indicating discrepancies in the total RNA input and its corresponding Ribo-IP mRNA. mRNAs of the mitochondrial DNA (mtDNA) are normally translated by mitoribosomes and not eL22 containing cytosolic ribosomes. In all conditions, we observed a significant de-enrichment of mitochondrial derived mRNAs in the Ribo-IP samples, internally validating that the Ribo-IP faithfully captures mRNAs only bound to the cytosolic ribosome (Fig S4F).

As total input samples were distinct from their corresponding Ribo-IP samples, we measured 'ribosome load' (RL) by calculating the ratio of Ribo-IP signal to its total RNA input signal. In all conditions, RL values were significantly biased towards the negative direction indicating the majority of mRNAs have less ribosome occupancy compared to their total input levels (Fig S4D). We attribute this bias to the fact that Ribo-IP signals represent mRNAs bound to all ribosomes, even including monosomes, thereby efficiently distinguishing free mRNAs from ribosome-bound mRNAs but not necessarily resolving polysome-bound mRNAs. One question arising from this is whether mRNAs with low RLs found in all conditions have low translation efficiencies due to their intrinsic properties in $\mathrm{T}$ cells or whether they are 
dynamically regulated depending on the stimuli. We found very low overlap $(<8 \%)$ between the differential RL mRNAs in all our conditions supporting the notion that the stimuli dictated the outcome of ribosome binding of a given mRNA in CD4 Teff cells (Fig S4E).

We assessed whether there were mRNAs with significantly lower RLs in the Treg suppressed Teff cells compared to the stimulated control. A distinct set of mRNAs including Akap1, Btaf1, and Exoc8 displayed lower RL only in suppressed Teff cells with no change in total RNA input levels (Fig SG). Suppressed mRNAs comprised protein coding genes not studied in the context of $\mathrm{T}$ cell activation, perhaps due to no apparent changes in the RNA level alone. A kinase anchor protein 1 (Akapl) and its family members have been extensively studied in the regulation of cAMP signaling by spatial and temporal regulation of the components in this pathway. However, no definitive role for this protein has been identified in the context of CD4 T cell activation. BTAF1 (Btaf1: TATA-binding protein-associated factor 172), a co-factor of B-TFIID has recently been shown to be a direct target of miR-132, a miRNA upregulated during CD4 T cell activation. In the context of CD4 T cell activation, BTAF1 has been shown to be a context-dependent activator of ribosomal protein gene transcription and forced expression of miR-132 results in global protein synthesis shutdown through BTAF1 silencing. The exocyst complex component 8 (Exoc8) is a member of the octameric exocyst complex involved in membrane trafficking as well as efficient cytokine secretion in activated T cells. In summary, the RiboTag approach revealed context dependent changes in gene expression beyond transcriptome changes and suggested an active regulation of ribosome dissociation of mRNAs in CD4 Teff cells by Tregs for the first time. We were able to identify mRNAs transcriptionally unaffected by activation in the presence of Tregs, but whose association with ribosomes was inhibited (Fig S4G). These data support a role for Treg-mediated regulation of mRNA translation. 
logFC AveExpr $\mathrm{t}$

ENSMUSG00000007850 ENSMUSG00000026003 ENSMUSG00000024097 ENSMUSG00000022858 ENSMUSG00000021218 ENSMUSG00000029817 ENSMUSG00000038299 ENSMUSG00000030934 ENSMUSG00000022186 ENSMUSG00000021428 ENSMUSG00000026234 ENSMUSG00000022300 ENSMUSG00000028790 ENSMUSG00000015837 ENSMUSG00000025190 ENSMUSG00000056851 ENSMUSG00000030560 ENSMUSG00000028330 ENSMUSG00000055762 ENSMUSG00000004789 ENSMUSG00000001525 ENSMUSG00000024360 ENSMUSG00000024975 ENSMUSG00000030884 ENSMUSG00000026260 ENSMUSG00000020361 ENSMUSG00000001016 ENSMUSG00000038510 ENSMUSG00000019179 ENSMUSG00000015672 $\begin{array}{lll}1.452705465 & 6.90514408 & 9.670263598\end{array}$ $\begin{array}{llll}0.98579119 & 7.62000787 & 8.217608565\end{array}$ $\begin{array}{llll}1.016468763 & 8.94969103 & 7.930151142\end{array}$ $\begin{array}{llll}1.009681492 & 6.41853076 & 7.467585597\end{array}$ $\begin{array}{lll}0.73561758 & 8.70454643 & 6.654320507\end{array}$ $\begin{array}{llll}1.557007491 & 6.66848205 & 6.331517803\end{array}$ $\begin{array}{llll}0.926291039 & 7.6128867 & 6.315681511\end{array}$ $\begin{array}{llll}0.840628651 & 8.36793726 & 6.312507651\end{array}$ $\begin{array}{llll}0.770088838 & 7.98865936 & 6.014881064\end{array}$ $\begin{array}{lll}1.23562732 & 7.14282707 & 5.8135716\end{array}$ $\begin{array}{lll}0.772953994 & 8.14352396 & 5.6639805\end{array}$ $\begin{array}{llll}0.584131698 & 8.47764336 & 5.646288592\end{array}$ $\begin{array}{lll}0.663325744 & 6.84075493 & 5.574478931\end{array}$ $\begin{array}{llll}0.737153452 & 8.10981975 & 5.570433668\end{array}$ $\begin{array}{lll}1.1512838 & 7.95376434 & 5.509762152\end{array}$ $\begin{array}{lll}0.675847891 & 7.15198435 & 5.496852376\end{array}$ $\begin{array}{lll}0.762388559 & 6.90098995 & 5.378450321\end{array}$ $\begin{array}{lll}0.677190353 & 7.95233827 & 5.374726368\end{array}$ $\begin{array}{llll}0.783824471 & 6.76796003 & 5.225935847\end{array}$ $0.716576197 \quad 8.06460372 \quad 5.202998056$ $\begin{array}{llll}0.727071908 & 9.14726002 & 5.179067786\end{array}$ $\begin{array}{llll}0.597908447 & 8.26516528 & 5.152472878\end{array}$ $\begin{array}{llll}0.754636722 & 7.96863182 & 5.123049193\end{array}$ $\begin{array}{llll}0.570868515 & 8.17005382 & 5.052642402\end{array}$ $\begin{array}{lll}0.544485297 & 8.40690526 & 5.02802777\end{array}$ $\begin{array}{llll}0.704062471 & 7.73062556 & 4.991224036\end{array}$ $\begin{array}{lll}0.608241392 & 8.13503831 & 4.98768173\end{array}$ $\begin{array}{lll}0.896138181 & 7.70566318 & 4.952115845\end{array}$ $\begin{array}{llll}0.635278652 & 9.11454946 & 4.911226748\end{array}$ $\begin{array}{lll}0.664658489 & 8.39904344 & 4.906823799\end{array}$
P.Value

8.37E-12

adj.P.Val

B 9.41E-08 $5.80 \mathrm{E}-10$ 3.26E-06 $1.38 \mathrm{E}-09$ $5.18 \mathrm{E}-06$ 5.69E-09 $7.16 \mathrm{E}-08$ $1.60 \mathrm{E}-05$ $1.98 \mathrm{E}-07 \quad 0.000236076$ $2.08 \mathrm{E}-07 \quad 0.000236076$ 2.10E-07 0.000236076 $5.38 \mathrm{E}-07 \quad 0.000549654$ $1.02 \mathrm{E}-06 \quad 0.00076197$ $1.63 \mathrm{E}-06 \quad 0.001019085$ 1.73E-06 0.001020983 2.17E-06 0.001173979 $2.19 \mathrm{E}-06 \quad 0.001173979$ $2.66 \mathrm{E}-06 \quad 0.001244116$ $2.77 \mathrm{E}-06 \quad 0.001244116$ 4.02E-06 0.001632896 4.07E-06 0.001632896 6.50E-06 0.002282407 $6.99 \mathrm{E}-06 \quad 0.00233642$ 7.53E-06 0.002417881 8.19E-06 0.002432597 $8.98 \mathrm{E}-06 \quad 0.002522347$ $1.12 \mathrm{E}-05 \quad 0.002995334$ $1.21 \mathrm{E}-05 \quad 0.003160004$ $1.36 \mathrm{E}-05 \quad 0.003351167$ 1.37E-05 0.003351167 $1.53 \mathrm{E}-05 \quad 0.003665291$ $1.74 \mathrm{E}-05 \quad 0.003890526$ $1.77 \mathrm{E}-05 \quad 0.003890526$ 15.8065461 Hnrnph1 12.56564947 Acadl 11.868448 Srsf7 10.02167782 Tra2b 8.073005656 Gdi2 6.945128554 Tra2a $7.043274241 \mathrm{Wdr} 36$ 7.039582708 Oat 6.155690568 Oxct1 5.551561545 Riok1 $5.09298276 \mathrm{Ncl}$ 5.015065136 Dcaf13 4.823981214 Khdrbs1 4.793024859 Sqstm1 4.616939093 Got1 4.607194401 Pcbp2 $4.259276828 \mathrm{Ctsc}$ 4.221588482 Ncbp1 3.777295542 Eef1d 3.705666202 Dlst 3.598111261 Tubb5 3.525963425 Etf1 3.446657309 Pdcd4 3.246696042 Uqcrc2 3.160411278 Ndufa10 3.109258072 Hspa4 3.068797488 IIf2 3.008031677 Rpf2 2.787317165 Mdh2 2.783312229 Mrpl32 
ENSMUSG00000022336 ENSMUSG00000060373 ENSMUSG00000079111 ENSMUSG00000031715 ENSMUSG00000053565 ENSMUSG00000021832 ENSMUSG00000020708 ENSMUSG00000005625 ENSMUSG00000064023 ENSMUSG00000025794 ENSMUSG00000035202 ENSMUSG00000001783 ENSMUSG00000047514 ENSMUSG00000016541 ENSMUSG00000001767 ENSMUSG00000020612 ENSMUSG00000018326 ENSMUSG00000001962 ENSMUSG00000001380 ENSMUSG00000038462 ENSMUSG00000040028 ENSMUSG00000033793 ENSMUSG00000055044 ENSMUSG00000037916 ENSMUSG00000025066 ENSMUSG00000027433 ENSMUSG00000007739 ENSMUSG00000020069 ENSMUSG00000027170 ENSMUSG00000041459 ENSMUSG00000063884 $\begin{array}{lll}0.617783361 & 9.09580155 & 4.86757586\end{array}$ $\begin{array}{lll}0.525744431 & 8.24858231 & 4.814231626\end{array}$ $\begin{array}{llll}0.813996178 & 7.79214496 & 4.800921921\end{array}$ $\begin{array}{llll}0.897340719 & 5.96874637 & 4.791857973\end{array}$ $\begin{array}{llll}0.530976072 & 9.6074959 & 4.750815209\end{array}$ $\begin{array}{llll}0.556180466 & 8.76452888 & 4.742774231\end{array}$ $\begin{array}{llll}0.5046479 & 9.14988387 & 4.675706777\end{array}$ $\begin{array}{llll}0.500966439 & 8.09176807 & 4.654180928\end{array}$ $\begin{array}{llll}0.506522511 & 7.68403189 & 4.617437191\end{array}$ $\begin{array}{lll}0.641642326 & 8.1989659 & 4.593984143\end{array}$ $\begin{array}{llll}1.057772713 & 6.50116884 & 4.584950685\end{array}$ $\begin{array}{lll}0.478244045 & 8.3917028 & 4.57546983\end{array}$ $\begin{array}{llll}0.602442179 & 7.24014323 & 4.572611467\end{array}$ $\begin{array}{llll}0.530998893 & 8.58354601 & 4.541460749\end{array}$ $\begin{array}{lll}0.885148311 & 6.217693 & 4.530413032\end{array}$ $\begin{array}{llll}0.504320895 & 8.53295621 & 4.523038915\end{array}$ $\begin{array}{llll}0.484013903 & 8.67153626 & 4.505671374\end{array}$ $\begin{array}{llll}0.685877503 & 7.10377649 & 4.496997486\end{array}$ $\begin{array}{llll}0.54583261 & 8.38689755 & 4.482592729\end{array}$ $\begin{array}{lll}0.497613447 & 9.22116356 & 4.473856674\end{array}$ $\begin{array}{lll}0.602477276 & 7.11689312 & 4.451429784\end{array}$ $\begin{array}{lll}0.674124483 & 7.83084463 & 4.431867601\end{array}$ $\begin{array}{llll}0.56245614 & 6.53590316 & 4.426887132\end{array}$ $\begin{array}{lll}0.568827616 & 7.9625517 & 4.35217982\end{array}$ $\begin{array}{llll}0.522301986 & 8.80876721 & 4.302474037\end{array}$ $\begin{array}{llll}0.577157298 & 8.13001897 & 4.295863033\end{array}$ $\begin{array}{llll}0.461178408 & 8.33419346 & 4.287350538\end{array}$ $\begin{array}{llll}0.895767788 & 6.04132415 & 4.278635442\end{array}$ $\begin{array}{lll}0.588901934 & 7.87588804 & 4.274984634\end{array}$ $\begin{array}{lll}0.775827289 & 6.30279505 & 4.261257252\end{array}$ $\begin{array}{llll}0.664128116 & 7.79591832 & 4.239784593\end{array}$
$2.00 \mathrm{E}-05 \quad 0.004152356$

$2.36 \mathrm{E}-05 \quad 0.004812786$

$2.46 \mathrm{E}-05 \quad 0.004926427$

$2.53 \mathrm{E}-05 \quad 0.004978191$

2.87E-05 0.005462669

$2.94 \mathrm{E}-05 \quad 0.00550721$

3.62E-05 0.005980462

3.87E-05 0.006289241

4.33E-05 0.00676142

4.66E-05 0.007071752

4.79E-05 0.00716537

4.93E-05 0.00716537

4.97E-05 0.00716537

$5.47 \mathrm{E}-05 \quad 0.007688453$

$5.66 \mathrm{E}-05 \quad 0.007855629$

$5.79 \mathrm{E}-05 \quad 0.007937511$

$6.11 E-05 \quad 0.008172624$

$6.27 \mathrm{E}-05 \quad 0.008197717$

6.56E-05 0.008469211

$6.73 \mathrm{E}-05 \quad 0.008600014$

7.21E-05 0.009084323

$7.66 \mathrm{E}-05 \quad 0.009353373$

$7.78 \mathrm{E}-05 \quad 0.009394645$

9.76E-05 0.01119509

$0.000113544 \quad 0.012638069$

0.0001158420 .012638069

$0.00011887 \quad 0.012843635$

0.0001220490 .012856671

$0.000123406 \quad 0.012856671$

$0.00012864 \quad 0.013141148$

$\begin{array}{lll}0.000137266 & 0.013555147\end{array}$
2.661295677 Eif3e 2.506596729 Hnrnpc 2.539486047 Kdelr2 2.450713856 Smarca5 2.341480088 Eif3k 2.283146083 Psmc6 2.087934611 Psmc5 2.054628773 Psmd4 1.955555418 Klk8 $1.87863647 \mathrm{Rpl} 14$ 1.944816052 Lars2 1.815113226 Rtcb 1.850537263 Tspyl1 1.700527025 Atxn10 1.801617167 Crnkl1 1.640371706 Prkar1a 1.583258024 Ywhab 1.679578144 Fam50a 1.559982406 Hars 1.504785068 Uqcrfs1 1.574343171 Elavl1 1.43361175 Atp6v1h 1.523037486 Pdlim1 1.227028901 Ndufv1 $0.991126782 \mathrm{Sfr} 1$ 1.018555223 Xrn2 0.974744677 Cct4 1.095162808 Hnrnph3 0.991110731 Eif3m 1.045687306 Tardbp 0.909081998 Ptcd3 
ENSMUSG00000005846 ENSMUSG00000042590 ENSMUSG00000047879 ENSMUSG00000039828 ENSMUSG00000007564 ENSMUSG00000071172 ENSMUSG00000022024 ENSMUSG00000026377 ENSMUSG00000026575 ENSMUSG00000031701 ENSMUSG00000022312 ENSMUSG00000004018 ENSMUSG00000052253 ENSMUSG00000029169 ENSMUSG00000078920 ENSMUSG00000070697 ENSMUSG00000031731 ENSMUSG00000025742 ENSMUSG00000031320 ENSMUSG00000025337 ENSMUSG00000014195 ENSMUSG00000007891 ENSMUSG00000068882 ENSMUSG00000029422 ENSMUSG00000018446 ENSMUSG00000025287 ENSMUSG00000031708 ENSMUSG00000031672 ENSMUSG00000027455 ENSMUSG00000026753 ENSMUSG00000003970
0.4734239748 .89081698 1.2066318516 .50448663 $0.67389615 \quad 6.82130773$

4.239178221 4.220945002 4.21941351 0.7072947987 .16843271

0.5019235878 .42762787

0.54198487 .24453192 $0.45005592 \quad 8.12982002$ 0.4687030258 .51671519 1.0757099347 .12446427 0.4086528278 .53106947 210507418 4.198845115 4.19779481 4.187810207 4.185823182 4.174948861 4.16387302 $\begin{array}{llll}0.477200682 & 9.18841857 & 4.133304815\end{array}$ $\begin{array}{llll}0.816469135 & 6.17763262 & 4.131692294\end{array}$ $\begin{array}{lll}0.539580237 & 7.33972185 & 4.099393924\end{array}$ $\begin{array}{lll}0.506186457 & 8.44678662 & 4.097710813\end{array}$ $\begin{array}{lll}0.516677536 & 8.94102912 & 4.074287279\end{array}$ $\begin{array}{lll}0.551595722 & 8.307165 & 4.021746888\end{array}$ $\begin{array}{llll}0.982814777 & 6.14568901 & 4.021676386\end{array}$ $\begin{array}{llll}0.550941235 & 7.323234 & 4.001134758\end{array}$ $\begin{array}{lll}0.723100928 & 6.56641085 & 3.989840437\end{array}$ $\begin{array}{lll}0.533188669 & 7.62183042 & 3.985034296\end{array}$ $\begin{array}{lll}0.434839468 & 7.06664405 & 3.958312697\end{array}$ $\begin{array}{lll}0.461934514 & 7.70308641 & 3.924970179\end{array}$ $\begin{array}{llll}0.531333297 & 8.31899707 & 3.895829127\end{array}$ $\begin{array}{lll}0.627342641 & 6.11913237 & 3.87280679\end{array}$ $\begin{array}{lll}0.628272621 & 7.91276715 & 3.870998635\end{array}$ $\begin{array}{lll}0.527510232 & 7.56674985 & 3.849723927\end{array}$ $\begin{array}{lll}0.422546449 & 9.09853232 & 3.845936718\end{array}$ $\begin{array}{lll}0.650158104 & 6.91057442 & 3.845207005\end{array}$ $\begin{array}{lll}0.559335682 & 6.41766652 & 3.827564914\end{array}$ $0.5201133497 .37478302 \quad 3.804452891$ $\begin{array}{lll}0.599233238 & 10.210691 & 3.800252536\end{array}$
0.000137518 0.000145299 0.000145972 0.000149945 0.000155309 0.000155801 0.000160557 0.00016152 0.000166892 0.000172543 0.000189127 0.000190044 0.000209347 0.000210404 0.000225662 0.000263915 0.00026397 0.000280588 0.000290154 0.00029432 0.000318568 0.00035156 0.000383095 0.000409935

0.000412118 0.000438674 0.00044357 0.00044452 0.000468095 0.000500818 0.000506999
0.013555147 0.014019529 0.014019529 0.014159133 0.014350323 0.014350323 0.014637099 0.014637099 0.014715343 0.014800506 0.01536346 0.01536346 0.016533629 0.016533629 0.017488013 0.019091048 0.019091048 0.019955472 0.020251305 0.020298011 0.021564771 0.022967898 0.024184473 0.025032287 0.025032287 0.025747882 0.025747882 0.025747882 0.026597382 0.027632597 0.027656034
0.809032869 Rsl1d1 0.956754485 Ipo11 0.950243337 Usp14 0.865313229 Wdr70 0.707283802 Ppp2r1a 0.833486924 Srsf3 0.700766906 Sugt1 0.678862559 Nifk $0.831284128 \mathrm{Nme} 7$ 0.597479788 Dnaja2 0.527804841 Eif3h 0.682322676 Fancl 0.500557885 Zfp622 0.41891431 Dhx15 0.33953031 Ifi47 0.224187012 Utp3 0.423699378 Ap1g1 0.278575898 Prps 2 0.334738166 Rps4x 0.167755405 Sbds 0.174348408 Dnajc7 -0.032425437 Ctsd

-0.121199477 Ssb 0.026210616 Rsrc2 -0.074395047 C1qbp -0.135480259 Acot9 $-0.293453334 \mathrm{Tecr}$ -0.04120348 Got2 $-0.087045321 \mathrm{Nsfl} 1 \mathrm{c}$ -0.289614225 Ppp6c $-0.329235345 \mathrm{Rpl} 8$ 
ENSMUSG00000001774 ENSMUSG00000043931 ENSMUSG00000008333 ENSMUSG00000030798 ENSMUSG00000003429 ENSMUSG00000037022 ENSMUSG00000020664 ENSMUSG00000028187 ENSMUSG00000026727 ENSMUSG00000062203 ENSMUSG00000091649 ENSMUSG00000020372 ENSMUSG00000041645 ENSMUSG00000022247 ENSMUSG00000014769 ENSMUSG00000059208 ENSMUSG00000029486 ENSMUSG00000041028 ENSMUSG00000022052 ENSMUSG00000027248 ENSMUSG00000057113 ENSMUSG00000041278 ENSMUSG00000003421 ENSMUSG00000095567 ENSMUSG00000022905 ENSMUSG00000032458 ENSMUSG00000062006 ENSMUSG00000051695 ENSMUSG00000024191 ENSMUSG00000036427 ENSMUSG00000028249
0.655274518 .06265364 0.4768254878 .71621878 0.6434230416 .8193216 0.5058441117 .22041593 0.6585237219 .21718406 1.4292612154 .13198304 0.7982232527 .59507404 0.5139830668 .55795011 0.6546362215 .55691335 0.6363673556 .95600969 $0.482749213 \quad 8.37218129$ $0.638344526 \quad 8.21325616$ $0.495840593 \quad 8.15595142$ 0.5418781537 .88262391 0.4855364299 .45083188 0.4532161698 .22818485 0.8584198395 .82399622 0.5105776628 .77446311 0.5075416227 .47163506 0.4233805118 .8264423 0.6426322398 .1077045 $0.422910803 \quad 7.757378$ 0.4926635097 .20372668 0.4000513138 .5687401 0.8982844126 .01877168 $\begin{array}{lll}0.519633323 & 7.79657371 & 3.560170523\end{array}$ $\begin{array}{lll}0.611162599 & 7.55924468 & 3.559794549\end{array}$ $0.438854898 .98156134 \quad 3.551553424$ $\begin{array}{llll}1.231564931 & 4.85132548 & 3.551119529\end{array}$ $\begin{array}{lll}0.488283041 & 7.73501084 & 3.550350847\end{array}$ $\begin{array}{lll}0.418889314 & 7.21901382 & 3.547862045\end{array}$
3.78931761

3.780986936

3.777980133

3.775450918

3.740937031

3.739577911

3.738698439

3.737498087

3.736676631

3.734183041

3.718086143

3.712550282

3.704968873

3.701635867

3.684258023

3.675459368

3.661384441

3.651036533

3.64310802

3.640381289

3.632665529

3.606305008

3.597822764

3.581665818

\section{0}

0.00101365

0.001014736

0.001038842

0.001040126

0.001042405

0.001049817
0.028414776 0.028819061 0.028819061 0.028888926 0.030468068 0.030468068 0.030468068 0.030468068 0.030468068 0.030553536 0.031593806 0.031964835 0.032253288 0.032426592 0.033599346 0.034211775 0.034928985 0.035548222 0.036058084 0.036070336 0.036570446 0.037514775 0.037606865 0.038955322 0.039289927 0.040434724 0.040434724 0.040956315 0.040956315 0.040956315 0.041103805
-0.419480357 Chordc1 -0.477232676 Gimap7 -0.238834585 Snrpb2 $-0.387825543 \mathrm{Cd} 37$ -0.545751119 Rps11 $-0.483800356 \mathrm{Mmaa}$ -0.452200297 Dld -0.598444726 Rpf1 -0.325539063 Rsu1 -0.330599182 Gspt1 -0.567019808 Phf11b -0.618610098 Rack1 -0.660628582 Ddx24 -0.614110386 Brix1 -0.712381771 Psmb1 -0.715940759 Hnrnpm $-0.533580228 \mathrm{Mrpl} 1$ -0.832866627 Ghitm -0.756040941 Ppp2r2a -0.860121999 Pdia3 $-0.797050125 \mathrm{Npm} 1$ -0.872439897 Ttc1 -0.788996388 Nosip -1.005111033 Noc2l -0.753499121 Kpna1 -0.938806591 Copb2 -0.915345781 Rpl34 -1.099284823 Pcbp1 -1.051371456 Bnip1 -0.958039569 Gpi1 -0.988117964 Sdcbp 
ENSMUSG00000037204 ENSMUSG00000018189 ENSMUSG00000036693 ENSMUSG00000052146 ENSMUSG00000011114 ENSMUSG00000025651 ENSMUSG00000004771 ENSMUSG00000027374 ENSMUSG00000054079 ENSMUSG00000032096 ENSMUSG00000021282 ENSMUSG00000024188 ENSMUSG00000031807 ENSMUSG00000016921 ENSMUSG00000022827 ENSMUSG00000024404 ENSMUSG00000039356 ENSMUSG00000025525 ENSMUSG00000063457 ENSMUSG00000033161 ENSMUSG00000029328 ENSMUSG00000005683 ENSMUSG00000031304 ENSMUSG00000049760 ENSMUSG00000020589 ENSMUSG00000024539
0.4634948818 .06022019

0.4804867138 .46620946

$0.776914837 \quad 7.21014124$

0.6806896925 .98588746

0.4088958147 .11749723

0.511454949 .49403245

0.4177114937 .26062015

0.8413449295 .13205109

0.4821145427 .17985991

0.4485716947 .07916683

0.3208862418 .38745085

$0.821053708 \quad 5.91539543$

0.4587962796 .80981096

0.3896368848 .15231783

1.0858708044 .60698662

$0.509165188 \quad 7.32127404$

0.4575600197 .98022237

0.6583326596 .21572746

$0.470968762 \quad 8.926114$

0.7080566137 .1588568

$0.522764503 \quad 7.30768527$

0.4042139318 .58553601

$0.523071467 \quad 8.28879398$

$0.384488856 \quad 8.80461198$

0.6802243885 .84157261

0.4129274367 .60766941
3.54522422

3.541091168

3.531528157

3.530306219

3.526776563

3.514313062

3.511564722

3.510882684

3.5045578

3.497326881

3.494164902

3.492659102

3.475799805

3.473165879

3.472873848

3.469466179

3.456574676

3.455796573

3.447239647

3.444997465

3.440996754

3.436082796

3.432188177

3.418395329

3.411387322

3.410523107
0.001057728

0.001070238

0.001099732

0.001103557

0.001114676

0.001154807

0.001163841

0.001166093

0.001187182

0.001211739

0.001222631

0.001227851

0.00128777

0.001297382

0.001298451

0.001310998

0.001359522

0.001362505

0.001395729

0.001404562

0.001420457

0.001440215

0.001456062

0.001513536

0.001543564

0.001547306
$0.041269746-1.098487153$ Atg101 $0.041570649-1.068428469$ Uchl5

$0.041894151-0.923471344$ Nop14

$0.041894151-0.833319137$ Rps10

$0.042173792-0.987450098$ Tbrg 1

$0.043111506-1.173488165$ Uqcrc1

$0.043245513-1.060006004$ Rab11a

$0.043245513-0.97077027$ Mrps5

$0.043453942-1.019329602$ Utp18

$0.04406575-1.043523036$ Arcn1

$0.044318397-1.247051779$ Eif5

$0.04436449-0.927425943$ Luc7l

$0.045648823-1.008804009$ Pgls

$0.045738865-1.234988006$ Srsf6

$0.045738865-1.185214393$ Rabl3

$0.045905955-1.209944962$ Riok3

$0.046818517-1.224704706$ Exosc2

$0.046818517-1.011523416$ Apool

$0.047240384-1.369942805$ Rps15

$0.047254692-1.195988141$ Atp1a1

$0.047526889-1.185691314$ Hnrnpdl

$0.04773952-1.390395751$ Cs

$0.047981716-1.402642687$ II $2 \mathrm{rg}$

$0.049154927-1.446809412$ 2410015M20Rik

$0.049962878-1.139370591$ Fam49a

$0.049962878-1.362275157$ Ptpn2 
Supplementary Table 2: Reagent identifiers

\begin{tabular}{|c|c|c|}
\hline REAGENT or RESOURCE & SOURCE & IDENTIFIER \\
\hline \multicolumn{3}{|l|}{ Antibodies } \\
\hline $\begin{array}{l}\text { Anti-Puromycin, clone 12D10, AlexaFluor647 } \\
\text { Conjugate }\end{array}$ & EMD Milipore & MABE343-AF647 \\
\hline Anti-HA tag antibody-ChIP Grade & Abcam & Ab9110 \\
\hline S6 Ribosomal Protein (5G10) Rabbit antibody & $\begin{array}{l}\text { Cell Signaling } \\
\text { Technology }\end{array}$ & $2217 \mathrm{~L}$ \\
\hline CD4-BV421 (GK1.5) & BioLegend & 100438 \\
\hline CD62L-PE (MEL-14) & BioLegend & 104408 \\
\hline CD45.1-FITC (A20) & BioLegend & 110706 \\
\hline Alpha-Tubulin & $\begin{array}{l}\text { Cell Signaling } \\
\text { Technology }\end{array}$ & \\
\hline Anti-IL-10R, clone 1B1.3A & BioXcell & BE0050 \\
\hline Anti-IFNg, XMG1.2, PerCP/Cyanine5.5 conjugate & Biolegend & 505822 \\
\hline Anti-IL-17A, TC11-18H10.1, PE conjugate & Biolegend & 506904 \\
\hline Anti-human IFN-g, 4S.B3,APC conjugate & Biolegend & 502512 \\
\hline Anti-IL-13, JES10-5A2, PE-conjugate & Biolegend & 501903 \\
\hline Anti-IL-2, JSE6-1A12 & Biolegend & 503706 \\
\hline Anti-IL-2R, 3C7 & biolegend & 101926 \\
\hline \multicolumn{3}{|l|}{ Biological samples } \\
\hline Human PBMC & $\begin{array}{l}\text { Benaroya Research } \\
\text { Institute Biorepository }\end{array}$ & \\
\hline \multicolumn{3}{|l|}{ Chemicals, peptides, and recombinant proteins } \\
\hline Cycloheximide, ready-solution & Sigma & C4859-1ML \\
\hline Rocaglamide (Roc-A) & MedChemExpress & HY-19356 \\
\hline Recombinant Human IL-2 (carrier-free) & BioLegend & 589106 \\
\hline Recombinant Mouse TGFb1 (carrier-free) & BioLegend & 763104 \\
\hline Puromycin ready-made solution & Sigma & P9620-10ML \\
\hline Tacrolimus (FK506) & MedChemExpress & HY-13756 \\
\hline Sapanisertib (MLN0128) & MedChemExpress & HY-13328 \\
\hline CellTrace CFSE Cell Proliferation Kit & ThermoFisher & C34554 \\
\hline CellTrace Blue cell proliferation kit & ThermoFisher & C34568 \\
\hline Recombinant Mouse IL-10 (carrier-free) & Biolegend & 575804 \\
\hline Recombinant Mouse TGF- $\beta 1$ (carrier-free) & Biolegend & 763104 \\
\hline \multicolumn{3}{|l|}{ Critical commercial assays } \\
\hline Agilent RNA 6000 Pico Kit & Agilent & $5067-1513$ \\
\hline SMARTseq v4 Ultra-low input kit & Clontech & 634888 \\
\hline RNeasy micro kit & Qiagen & 74004 \\
\hline Colloidal Blue Staining Kit & ThermoFisher & LC6025 \\
\hline Direct-zol 96 kit & Zymo Research & $\mathrm{R} 2054$ \\
\hline Quant-iT RiboGreen RNA Assay kit & ThermoFisher & R11490 \\
\hline Pan Human T Cell Isolation Kit & Miltenyi & $130-096-535$ \\
\hline MojoSort Mouse CD4 T cell Isolation Kit & BioLegend & 480033 \\
\hline Dynabeads Protein $G$ & ThermoFisher & $10003 \mathrm{D}$ \\
\hline
\end{tabular}




\begin{tabular}{|c|c|c|}
\hline \multicolumn{3}{|l|}{ Oligonucleotides } \\
\hline Actb & ThermoFisher & $\underline{\mathrm{Mm} 02619580 \_\mathrm{g} 1}$ \\
\hline Rps10 & ThermoFisher & Mm02391992_g1 \\
\hline Rpl14 & ThermoFisher & Mm00782569_s1 \\
\hline Dhx34 & ThermoFisher & Mm01268294_g1 \\
\hline eIF3e & ThermoFisher & Mm01700222_g1 \\
\hline Rpl8 & ThermoFisher & Mm00657299 g1 \\
\hline \multicolumn{3}{|l|}{ Deposited data } \\
\hline Analysis Code & This paper & $\begin{array}{l}\text { https://github.com/B } \\
\text { enaroyaResearch/P2 } \\
\text { 32RiboSeq }\end{array}$ \\
\hline Raw Data & This Paper & $\begin{array}{l}\text { GEO } \\
\text { ID:GSE171789 }\end{array}$ \\
\hline \multicolumn{3}{|l|}{ Experimental models: organisms/strains } \\
\hline B6J.129(Cg)-Rpl22 ${ }^{\text {tml.lPsam }} / \mathrm{SjJ}$ & Jackson Laboratories & 02997 \\
\hline B6.Cg-Tg(Cd4-cre)1Cwi/BfluJ & Jackson Laboratories & 022071 \\
\hline B6.C-Tg(CMV-cre $) 1 \mathrm{Cgn} / \mathrm{J}$ & Jackson Laboratories & 006054 \\
\hline B6.129(Cg)-Foxp $3^{\operatorname{tm} 3(D T R / G F P) A y r} / \mathrm{J}$ & Jackson Laboratories & 016958 \\
\hline B6.129(Cg)-Foxp $3^{\text {tm } 4(Y F P / i c r e) A y r} / \mathrm{J}$ & Jackson Laboratories & 016959 \\
\hline B6.SJL-Ptprc ${ }^{a} P e p c^{b} /$ BoyJ & Jackson Laboratories & 002014 \\
\hline IL-10RB-/- (B6.129S2-I110rbtm1Agt/J & Jackson Laboratories & 005027 \\
\hline \multicolumn{3}{|l|}{ Software and algorithms } \\
\hline STAR 2.4.2a read aligner & Dobin et al. 2013 & $\begin{array}{l}\text { https://github.com/al } \\
\text { exdobin/STAR }\end{array}$ \\
\hline LIMMA & Ritchie et al. 2015 & $\begin{array}{l}\text { https://www.biocond } \\
\text { uctor.org/packages/r } \\
\text { elease/bioc/html/lim } \\
\text { ma.html }\end{array}$ \\
\hline fgsea & $\begin{array}{l}\text { Korotkevich et al. } \\
2019\end{array}$ & $\begin{array}{l}\text { https://www.biorxiv. } \\
\text { org/content/10.1101/ } \\
060012 \mathrm{v} 3\end{array}$ \\
\hline Htseq-count & Anders et al. 2014 & $\begin{array}{l}\text { https://github.com/ht } \\
\text { seq/htseq }\end{array}$ \\
\hline
\end{tabular}

